\title{
THE KO-VALUED SPECTRAL FLOW FOR SKEW-ADJOINT FREDHOLM OPERATORS
}

\author{
CHRIS BOURNE, ALAN L. CAREY, MATTHIAS LESCH, AND ADAM RENNIE
}

This article is dedicated to Krzysztof Wojciechowski, our friend and colleague whom we have missed for over a decade. His interest and contributions to index theory and geometry have been a constant source of inspiration.

\begin{abstract}
Aвstract. In this article we give a comprehensive treatment of a 'Clifford module flow' along paths in the skew-adjoint Fredholm operators on a real Hilbert space that takes values in $\mathrm{KO}_{*}(\mathbb{R})$ via the Clifford index of Atiyah-Bott-Shapiro. We develop its properties for both bounded and unbounded skew-adjoint operators including an axiomatic characterization. Our constructions and approach are motivated by the principle that

spectral flow $=$ Fredholm index.

That is, we show how the KO-valued spectral flow relates to a $\mathrm{KO}$-valued index by proving a Robbin-Salamon type result. The Kasparov product is also used to establish a spectral flow $=$ Fredholm index result at the level of bivariant $\mathrm{K}$ theory. We explain how our results incorporate previous applications of $\mathbb{Z} / 2 \mathbb{Z}-$ valued spectral flow in the study of topological phases of matter.
\end{abstract}

\section{CONTENTS}

Introduction

1. Motivation from physical theory 4

2. Clifford algebras and the ABS construction 6

3. Some useful homotopies and the Cayley transform $\quad 15$

4. Fredholm pairs and the Clifford index 18

5. The $\mathrm{KO}$-valued spectral flow 25

6. Extension to unbounded operators 30

7. Uniqueness of the $\mathrm{KO}$-valued spectral flow 35

Date: 2020-03-31.

2010 Mathematics Subject Classification. 19K56, 46L80, 81 T75.

Key words and phrases. Fredholm index, spectral flow, Clifford algebra, K- and KK-theory.

C.B. is supported by a JSPS Grant-in-Aid for Early-Career Scientists (No. 19K14548).

A.L.C. acknowledges the financial support of the Australian Research Council.

M.L. would like to thank the following institutions: The School of Mathematics and Applied Statistics of the University of Wollongong and the University of Sydney for their hospitality, the international visitor program of the University of Sydney for financial support, the University of Bonn for granting a sabbatical semester and the Hausdorff Center for Mathematics, Bonn, for financial support.

C.B., A.L.C. and A.R. thank the Erwin Schrödinger Institute program Bivariant K-Theory in Geometry and Physics for hospitality and support during the production of this work. 
8. The Robbin-Salamon Theorem for KO-valued spectral flow 37

9. Spectral flow and the Kasparov product 39

Appendix A. Some classical homotopy equivalences 42

References $\quad 45$

\section{INTRODUCTION}

In this paper we examine the theory and applications of spectral flow for operators on a real Hilbert space. Aspects of the theory have been in place since Atiyah and Singer [AtSi69]. Recent developments in the mathematical study of topological phases necessitates a more systematic approach.

Background. The first occurrence of the notion of spectral flow for loops in the space of skew-adjoint Fredholm operators on a real Hilbert space appears in an article of Witten [WiT82]. From a more mathematical perspective the first study is due to Lott [Lот88] who, drawing inspiration from Witten's ideas, introduced the notion of 'Clifford module-valued spectral flow' for loops in the classifying spaces of real K-theory. He followed the treatment by Atiyah-Singer for these classifying spaces [ATSI69]. These early papers also closely followed the article of Atiyah, Patodi, and Singer [APS76, Sec. 7] by introducing spectral flow using the notion of 'intersection number'.

The present article is partly motivated by recent applications of the notion of 'real spectral flow' in the study of topological phases of matter [CPSB19, DSBW19] where skew-adjoint Fredholm operators arise naturally from Hamiltonians in quantum systems. Our focus, however, is to give a comprehensive treatment of the mathematical questions that these applications have raised. We nevertheless sketch how notions of spectral flow that have previously appeared in the study of topological phases relate to our results.

Our first observation is that the intersection number definition of spectral flow is not helpful for the applications to topological phases that have emerged recently. For these, an analytic approach is needed, particularly for aperiodic and disordered systems whose spectra cannot be easily studied in general. For spectral flow along paths of self-adjoint Fredholm operators on a complex Hilbert space, an analytic approach began in [PHI96] and was extended in [BBLPo5]. This analytic approach may be adapted to the case of spectral flow along paths in the skew-adjoint Fredholm operators on a real Hilbert space. One method of achieving this is found in [CPSB19] where a $\mathbb{Z} / 2 \mathbb{Z}$-valued spectral flow in the skew-adjoint Fredholm operators on a real Hilbert space was defined.

The study of topological phases can be related to the classifying spaces of KO-theory [ALZı97, Kiтo9]. Here we exploit the construction by Atiyah-Singer [AtSi69, KAR7o, ScH93] of these spaces in terms of skew-adjoint Fredholm operators and develop in this framework a general analytic theory of spectral flow. 
The main results. Our first objective is the generalization of the analytic $\mathbb{Z} / 2 \mathbb{Z}$ valued spectral flow of [CPSB19] along paths in the space of skew-adjoint Fredholm operators on a real Hilbert space to a 'Clifford module flow' that takes values in $\mathrm{KO}_{*}(\mathbb{R})$ via the Clifford index of Atiyah-Bott-Shapiro [ABS64]. After setting out the preliminaries in the early sections, we give the definition in Sec. 5 and its relation to all previously studied spectral flow. We develop its properties for both bounded and unbounded skew-adjoint operators and present an axiomatic framework for this $\mathrm{KO}$-valued spectral flow that is analogous to that already existing in the complex case in [LESO5].

Our constructions and approach are motivated by the principle that

$$
\text { spectral flow }=\text { Fredholm index. }
$$

That is, we show how $\mathrm{KO}$-valued spectral flow relates to a $\mathrm{KO}$-valued index by proving in Theorem 8.1 in Sec. 8 a Robbin-Salamon type result [RoSA95]. Kasparov's bivariant theory has also come to play a role in applications to models of topological phases of matter. With this in mind we extend Theorem 8.1 to show how the Kasparov product may be used to establish a spectral flow = Fredholm index result at the level of bivariant K-theory. The proof is a straightforward application of the constructive Kasparov product [KALE13].

Organization of the paper. After a brief introduction to the physical motivations underpinning our work in Sec. 1, Sec. 2 gives a careful introduction to Clifford algebras and to the ABS Clifford index [ABS64]. As it will be useful for applications we go into some explicit details. Then Sec. 3 expands on [ATSI69] and contains preliminaries needed for later proofs.

The essentially new material starts in Sec. 4 where we introduce a KO-valued index for Fredholm pairs of complex structures on a real Hilbert space. This is the real counterpart to the long-standing concept of Fredholm pairs of projections. There is of course a link between them which we explain. We note that this section is directly relevant to the applications to topological phases.

The KO-valued spectral flow for paths of bounded skew-adjoint Fredholm operators is introduced next in Sec. 5. We collect its fundamental properties and show that it incorporates previous examples of real spectral flow that have been discussed in the literature. The extension to the unbounded case then follows in Sec. 6.

Our KO-valued spectral flow can be characterized uniquely in an axiomatic fashion as we explain in Sec. 7. This characterization enables us to give a short proof of our Robbin-Salamon type theorem in Sec. 8. Finally, in Sec. 9 we relate this theorem to Kasparov theory by exploiting the unbounded Kasparov product. The latter viewpoint is relevant for applications to topological phases and the bulk-edge correspondence. We collect some homotopy results from [AтSi69, MrL63] that will be of use to us in Appendix A. 


\section{Motivation FROM PHYSICAL THEORY}

As we remarked earlier, motivation for this paper comes from issues raised by the mathematical aspects of studies of topological phases for fermionic one particle systems. Historically model Hamiltonians of these fermion systems commute or anti-commute with prescribed symmetry operators that may arise as unitary or anti-unitary involutive operators on a complex Hilbert space. Such anti-unitary symmetries then determine a real structure on the complex space and one may consider topological phase labels of Hamiltonians that respect given symmetries and real structure. Our results in this paper show that these topological phase labels are identified by $\mathrm{KO}-$ valued spectral flow.

Previous work, notably [KITo9, FrMo13] and further developed in (amongst other places) [THI16] showed that the symmetries of the Hamiltonians that allow topological phases generate real Clifford algebras. This leads on to the idea of detecting different topological phases using the Clifford module valued index of Atiyah-Bott-Shapiro. Some of the present authors showed that one could apply Kasparov's bivariant theory in the real case to realize these Clifford module indices [BCR16, BKR17].

The connection of topological phases with real index theory of skew-adjoint Fredholm operators arises in its simplest form as follows. There is a class of model Hamiltonians that are said to be of Bogoliubov-de Gennes type (these are called symmetry class D in the physics literature). That is, there is a complex Hilbert space $\mathrm{H}_{\mathbb{C}}$ equipped with a particle-hole symmetry given by an anti-linear involution $\mathrm{C}$ on $\mathrm{H}_{\mathbb{C}}$. The symmetry operator $\mathrm{C}$ is self-adjoint, $\mathrm{C}^{2}=1$ and $\mathrm{CHC}=$ $-\mathrm{H}$ with $\mathrm{H}=\mathbf{H}^{*}$ the (bounded) self-adjoint Hamiltonian of the system. Then the operator $\mathrm{iH}$ commutes with $\mathrm{C}$ and gives a skew-adjoint operator on the real Hilbert space $\mathrm{H}_{\mathbb{R}}=\left\{v \in \mathrm{H}_{\mathbb{C}}: \mathrm{C} v=v\right\}$. It is normally assumed in these models that $0 \notin \sigma(\mathbf{H})$ and so $i \mathbf{H}$ is an invertible (and therefore Fredholm) skew-adjoint operator on a real Hilbert space.

The space of all skew-adjoint Fredholm operators commuting with $\mathrm{C}$ has nontrivial topology. One method of detecting this topology is to consider paths of such Hamiltonians $\left\{\boldsymbol{H}_{t}\right\}_{t \in[0,1]}$ that respect the particle hole symmetry $\mathrm{CH}_{\mathrm{t}} \mathrm{C}=-\mathbf{H}_{\mathrm{t}}$ for all $t \in[0,1]$. If we restrict to Fredholm paths, where $\operatorname{ker}\left(\mathbf{H}_{t}\right)$ is finite dimensional for all $t$, we obtain a path of skew-adjoint Fredholm operators $\left\{i H_{t}\right\}_{t \in[0,1]}$ on the real Hilbert space $H_{\mathbb{R}}$. If we also assume the endpoints $\mathbf{H}_{0}$ and $\mathbf{H}_{1}$ are invertible (e.g. we consider Fredholm paths connecting two gapped Bogoliubov-de Gennes Hamiltonians), this path can be completed into a loop. As such, topological properties of Hamiltonians in symmetry class D are closely connected to the loop space of skew-adjoint Fredholm operators, as was studied in detail in [CPSB19]. Such loops give us the simplest example of $\mathrm{KO}$-valued spectral flow and was termed $\mathbb{Z} / 2 \mathbb{Z}$ spectral flow.

It was observed in [BoSB2o] that the $\mathbb{Z} / 2 \mathbb{Z}$-valued spectral flow provides an alternative method to describe the relative topological phase of Bogoliubov-de Gennes Hamiltonians without any other information (e.g. dimension or a Brillouin torus). Because the topological phase of gapped free-fermion Hamiltonians 
may take values in any of the groups $\mathrm{KO}_{*}(\mathbb{R})$, there is a clear motivation to fully develop the notion of a general $\mathrm{KO}$-valued spectral flow that incorporates systems with symmetries. A related point of view may also be found in [DSBW19].

To consider paths of Hamiltonians with symmetries and a generalized spectral flow, the picture of free-fermionic topological phases developed by Kennedy and Zirnbauer [KeZi16] and more recently Alldridge, Max and Zirnbauer [AMZ19] is of particular use to us. Following this description, we again consider a self-adjoint Bogoliubov-de Gennes Hamiltonian $\mathbf{H}, \mathbf{C H C}=-\mathbf{H}$, such that $\mathbf{H}$ commutes with prescribed physical symmetry operators [ALZ197, HHZo5]. A key observation described in detail in [KeZI16] is that these physical symmetry operators can be used to construct a representation of a real Clifford algebra acting on the real Hilbert space $\mathrm{H}_{\mathbb{R}}$ of elements fixed by $\mathrm{C}$. Furthermore, the generators of this Clifford representation anti-commute with the real skew-adjoint operator $\mathrm{iH}$ and its 'spectral flattening' $J_{\mathbf{H}}=\mathbf{i} \mathbf{H}|\mathbf{H}|^{-1}$. That is, free-fermionic gapped Hamiltonians with Altland-Zirnbauer-type symmetries are in one-to-one correspondence with skew-adjoint gapped operators on a real Hilbert space that anti-commute with the generators of a Clifford algebra representation. Representations of all ten Morita classes of Clifford algebras can be constructed by considering the different Altland-Zirnbauer symmetry types. Topological phases have been associated to such systems by classical homotopy methods or van Daele K-theory [KeZi16, AMZ19]. See also [Kel17, Kel19].

Given a fixed Altland-Zirnbauer symmetry type, we can consider paths of Hamiltonians $\left\{\boldsymbol{H}_{t}\right\}_{t \in[0,1]}$ that respect the given symmetry for all $t \in[0,1]$. Passing to the real subspace fixed under the anti-linear involution, the path $\left\{\mathrm{iH}_{\mathrm{t}}\right\}_{\mathrm{t} \in[0,1]}$ will anti-commute with the Clifford generators that come from the symmetry type for all $t$. By restricting to Fredholm paths with invertible endpoints, we therefore obtain a loop of skew-adjoint Fredholm operators on a real Hilbert space that anti-commute with a fixed number of Clifford generators. By constructing a KO-valued spectral flow that defines a homomorphism of the loop group of such skew-adjoint Fredholm operators onto an Atiyah-Bott-Shapiro index group, we obtain a mathematically precise formulation of the physical description of topological phases as homotopy classes of symmetric Hamiltonians. That is, the KO-valued spectral flow precisely encodes the topological obstruction for two symmetric and gapped free-fermion Hamiltonian systems to be connected by a continuous Fredholm path that respects the underlying symmetries. Conversely, a non-trivial $\mathrm{KO}$-valued spectral flow guarantees the existence of topological zero energy states (i.e. a kernel) at some point along the path joining two gapped Hamiltonians.

Let us also note that the application of spectral flow to systems and models in condensed matter physics has a rich history. The connection between charge transport, Chern numbers and the Hall conductance with the index of a pair of projections (used heavily in Phillip's definition [PHI96] of spectral flow) was studied in detail by Avron, Seiler and Simon [ASS94A, ASS94B]. The index of a pair of projections continues to provide a useful mechanism to characterize 
bulk topological phases in the physics literature, see [LiMo19] for example. In the mathematical physics literature, the index of skew-adjoint Fredholm operators and generalizations of the index of a pair of projections have been used to characterize the strong topological phase in all dimensions and symmetry classes [GrSB16, KaKo18].

Recently Schulz-Baldes and co-authors have used spectral flow constructions to study the strong invariant of topological insulators (potentially with anti-linear symmetries) in two-dimensional systems [DNSB16] and in complex classes of topological phases (symmetry type A and AIII) in arbitrary dimension [CASB19]. Such spectral flows are usually implemented physically via a flux or monopole insertion through a local cell in a lattice system. Our work more closely follows [CPSB19, DSBW19], which concerns the homotopy type of Hamiltonians and Fredholm operators with certain symmetries and without reference to a Brillouin zone or pairing with a Dirac-type element in K-homology, some basic examples are given in Sec. 5.3. Therefore, while our KO-valued spectral flow covers all symmetry classes of free-fermionic topological phases, it is in general an additional step to relate this spectral flow to a strong or weak topological phase.

While our results in the current paper shed new light on $\mathrm{KO}_{\mathrm{r}}$-valued spectral flow for all $r$, the instance that is completely unexplored previously is that of $\mathrm{KO}_{4}$. Because our constructions do not make reference to lattice dimension, $\mathrm{KO}_{4}-$ valued spectral flow corresponds to Hamiltonians in symmetry class AII. In this case the kernels of the skew-adjoint Fredholm operators are quaternionic Hilbert spaces. Thus we obtain from our results the notion of quaternionic spectral flow. One may write down explicit Hamiltonians which realize this case though the construction is lengthy and we will not attempt it here. These model Hamiltonians are however of the general form that we describe later in Sec. 5.3.

\section{CliffFord algebras and the ABS construction}

Since it will be important for us we briefly recall here the ABS construction, cf. [ABS64, LAMi89]. We put an emphasis, though, on the real Clifford algebras $\mathrm{Cl}_{r, s}$ with $\mathrm{r}$ self-adjoint and $s$ skew-adjoint generators. The extension of the ABS construction to this case, cf. [ATI66, KAR70], is straightforward, but as far as we know, not easily accessible in published form.

2.1. Preliminaries and notation. The purpose of this section is to fix some terminology and notation. It will be in effect through the whole paper. This section should be consulted first in case the reader is looking for the definition of a certain notation.

By $\mathbb{Z}, \mathbb{R}, \mathbb{C}$ we denote the sets of integers, real numbers, and complex numbers resp.

Let $\mathrm{C}_{r, s}$ denote the (real) Clifford algebra on $r+s$ unitary generators $e_{1}, \ldots, e_{r}$, $f_{1}, \ldots, f_{s}$ subject to the relations

$$
\begin{aligned}
& e_{i} e_{j}+e_{j} e_{i}=2 \delta_{i j}, \quad f_{k} f_{l}+f_{k} f_{l}=-2 \delta_{k l}, \quad e_{i} f_{k}+f_{k} e_{i}=0, \\
& f_{j}^{*}=-f_{j}, \quad e_{j}^{*}=e_{j}, \quad \text { for } 1 \leq i, j \leq r, \quad 1 \leq k, l \leq s .
\end{aligned}
$$


The algebra $C \ell_{r, s}$ is a $\mathbb{Z} / 2 \mathbb{Z}$-graded algebra by declaring the generators to be of odd parity. The product of all generators

$$
\omega_{r, s}:=e_{1} \cdot \ldots \cdot e_{r} \cdot f_{1} \cdot \ldots \cdot f_{s}, \quad \omega_{r, s}^{2}= \begin{cases}(-1)^{r}, & r+s \equiv 3 \text { or } 4(\bmod 4), \\ (-1)^{r+1}, & r+s \equiv 1 \text { or } 2(\bmod 4),\end{cases}
$$

is called the volume element. It is central if $r+s$ is odd and it implements the grading if $r+s$ is even.

Definition 2.1 (Clifford element, Clifford generator). Elements $e_{1}, \ldots, e_{r}, f_{1}, \ldots, f_{s}$ in an algebra (e.g. on a Hilbert space) satisfying the relations (2.1) will be addressed as Clifford elements or, if the generated algebra is emphasized, Clifford generators.

Our convention for labeling $\mathrm{C}_{r, s}$ is the same as in [KAs8o, AMZ19] and opposite to [LAMi89]. Occasionally, for illustration purposes we translate results to the complex case, where $\mathbb{C} \ell_{r, s}=\mathbb{C} \ell_{0, r+s}=\mathbb{C} \ell_{r+s, 0}=: \mathbb{C} \ell_{r+s}$ denotes the complex Clifford algebra on $r+s$ generators (same relations).

Definition $2.2\left(\mathrm{C}_{\mathrm{r}, \mathrm{s}}\right.$-Hilbert space, Clifford module). A real resp. complex Hilbert space is a vector space $\mathrm{H}$ over the $\mathbb{R}$ resp. $\mathbb{C}$ together with an inner product $\langle\cdot, \cdot\rangle\rangle$ such that $\mathrm{H}$ together with the norm $\|x\|:=\sqrt{\langle x, x\rangle}$ is complete.

Let $\mathrm{H}$ be a separable real Hilbert space and let $\mathcal{B}(\mathrm{H})$ be the $\mathrm{C}^{*}$-algebra of bounded linear operators on $\mathrm{H}$. We call $\mathrm{H}$ a Cl, $\mathrm{Cl}_{r, s}$-Hilbert space if $\mathrm{C}_{r, s}$ is represented as a $\mathrm{C}^{*}$-algebra on $\mathrm{H}$. In this case, the generators are then denoted by $\mathrm{E}_{1}, \ldots, \mathrm{E}_{\mathrm{r}}, \mathrm{F}_{1}, \ldots, \mathrm{F}_{\mathrm{s}} \in \mathcal{B}(\mathrm{H})$.

A finite dimensional $\mathrm{C}_{\mathrm{r}, \mathrm{s}}$-Hilbert space will also be called a Clifford module, still with the understanding that it is equipped with an inner product such that the $\mathrm{Cl}_{r, s^{-}}$ action is a $\mathrm{C}^{*}$-algebra representation.

For a $\mathrm{C}_{r, s}-$ Hilbert space we denote by

$$
\mathcal{B}^{r, s+1}(H):=\left\{\mathrm{T} \in \mathcal{B}(H) \mid T=-T^{*}, \begin{array}{l}
E_{j} T=-T_{j}, j=1, \ldots, r, \\
F_{k} T=-T_{k}, k=1, \ldots, s
\end{array}\right\}
$$

the skew-adjoint operators which are $\mathrm{C}_{\mathrm{r}, \mathrm{s}}$-anti-linear. In particular, $\mathcal{B}^{0,1}(\mathrm{H})$ denotes the skew-adjoint bounded operators.

Occasionally, the corner case $r=s=0$ comes up. A C $l_{0,0}-$ Hilbert space is just a real Hilbert space with the understanding that $E_{0}=I$. Furthermore, we put $\mathcal{B}^{0,0}(\mathrm{H})=\mathcal{B}(\mathrm{H})$ (this corresponds to $s=-1$ ).

For real and complex Hilbert spaces a bounded linear operator $u$ is called 'unitary' if $u^{*} u=u u^{*}=I$, that is in the real case we use 'orthogonal' and 'unitary' as synonyms.

A $\mathrm{Cl}_{r, s}$-Hilbert space will be called standard if the multiplicity of each irreducible representation of $\mathrm{Cl}_{r, s}$ on $\mathrm{H}$ is infinite.

For separable Hilbert spaces the $\infty$ multiplicity assumption is not really serious. Firstly, if $r-s \not \equiv 1(\bmod 4)$ it is a non-issue as $C \ell_{r, s}$ has only 1 irreducible representation ([LAMi89, Theorem 5.7], beware the different convention). If $r-s \equiv 1(\bmod 4)$ then there are 2 irreducible representations. Given a concrete $\mathrm{H}$ with a $\mathrm{Cl}_{r, s}$ action we can always find another separable Hilbert space $\mathrm{H}^{\prime}$ 
such that $\mathrm{H} \oplus \mathrm{H}^{\prime}$ satisfies the $\infty$ multiplicity assumption and hence is a standard $\mathrm{Cl}_{\mathrm{r}, \mathrm{s}}-$ Hilbert space.

We add the remark that if the $\mathrm{C}_{r, s}-$ Hilbert space is standard then it can be written as $\mathrm{H}=\mathrm{H}^{\prime} \oplus \mathrm{H}^{\prime}$, where the decomposition is $\mathrm{C}_{r, s}$-linear and $\mathrm{H}^{\prime}$ itself is standard. Putting $E_{r+1}:=\left(\begin{array}{ll}0 & I \\ I & 0\end{array}\right), F_{s+1}:=\left(\begin{array}{cc}0 & -I \\ I & 0\end{array}\right), H$ is equipped with a standard $\mathrm{Cl}_{\mathrm{r}+1, \mathrm{~s}+1}$ structure. Inductively, this can be continued and hence on a standard $C \ell_{r, s}$-Hilbert space we may always assume that the infinite Clifford algebra $\bigcup_{r, s} \mathrm{Cl}_{r, s}$ is acting and that for each $r, s$ the action of $\mathrm{Cl}_{r, s}$ is standard. In particular, we may always assume that there are as many Clifford generators $E_{j}, F_{k}$ at our disposal as we please.

All standard $\mathrm{Cl}_{r, s}$-Hilbert spaces are equivalent as $\mathrm{Cl}_{\mathrm{r}, \mathrm{s}}-$ modules and there is the following obvious Absorption Theorem: given a $\mathrm{Cl}_{r, s}-$ Hilbert space $\mathrm{H}$ and a standard $\mathrm{Cl}_{r, s}-$ Hilbert space $\mathrm{H}^{\prime}$ then $\mathrm{H} \oplus \mathrm{H}^{\prime}$ is a standard $\mathrm{Cl}_{r, s}-$ Hilbert space and hence equivalent to $\mathrm{H}^{\prime}$ as a $\mathrm{Cl}_{r, s}$-module.

Finally, $\mathcal{K}(\mathrm{H})$ denotes the $\mathrm{C}^{*}$-algebra of compact operators and by $\pi: \mathcal{B}(\mathrm{H}) \rightarrow$ $Q(H)=\mathcal{B}(H) / \mathcal{K}(H)$ we denote the quotient map onto the Calkin algebra. For an operator $T \in \mathcal{B}(H)$ we will use the abbreviation $\|T\|_{\mathcal{Q}(H)}:=\|\pi(T)\|_{\mathcal{Q}(H)}$ for the norm of $\pi(T)=T+\mathcal{K}(\mathrm{H})$ in the Calkin algebra. If there is only one Hilbert space around, the disambiguator " $(\mathrm{H})$ " will occasionally be omitted.

We will also have to deal with finite dimensional Clifford modules, where homotopy issues are a priori a bit more delicate. We will, however always be able to specialize to a stable situation, see Appendix A.2.

2.2. Isomorphisms of Clifford algebras. For later reference, we introduce the $2 \times 2$ matrices

$$
\begin{aligned}
\mathrm{K}_{1} & :=\left(\begin{array}{cc}
1 & 0 \\
0 & -1
\end{array}\right), \quad \mathrm{K}_{2}:=\left(\begin{array}{ll}
0 & 1 \\
1 & 0
\end{array}\right), \quad \mathrm{L}_{1}:=\left(\begin{array}{cc}
0 & -1 \\
1 & 0
\end{array}\right), \\
\omega_{1,1} & =\mathrm{K}_{1} \cdot \mathrm{L}_{1}=-\mathrm{K}_{2}, \quad \omega_{2,0}=\mathrm{K}_{1} \cdot \mathrm{K}_{2}=-\mathrm{L}_{1}, \quad \mathrm{~K}_{1} \cdot \mathrm{K}_{2} \cdot \mathrm{L}_{1}=\mathrm{I}_{2} .
\end{aligned}
$$

Note that, see e.g. [LAMr89, Sec. I.4], $\mathrm{Cl}_{1,0}=\mathrm{C}^{*}\left(\mathrm{~K}_{1}\right) \simeq \mathbb{R} \oplus \mathbb{R}, \mathrm{Cl}_{2,0}=$ $\mathrm{C}^{*}\left(\mathrm{~K}_{1}, \mathrm{~K}_{2}\right)=\mathrm{C}^{*}\left(\mathrm{~K}_{1}, \mathrm{~L}_{1}\right)=\mathrm{C}_{1,1}=\mathrm{M}(2, \mathbb{R}){ }^{1}$ and $\mathrm{C} \ell_{0,2} \simeq \mathrm{C}^{*}\left(\mathrm{~K}_{1} \otimes \mathrm{L}_{1}, \mathrm{~K}_{2} \otimes \mathrm{L}_{1}\right) \simeq \mathbb{H}$, the latter being the quaternions.

From now on we will view $\mathbb{R}^{2}$ as a $C \ell_{2,1}$-module with generators $K_{1}, K_{2}, L_{1}$, as a $C \ell_{1,1}$-module with generators $\mathrm{K}_{1}, \mathrm{~L}_{1}$ as well as a $C \ell_{0,1}$ resp. $C \ell_{1,0}$-module with generator $L_{1}$ resp. $K_{1}$.

We note further that $C^{*}\left(K_{1}, K_{2}, L_{1}\right)=M(2, \mathbb{R})$ is one of the two irreducible representations of $\mathrm{Cl}_{2,1} \simeq \mathrm{M}(2, \mathbb{R}) \oplus \mathrm{M}(2, \mathbb{R})$. The representation is characterized by the fact that the (representation of the) volume element $\omega_{2,1}:=\mathrm{K}_{1} \cdot \mathrm{K}_{2} \cdot \mathrm{L}_{1}=\mathrm{I}_{2}$ is the $2 \times 2$ unit matrix.

Our choice of matrices $K_{1}$ and $K_{2}$ is of course arbitrary and other choices are possible. For example, we can exchange $K_{1}$ and $K_{2}$ by conjugating by the unitary $\frac{1}{\sqrt{2}}\left(K_{1}+K_{2}\right)$. Though we note that this transformation will reverse the orientation,

\footnotetext{
${ }^{1}$ For a ring $\mathrm{R}$ we denote by $\mathrm{M}(\mathrm{k}, \mathrm{R})$ the $\mathrm{k} \times \mathrm{k}$-matrices over $\mathrm{R}$.
} 
where we recall $\omega_{2,0}=K_{1} K_{2}$ and similarly will send $L_{1} \mapsto-L_{1}$. Hence such transformations can introduce a global minus sign and should be used with care.

Proposition 2.3. 1. Recall [LAMi89, Sec. I.4] the following isomorphisms of Clifford algebras:

$$
\begin{aligned}
& C \ell_{r+1, s+1} \simeq C \ell_{r, s} \otimes C \ell_{1,1} \simeq M\left(2, C \ell_{r, s}\right) \simeq \begin{cases}M\left(2^{r+1}, C \ell_{0, s-r}\right), & \text { if } s \geq r, \\
M\left(2^{s+1}, C \ell_{r-s, 0}\right), & \text { if } r \geq s,\end{cases} \\
& \mathrm{Cl}_{\mathrm{r}+2, \mathrm{~s}} \simeq \mathrm{Cl}_{\mathrm{s}, \mathrm{r}} \otimes \mathrm{Cl}_{2,0}, \\
& \mathrm{C}_{\mathrm{r}, \mathrm{s}+2} \simeq \mathrm{C} \ell_{\mathrm{s}, \mathrm{r}} \otimes \mathrm{Cl}_{0,2}, \\
& \mathrm{C} \ell_{r+1, s} \simeq \mathrm{C}_{s+1, r} .
\end{aligned}
$$

2.a $A \mathrm{Cl}_{\mathrm{r}+1, \mathrm{~s}+1}$-Hilbert space is $\mathrm{C}_{\mathrm{r}+1, \mathrm{~s}+1}$-covariantly isomorphic to $\mathrm{H}=\mathrm{H}^{\prime} \otimes \mathbb{R}^{2}$ with $\mathrm{H}^{\prime}$ being a $\mathrm{Cl}_{\mathrm{r}, \mathrm{s}}$-Hilbert space and such that $\mathrm{E}_{\mathrm{r}+1}=\mathrm{I}_{\mathrm{H}^{\prime}} \otimes \mathrm{K}_{1}$ and $\mathrm{F}_{\mathrm{s}+1}=\mathrm{I}_{\mathrm{H}^{\prime}} \otimes \mathrm{L}_{1}$.

2.b Similarly, a $\mathrm{C} \ell_{\mathrm{r}+2, \mathrm{~s}}$-Hilbert space is $\mathrm{C}_{\mathrm{r}+2, \mathrm{~s}}-$ covariantly isomorphic to $\mathrm{H}=\mathrm{H}^{\prime} \otimes$ $\mathbb{R}^{2}$ with $\mathrm{H}^{\prime}$ being a $\mathrm{Cl}_{\mathrm{s}, \mathrm{r}}$-Hilbert space and such that $\mathrm{E}_{\mathrm{r}+\mathrm{j}}=\mathrm{I}_{\mathrm{H}^{\prime}} \otimes \mathrm{K}_{\mathrm{j}}, \mathrm{j}=1,2$.

2.c Finally, a standard $C \ell_{1,0}$-Hilbert space is covariantly isomorphic to $\mathrm{H}^{\prime} \otimes \mathbb{R}^{2}$ with $\mathrm{E}_{1}=\mathrm{I}_{\mathrm{H}^{\prime}} \otimes \mathrm{K}_{1}$.

The decompositions in 2.a-2.c will be used frequently below. The tensor products in Eq. (2.6)-(2.8) are ordinary tensor products of graded algebras ${ }^{2}$ and the isomorphisms respect the natural grading. For the next section it will be crucial that the isomorphism Eq. (2.9) does not respect the grading.

Proof. Let us first comment on the decompositions 2.a-2.c. In 2.c the standard assumption guarantees that the eigenspaces of $E_{1}$ are infinite-dimensional and hence isomorphic.

2.a/2.b The first two cases may be treated in parallel by putting $G=F_{s+1}$ in the first case and $G:=E_{r+2}$ in the second. Since $E_{r+1}$ and $G$ anti-commute, $G$ interchanges the two \pm 1 -eigenspaces of $E_{r+1}$. Let $H^{\prime}:=\operatorname{ker}\left(E_{r+1}-I\right)$ be the +1 eigenspace of $E_{r+1}$ and put

$$
\Psi: \mathrm{H}^{\prime} \otimes \mathbb{R}^{2} \longrightarrow \mathrm{H}, \quad\left(\begin{array}{l}
x \\
y
\end{array}\right) \mapsto x+\mathrm{Gy} .
$$

Then one checks that

$$
\Psi^{-1} E_{r+1} \Psi=I_{H^{\prime}} \otimes K_{1}, \quad \Psi^{-1} G \Psi= \begin{cases}I_{H^{\prime}} \otimes K_{2}, & G=E_{r+2}, \\ I_{H^{\prime}} \otimes L_{1}, & G=F_{s+2},\end{cases}
$$

and for any other Clifford element $X \in\left\{E_{1}, \ldots, E_{r}, F_{1}, \ldots, F_{s}\right\}$

$$
\Psi^{-1} X \Psi=\left\{\begin{array}{lll}
X E_{r+2} \otimes \omega_{2,0}, & G=E_{r+2}, & \omega_{2,0}=K_{1} \cdot K_{2}=-L_{1}, \\
F_{s+1} X \otimes \omega_{1,1}, & G=F_{s+1}, & \omega_{1,1}=K_{1} \cdot L_{1}=-K_{2} .
\end{array}\right.
$$

${ }^{2}$ This statement might be confusing: if $A$ and $B$ are $\mathbb{Z} / 2 \mathbb{Z}$-graded algebras their tensor product $A \otimes B$ is graded by putting $\operatorname{deg}(a \otimes b):=\operatorname{deg} a+\operatorname{deg} b(\bmod 2)$ for homogeneous elements $a \in$ $A, b \in B$. This is to be distinguished from the graded (skew-commutative) tensor product $A \hat{\otimes} B$. The algebra $A \hat{\otimes} B$ has the same underlying vector space $A \otimes B$ and the same grading, but the product is $a \hat{\otimes} b \cdot a^{\prime} \hat{\otimes} b^{\prime}:=(-1)^{\operatorname{deg} b \operatorname{deg} a^{\prime}} a a^{\prime} \hat{\otimes} b b^{\prime}$. See also [KAs8o, Sec. 2.6]. 
Thus, letting $C \ell_{r, s}$ (resp. $C \ell_{s, r}$ ) act on $H^{\prime}$ with generators $F_{s+1} X\left(\right.$ resp. $X E_{r+2}$ ), $X \in\left\{E_{1}, \ldots, E_{r}, F_{1}, \ldots, F_{s}\right\}, \Psi^{-1}$ is the isomorphism we are looking for. Note, that $\left(X E_{r+2}\right)^{2}=-X^{2}$ and $\left(X F_{s+1}\right)^{2}=X^{2}$, thus $X E_{r+2}, X \in\left\{E_{1}, \ldots, E_{r}, F_{1}, \ldots, F_{s}\right\}$ satisfy the $\mathrm{C}_{s, \mathrm{r}}-$ relations.

1. The previous consideration immediately leads to the proof of Eq. (2.6) and Eq. (2.7). Namely, the isomorphism $\mathrm{C}_{r+1, s+1} \rightarrow \mathrm{C}_{r, s} \otimes \mathrm{C}_{1,1}$ is given by sending $\left\{e_{1}, \ldots, e_{r}, f_{1}, \ldots, f_{s}\right\} \ni X \mapsto X \otimes \omega_{1,1}$ and $f_{s+1} \mapsto 1 \otimes L_{1}, e_{r+1} \mapsto 1 \otimes K_{1}$. Note that $\omega_{1,1}^{2}=\mathrm{I}_{2}$.

Similarly, the isomorphism $\mathrm{C}_{r+2, s} \rightarrow C \ell_{s, r} \otimes C \ell_{2,0}$ is given by sending $e_{j} \mapsto$ $f_{j}^{\prime} \otimes \omega_{2,0}, j=1, \ldots, r, f_{k} \mapsto e_{k}^{\prime} \otimes \omega_{2,0}, k=1, \ldots, s$, and $e_{r+2} \mapsto 1 \otimes K_{2}, e_{r+1} \mapsto 1 \otimes K_{1}$. Here, $e_{k}^{\prime}, f_{j}^{\prime}$ denote the generators of $C \ell_{s, r}$. Note that $\omega_{2,0}^{2}=-I_{2}$.

Analogously, the isomorphism $\mathrm{C}_{r, s+2} \rightarrow \mathrm{C}_{\mathrm{s}, \mathrm{r}} \otimes \mathrm{Cl}_{0,2}$ is given by sending $e_{j} \mapsto$ $f_{j}^{\prime} \otimes \omega_{0,2}, j=1, \ldots, r, f_{k} \mapsto e_{k}^{\prime} \otimes \omega_{0,2}, k=1, \ldots, s$, and $f_{s+1} \mapsto 1 \otimes L_{1}, f_{s+2} \mapsto 1 \otimes L_{2}$, where $\mathrm{L}_{1}, \mathrm{~L}_{2}$ are the generators of $\mathrm{Cl}_{0,2}$. Note that $\omega_{0,2}^{2}=-\mathrm{I}_{2}$.

Finally, the isomorphism $\mathrm{Cl}_{\mathrm{r}+1, s} \rightarrow \mathrm{C}_{s+1, r}$ is given as follows: inside the algebra $C \ell_{r+1, s}$ consider the elements $e_{1}^{\prime}:=f_{1} \cdot e_{r+1}, \ldots, e_{s}^{\prime}:=f_{s} \cdot e_{r+1}, e_{s+1}^{\prime}:=e_{r+1}$, $f_{1}^{\prime}:=e_{1} \cdot e_{r+1}, \ldots, f_{r}^{\prime}:=e_{r} \cdot e_{r+1} \cdot e_{1}^{\prime}, \ldots, f_{r}^{\prime}$ satisfy the Clifford relations for $C \ell_{s+1, r}$. Hence the map $C \ell_{s+1, r} \rightarrow C \ell_{r+1, s}, e_{j} \mapsto e_{j}^{\prime}, f_{k} \mapsto f_{k}^{\prime}$ is the (inverse of) the claimed isomorphism. This also shows that the isomorphism does not respect the grading.

Remark 2.4. 1. We note in passing a consequence of the isomorphism Eq. (2.9). Given a $C \ell_{r+1, s}-$ Hilbert space with generators $E_{1}, \ldots, E_{r+1}, F_{1}, \ldots, F_{s}$, it becomes a $C \ell_{s+1, r}$-Hilbert space with generators $E_{j}^{\prime}=F_{j} \cdot E_{r+1}, j=1, \ldots, s, E_{s+1}^{\prime}=E_{r+1}$, $\mathrm{F}_{\mathrm{k}}^{\prime}=\mathrm{E}_{\mathrm{k}} \cdot \mathrm{E}_{\mathrm{r}+1}, \mathrm{k}=1, \ldots, \mathrm{r}$. Furthermore, given a $\mathrm{C} \ell_{\mathrm{r}+1, \mathrm{~s}}$-anti-linear operator $\mathrm{T} \in \mathcal{B}(\mathrm{H})$ with $\mathrm{T}^{*}=\varepsilon \mathrm{T}, \varepsilon \in\{ \pm 1\}$, then $\widetilde{\mathrm{T}}=\mathrm{TE}_{\mathrm{r}+1}$ is $\mathrm{Cl}_{\mathrm{s}+1, \mathrm{r}}$-anti-linear with respect to the generators $E_{1}^{\prime}, \ldots, F_{r}^{\prime}$ and $\widetilde{T}^{*}=-\varepsilon \widetilde{T}$. Clearly, if $T$ is Fredholm then so is $\widetilde{T}$.

Thus, there is a parallel theory of $\mathrm{Cl}_{r, s}$-anti-linear self-adjoint operators. In light of the isomorphism Eq. (2.9) the translation is straightforward. See, however, the Remark 2.10 below.

2. For the decompositions 2.a-2.c we note that for $T \in \mathcal{B}(H)$ with the notation introduced at the beginning of Sec. 2.2,

$$
\begin{aligned}
\mathrm{T}_{1,1} & :=\left(\begin{array}{cc}
0 & -\mathrm{T}^{*} \\
\mathrm{~T} & 0
\end{array}\right)=\mathfrak{R}(\mathrm{T}) \otimes \mathrm{L}_{1}+\Im(\mathrm{T}) \otimes \mathrm{K}_{2}, \\
\mathfrak{R}(\mathrm{T}) & :=\frac{1}{2}\left(\mathrm{~T}+\mathrm{T}^{*}\right), \quad \mathfrak{I}(\mathrm{T}):=\frac{1}{2}\left(\mathrm{~T}-\mathrm{T}^{*}\right) .
\end{aligned}
$$

The operator $T_{1,1}$ is skew-adjoint and anti-commutes with $K_{1}$. If $T$ is already skewadjoint, then $\mathfrak{R}(T)=0$ and $T_{1,1}$ anti-commutes with $L_{1}$ as well. Furthermore, if $T$ already has $\mathrm{Cl}_{r, s}-\mathrm{Clifford}$ symmetries then under the isomorphism Eq. (2.6) the operator $\mathrm{T}_{1,1}$ has $\mathrm{C}_{\mathrm{r}+1, s+1}$-Clifford symmetries.

2.3. The ABS construction. Denote by $\widehat{M}_{r, s}$ resp. $\mathcal{M}_{r, s}$ the Grothendieck group of graded resp. ungraded finite dimensional $\mathrm{C}_{r, s}-$ modules (with inner product) 
with addition being direct sum. For $s \geq 1$ we have $\widehat{\mathcal{M}}_{r, s} \simeq \mathcal{M}_{r, s-1}$ by sending the graded $\mathrm{Cl}_{r, s}$-module $\mathrm{V}$ to the ungraded $\mathrm{Cl}_{r, s-1} \simeq \mathrm{C}_{r, s}^{0}$ module $\mathrm{V}^{0} .{ }^{3}$ Here, $C \ell_{r, s}^{0}$ is identified with $C \ell_{r, s-1}$ by sending $e_{i} \cdot f_{s}$ to $e_{i}, i=1, \ldots, r$ and $f_{i} \cdot f_{s}$ to $f_{i}$, $i=1, \ldots, s-1$.

Also, cf. the second part of Prop. 2.3 above, given a graded $\mathrm{C}_{r, s}-$ module $\mathrm{V}$ with grading operator $\varepsilon, V$ becomes an ungraded $C \ell_{r+1, s}$-module by sending $e_{r+1}$ to $\varepsilon$. Thus $\hat{\mathcal{M}}_{\mathrm{r}, \mathrm{s}} \simeq \mathcal{M}_{\mathrm{r}+1, \mathrm{~s}}$ for all s. Altogether, we have $\mathcal{M}_{\mathrm{r}, \mathrm{s}-1} \simeq \mathcal{M}_{\mathrm{r}+1, \mathrm{~s}}$ and the isomorphism is canonical by sending the $C \ell_{r, s-1}$-module $V$ to $V \otimes \mathbb{R}^{2}$ with $C \ell_{r+1, s+1} \simeq C \ell_{r, s} \otimes C \ell_{1,1}$ action given by $X \otimes \omega_{1,1}, I_{V} \otimes K_{1}, I_{V} \otimes L_{1}$.

Furthermore, let

$$
\begin{aligned}
& A_{r, s}:=\widehat{\mathcal{M}}_{r, s} / \widehat{\mathcal{M}}_{r, s+1}\left(\simeq \mathcal{M}_{r, s-1} / \mathcal{M}_{r, s} \quad \text { if } s \geq 1\right), \\
& \mathrm{B}_{r, s}:=\widehat{\mathcal{M}}_{r, s} / \hat{\mathcal{M}}_{r+1, s}\left(\simeq \mathcal{M}_{r, s-1} / \mathcal{M}_{r+1, s-1} \quad \text { if } s \geq 1\right) .
\end{aligned}
$$

One of the main results of [ABS64], [LAMr89, Sec. I.9] is that $A_{0, k}$ is canonically isomorphic to $\mathrm{KO}_{\mathrm{k}}(\mathbb{R})$.

The isomorphisms Eq. (2.6)-Eq. (2.9) and the 8-periodicity for the Clifford algebras immediately imply that $A_{r, s}, B_{r, s}$ depend only on the difference $s-r(\bmod 8)$. Furthermore, the ungraded isomorphism Eq. (2.9) shows for all $r, s \geq 1$

$$
\mathrm{B}_{\mathrm{r}, \mathrm{s}} \simeq \mathcal{M}_{\mathrm{r}, \mathrm{s}-1} / \mathcal{M}_{\mathrm{r}+1, s-1} \simeq \mathcal{M}_{s, r-1} / \mathcal{M}_{s, r} \simeq \mathrm{A}_{s, r} \text {. }
$$

A posteriori, by periodicity, this isomorphism holds for all $r, s$.

The representation theory of the Clifford algebras easily gives the following table directly:

\begin{tabular}{|c|c|c|c|c|c|c|c|c|c|}
\hline $\mathrm{s}-\mathrm{r}(\bmod 8)$ & 0 & 1 & 2 & 3 & 4 & 5 & 6 & 7 & 8 \\
$\mathrm{~A}_{\mathrm{r}, \mathrm{s}} \simeq \mathrm{B}_{\mathrm{s}, \mathrm{r}}$ & $\mathbb{Z}$ & $\mathbb{Z} / 2 \mathbb{Z}$ & $\mathbb{Z} / 2 \mathbb{Z}$ & 0 & $\mathbb{Z}$ & 0 & 0 & 0 & $\mathbb{Z}$ \\
\hline
\end{tabular}

Definition 2.5 (ABS map). Below we will explicitly describe natural isomorphisms

$$
\tau_{r, s}: A_{r, s} \longrightarrow \begin{cases}\mathbb{Z}, & s-r \equiv 0(\bmod 4), \\ \mathbb{Z} / 2 \mathbb{Z}, & s-r \equiv 1 \text { or } 2(\bmod 8) .\end{cases}
$$

The map $\tau_{\mathrm{r}, \mathrm{s}}$ will be called the ABS map.

The isomorphisms of the table are unique only up to sign (relevant in the cases $\equiv 0(\bmod 4))$, hence the choice of Clifford generators matters in concrete cases. We therefore quickly list here the concrete maps.

\subsection{Examples of the ABS map for certain $r, s$.}

2.4.1. $r=s=0$. This case can only be dealt with in the graded setting: if $V$ is a graded real vector space then $\tau_{0,0}: A_{0,0} \ni[V] \mapsto \operatorname{dim}_{\mathbb{R}} V^{0}-\operatorname{dim}_{\mathbb{R}} V^{1} \in \mathbb{Z}$. A generator of the cyclic group $A_{0,0}$ is given by the class of the graded vector space $\mathrm{V}=\mathrm{V}^{0} \oplus \mathrm{V}^{1}=\mathbb{R} \oplus 0$.

\footnotetext{
${ }^{3}$ The superscript ${ }^{0}$ refers to the even subspace w.r.t. the grading.
} 
2.4.2. $r=s \geq 1$. Using $r=s \geq 1$ allows us to deal with the case $s-r=0$ in the ungraded setting, where the definition would have us deal with representations of ' $\mathrm{C} l_{0,-1}$ '. We have

$$
\begin{aligned}
\mathrm{C} \ell_{r, r-1} & \simeq \mathrm{M}\left(2^{r-1}, C \ell_{1,0}=\mathbb{R} \oplus \mathbb{R}\right), \\
\mathrm{C} \ell_{r, r} & \simeq \mathrm{M}\left(2^{r-1}, \mathrm{C} \ell_{1,1}=\mathrm{M}(2, \mathbb{R})\right) \simeq \mathrm{M}\left(2^{r-1}, \mathrm{C} \ell_{2,0}\right) \simeq \mathrm{C} \ell_{r+1, r-1} .
\end{aligned}
$$

We recall that $A_{r, r}=\mathcal{M}_{r, r-1} / \mathcal{M}_{r, r} \simeq B_{r, r}=\mathcal{M}_{r, r-1} / \mathcal{M}_{r+1, r-1}$ and since $C l_{r, r}=$ $\mathrm{Cl}_{\mathrm{r}+1, \mathrm{r}-1}=\mathrm{M}\left(2^{\mathrm{r}}, \mathbb{R}\right)$ the two quotients are just equal. Let $\mathrm{V}$ be a finite dimensional $\mathrm{Cl}_{r, r-1}-$ module.

Note that (cf. [LAMi89, Theorem 5.7], Sec. 3) $\mathrm{C}_{r, r-1}$ has two irreducible representations. The volume element $\omega_{r, r-1}=e_{1} \cdot \ldots \cdot e_{r} \cdot f_{1} \cdot \ldots \cdot f_{r-1}$ is central and satisfies $\omega_{r, r-1}^{*}=\omega_{r, r-1}, \omega_{r, r-1}^{2}=I$. The two irreducible representations $\rho_{ \pm}$ of $\mathrm{C}_{r, r-1}$ are $2^{r-1}$-dimensional and they are characterized by $\rho_{ \pm}\left(\omega_{r, r-1}\right)= \pm \mathrm{I}$. Hence their multiplicities in $V$ are given by $2^{1-r} \cdot \operatorname{dim}_{\mathbb{R}} \operatorname{ker}\left(\omega_{r, r-1} \mp I\right)$. If $V$ carries a $C \ell_{r+1, r-1} \simeq C \ell_{r, r}$ structure, then since $\omega_{r, r-1}$ must anti-commute with the additional Clifford generator, the two multiplicities must coincide. Conversely, if these two multiplicities coincide, $E_{r+1}$ resp. $F_{r}$ may be constructed.

Thus the natural identification of $A_{r, r}=B_{r, r}$ with $\mathbb{Z}$ is given by

$$
\tau_{r, r}: A_{r, r} \ni[V] \mapsto \frac{1}{2^{r-1}} \cdot\left(\operatorname{dim}_{\mathbb{R}} \operatorname{ker}\left(\omega_{r, r-1}-I\right)-\operatorname{dim}_{\mathbb{R}} \operatorname{ker}\left(\omega_{r, r-1}+I\right)\right) \in \mathbb{Z}
$$

E.g., a positive generator of the cyclic group $A_{1,1}$ is given by the $C \ell_{1,0}$ module $\left(\mathbb{R}, \mathrm{E}_{1}=\mathrm{I}\right)$.

In general, a positive generator of the cyclic group $A_{r, r}$ is given by $\mathbb{R}^{2^{r-1}}$ together with a choice of Clifford matrices $E_{1}, \ldots, E_{r}, F_{1}, \ldots, F_{r-1}$ such that $E_{1} \cdot \ldots \cdot E_{r} \cdot F_{1}$. $\ldots \cdot \mathrm{F}_{\mathrm{r}-1}=\mathrm{I}$.

Since $C \ell_{r, r+7} \simeq M\left(16, C \ell_{r, r-1}\right)$, up to some tweaking with matrix algebras, the previous analysis carries over to all $s \geq r \geq 1$ with $s-r \equiv 0(\bmod 8)$. Details are left to the reader.

2.4.3. $s=r+1$. A finite dimensional ungraded real $C \ell_{r, r}=M\left(2^{r}, \mathbb{R}\right)$-module $V$ carries a $C \ell_{r, r+1} \simeq \mathrm{M}\left(2^{\mathrm{r}}, \mathrm{C} \ell_{0,1} \simeq \mathbb{C}\right)$-module structure if and only if $\mathrm{V}$ decomposes into an even number of irreducible $\mathrm{C} \ell_{r, r}$-modules. Thus

$$
\tau_{r, r+1}: A_{r, r+1} \ni[V] \mapsto \frac{1}{2^{r}} \operatorname{dim}_{\mathbb{R}} V(\bmod 2) \in \mathbb{Z} / 2 \mathbb{Z}
$$

A generator of the cyclic group $A_{r, r+1}$ is given by the (up to equivalence unique) $2^{r}$-dimensional $\mathrm{Cl}_{r, r}$-module, i.e. $\mathbb{R}^{2^{r}}$ with a choice of Clifford matrices $E_{1}, \ldots, E_{r}$, $F_{1}, \ldots, F_{r}$. Given such a module, there is a unique choice of another Clifford generator $E_{r+1}$ such that $E_{1} \cdot \ldots \cdot E_{r+1} \cdot F_{1} \cdot \ldots \cdot F_{r}=I$. Thus, the module may at the same time be viewed as a generator of $A_{r+1, r+1}$.

2.4.4. $s=r+2$. Let $\left(V, F_{1}\right)$ be a finite dimensional ungraded $C l_{0,1}$-module. Then $F_{1}$ is a complex structure and there exists $F_{2} \in \mathcal{B}(V)$ with $F_{2}^{*}=-F_{2}, F_{1} F_{2}=-F_{2} F_{1}$ if and only if $\operatorname{dim}_{\mathbb{C}} V=\frac{1}{2} \operatorname{dim}_{\mathbb{R}} V \equiv 0(\bmod 2)$. Thus the map of the table is given 
by $A_{0,2} \ni[V] \mapsto \operatorname{dim}_{\mathbb{C}}\left(V, i=F_{1}\right)(\bmod 2)=\frac{1}{2} \operatorname{dim}_{\mathbb{R}} V(\bmod 2)$. A generator of the cyclic group $A_{0,2}$ is given by $\left(\mathbb{R}^{2}, L_{1}=\left(\begin{array}{cc}0 & -1 \\ 1 & 0\end{array}\right)\right)$.

For arbitrary $r$, cf. the previous discussion, the map is given by

$$
\tau_{r, r+2}: A_{r, r+2} \ni[V] \mapsto \frac{1}{2^{r+1}} \operatorname{dim}_{\mathbb{R}} V(\bmod 2) \in \mathbb{Z} / 2 \mathbb{Z} .
$$

2.4.5. $s=r+4, c f$. the case $r=s$ above. This case is mostly parallel to Sec. $2.4 \cdot 2$ above as $C \ell_{r, r+3} \simeq \mathrm{M}\left(2^{r}, \mathbb{H} \oplus \mathbb{H}\right)$ has two $2^{r+2}$-dimensional irreducible representations $\rho_{ \pm}$which are distinguished by $\rho_{ \pm}\left(\omega_{r, r+3}\right)= \pm I$. Hence their multiplicities in a $C \ell_{r, r+3}-$ module $V$ are given by $2^{-r-2} \cdot \operatorname{dim}_{\mathbb{R}} \operatorname{ker}\left(\omega_{r, r+3} \mp \mathrm{I}\right)$. Again, $V$ carries a $\mathrm{Cl}_{r, r+4}$ structure if and only if these multiplicities coincide. In sum, the natural identification of $A_{r, r+4}$ with $\mathbb{Z}$ is given by

$$
\tau_{r, r+4}: A_{r, r+4} \ni[V] \mapsto \frac{1}{2^{r+2}}\left(\operatorname{dim}_{\mathbb{R}} \operatorname{ker}\left(\omega_{r, r+3}-I\right)-\operatorname{dim}_{\mathbb{R}} \operatorname{ker}\left(\omega_{r, r+3}+I\right)\right) \in \mathbb{Z}
$$

In applications to topological phases, where spaces are typically taken to be complex, the above quaternionic index is often computed with complex dimension and takes range in $2 \mathbb{Z}$, see [GRSB16, KaKo18] for example.

We note an important consequence of the previous discussion.

Theorem 2.6. There are natural forgetful maps $\mathrm{fg}_{\mathrm{r}+1, \mathrm{~s}}: A_{\mathrm{r}+1, \mathrm{~s}} \rightarrow A_{\mathrm{r}, \mathrm{s}}$ sending the $\mathrm{C}_{\mathrm{r}+1, \mathrm{~s}-1}$-module $\mathrm{V}$ to the underlying $\mathrm{C} \ell_{r, s-1}-$ module.

1. For $\mathrm{fg}_{2,2}$ we have $\tau_{1,2}\left(\mathrm{fg}_{2,2}([\mathrm{~V}])\right)=\tau_{2,2}([\mathrm{~V}])(\bmod 2)$, hence $\mathrm{fg}_{2,2}$ is surjective.

2. Furthermore, $\mathrm{fg}_{1,2}$ is an isomorphism. More concretely,

$$
\tau_{0,2}\left(\mathrm{fg}_{1,2}([\mathrm{~V}])\right)=\tau_{1,2}([\mathrm{~V}]) .
$$

Explicitly, the module $\left(\mathbb{R}^{2}, \mathrm{~K}_{1}, \mathrm{~K}_{2}, \mathrm{~L}_{1}\right)$ generates $\mathrm{KO}_{j}, \mathrm{j}=0,1,2$ in the following incarnations:

(i) $\tau_{2,2}$ of the $\mathrm{Cl}_{2,1}$-module $\left(\mathbb{R}^{2}, \mathrm{~K}_{1}, \mathrm{~K}_{2}, \mathrm{~L}_{1}\right)$ equals $1 \in \mathbb{Z}$.

(ii) $\mathrm{fg}_{2,2}\left(\mathbb{R}^{2}, \mathrm{~K}_{1}, \mathrm{~K}_{2}, \mathrm{~L}_{1}\right)=\left(\mathbb{R}^{2}, \mathrm{~K}_{1}, \mathrm{~L}_{1}\right)$ which generates $\mathrm{A}_{1,2}$ and hence

$$
\tau_{1,2}\left(\mathbb{R}^{2}, \mathrm{~K}_{1}, \mathrm{~L}_{1}\right)=1 \in \mathbb{Z} / 2 \mathbb{Z} \text {. }
$$

(iii) Finally, $\mathrm{fg}_{1,2}\left(\mathbb{R}^{2}, \mathrm{~K}_{1}, \mathrm{~L}_{1}\right)=\left(\mathbb{R}^{2}, \mathrm{~L}_{1}\right)$ which in turn generates $\mathrm{A}_{0,2}$ as

$$
\tau_{0,2}\left(\mathbb{R}^{2}, \mathrm{~L}_{1}\right)=1 \in \mathbb{Z} / 2 \mathbb{Z} \text {. }
$$

Proof. The proof follows by walking through the cases 2.4.2-2.4.4 above.

\subsection{The $\mathrm{C} \ell_{r, s}$ Fredholm index.}

Definition 2.7. Let $\mathrm{H}$ be a $\mathrm{Cl}_{r, s}-$ Hilbert space. Following Atiyah and Singer4 and recalling the notation $\mathcal{B}^{r, s+1}$ from Eq. (2.3), let

$$
\mathcal{F}^{\mathrm{r}, \mathrm{s}+1}(\mathrm{H}):=\left\{\mathrm{T} \in \mathcal{B}^{\mathrm{r}, \mathrm{s}+1}(\mathrm{H}) \mid \mathrm{T} \text { Fredholm }\right\} .
$$

\footnotetext{
${ }^{4}$ Cf. also [KAR7o]. The $\mathcal{F}^{k}$ in [AtSi69] is our $\mathcal{F}^{0, k}$. The adaption of [ATSi69] to the two parameter case is straightforward as, up to a canonical homeomorphism, $\mathcal{B}^{r, s+1}, \mathcal{F}^{r, s+1}$ depend only on the difference $s-r(\bmod 8)$.
} 
Furthermore, if $\mathrm{H}$ is standard then let $\mathcal{F}_{*}^{\mathrm{r}, \mathrm{s}+1}(\mathrm{H})=\mathcal{F}^{\mathrm{r}, \mathrm{s}+1}$ if $\mathrm{r}-\mathrm{s} \not \equiv 2(\bmod 4)$ and let $\mathcal{F}_{*}^{r, s+1}(\mathrm{H})$ be the interesting path component of $\mathcal{F}^{\mathrm{r}, \mathrm{s}+1}$ if $\mathrm{r}-\mathrm{s} \equiv 2(\bmod 4)$, see [ATSi69, p. 7]. ${ }^{5}$ Moreover, denote by $\mathrm{FO}_{*}^{\mathrm{r}, s+1} \subset \mathcal{F}_{*}^{\mathrm{r}, s+1}\left(\right.$ resp. $\left.\mathrm{FO}^{\mathrm{r}, \mathrm{s}+1} \subset \mathcal{F}^{\mathrm{r}, \mathrm{s}+1}\right)$ the space of those $\mathrm{T}$ with $\|\mathrm{T}\|=1$ and whose image $\pi(\mathrm{T}) \in \mathcal{Q}(\mathrm{H}):=\mathcal{B}(\mathrm{H}) / \mathcal{K}(\mathrm{H})$ in the Calkin algebra is unitary.

Remark 2.8. The subspace $\mathrm{FO}^{\mathrm{r}, \mathrm{s}+1}\left(\mathrm{FO}_{*}^{\mathrm{r}, s+1}\right)$ is a deformation retract of $\mathcal{F}^{\mathrm{r}, \mathrm{s}+1}$ $\left(\mathcal{F}_{*}^{r, s+1}\right)$, in particular the inclusion $\mathrm{FO}^{r, s+1} \subset \mathcal{F}^{r, s+1}\left(\mathrm{FO}_{*}^{r, s+1} \subset \mathcal{F}_{*}^{r, s+1}\right)$ is a homotopy equivalence.

For $\mathrm{T} \in \mathcal{F}^{\mathrm{r}, \mathrm{s}+1}$ the kernel ker $\mathrm{T}$ is naturally an ungraded $\mathrm{C} \mathrm{l}_{\mathrm{r}, \mathrm{s}}-$ module and one therefore puts

$$
\operatorname{ind}_{r, s+1}(T)=[\operatorname{ker} T] \in A_{r, s+1}, \quad \text { and } \operatorname{Ind}_{r, s+1}(T):=\tau_{r, s+1}\left(\operatorname{ind}_{r, s+1}(T)\right),
$$

cf. Eq. (2.17). The index ind $_{r, s+1}$ is locally constant on $\mathcal{F}^{\mathrm{r}, s+1}$, cf. [LAMr89, Sec. III.10, Prop. 10.6]. See also [GRSB16, KAKo18] for a similar construction.

We note that ind $\mathrm{r}_{\mathrm{r}, \mathrm{s}+1}$ is surjective. The following construction is instructive and very explicit. Fix a standard $C \ell_{r, s+1}-$ Hilbert space $\mathrm{H}_{0}$. Then, given a $C \ell_{r, s}-$ module $\mathrm{V}$, put $\mathrm{H}=\mathrm{H}_{0} \oplus \mathrm{V}$ and $\mathrm{T}_{\mathrm{V}}:=\left.\left.\mathrm{F}_{\mathrm{s}+1}\right|_{\mathrm{V}} \oplus 0\right|_{\mathrm{V}}$. Then

$$
\operatorname{ind}_{r, s+1}\left(T_{V}\right)=[V] \in A_{r, s+1} \simeq \mathcal{M}_{r, s} / \mathcal{M}_{r, s+1} \text {. }
$$

Clearly, the only purpose of $\mathrm{H}_{0}$ is to make $\mathrm{H}$ itself standard. Otherwise, one could just put $\mathrm{T}_{\mathrm{V}}=0$ on $\mathrm{H}=\mathrm{V}$ which has index $[\mathrm{V}]$ as well.

Theorem 2.6 has an immediate consequence for Fredholm operators.

Proposition 2.9. For a Fredholm operator $T \in \mathcal{F}^{r, r}$ one has, see Eq. (2.13), the index formula

$$
\operatorname{Ind}_{r, r}(T)=\operatorname{Ind}_{r+1, r+1}\left(\begin{array}{cc}
0 & -T^{*} \\
T & 0
\end{array}\right)=\operatorname{Ind}_{r+1, r+1}\left(T_{1,1}\right),
$$

where $\mathrm{T}_{1,1}$, defined in (2.13), is viewed as an element of $\mathcal{F}^{\mathrm{r}+1, \mathrm{r}+1}$. This formula also holds true for $\mathrm{r}=0$, where $\mathrm{T}$ is just a Fredholm operator and $\mathrm{Ind}_{0,0}$ denotes the ordinary Fredholm index.

Next denote by $\mathrm{fg}_{\mathrm{r}+1, \mathrm{~s}+1}: \mathcal{F}^{\mathrm{r}+1, \mathrm{~s}+1} \rightarrow \mathcal{F}^{\mathrm{r}, \mathrm{s}+1}$ the natural forgetful map. For $\mathrm{r} \geq 1$ and $\mathrm{T} \in \mathcal{F}^{\mathrm{r}+1, \mathrm{r}+1}$ one has the index formula

$$
\operatorname{Ind}_{r, r+1}\left(\operatorname{fg}_{r+1, r+1}(T)\right)=\operatorname{Ind}_{r+1, r+1}(T)(\bmod 2),
$$

and for $\mathrm{T} \in \mathcal{F}^{\mathrm{r}, \mathrm{r}+1}$

$$
\operatorname{Ind}_{r-1, r+1}\left(\operatorname{fg}_{r, r+1}(T)\right)=\operatorname{Ind}_{r, r+1}(T) \text {. }
$$

${ }^{5}$ When comparing, note that in [AtSi69] $r=0, s+1=k$, hence $r-s \equiv 2(\bmod 4)$ if and only if $k \equiv 3(\bmod 4)$. 
In particular, we have for a Fredholm operator $\mathrm{T}$ with $\mathrm{T}_{1,1}=\left(\begin{array}{cc}0 & -\mathrm{T}^{*} \\ \mathrm{~T} & 0\end{array}\right)$,

$$
\begin{aligned}
\operatorname{Ind}_{0,2}\left(\begin{array}{cc}
0 & T_{1,1} \\
T_{1,1} & 0
\end{array}\right) & =\operatorname{Ind}_{1,2}\left(\begin{array}{cc}
0 & T_{1,1} \\
T_{1,1} & 0
\end{array}\right) \\
& =\operatorname{Ind}_{2,2}\left(\begin{array}{cc}
0 & T_{1,1} \\
T_{1,1} & 0
\end{array}\right)(\bmod 2) \\
& =\operatorname{Ind}_{1,1}\left(T_{1,1}\right)(\bmod 2)=\operatorname{Ind}_{0,0}(T)(\bmod 2) .
\end{aligned}
$$

Remark 2.10. As explained in Remark 2.4 there is a one to one correspondence $\mathrm{T} \leftrightarrow \mathrm{TE}_{\mathrm{r}+1}$ between $\mathrm{C} \ell_{\mathrm{r}+1, \mathrm{~s}}$-anti-linear skew-adjoint Fredholm operators and $\mathrm{C} \ell_{\mathrm{s}+1, \mathrm{r}}-$ anti-linear self-adjoint Fredholm operators. The Fredholm index of $\mathrm{T}$ lies naturally in $A_{r+1, s+1}$ and is locally constant since in a nontrivial eigenspace $\operatorname{ker}\left(T^{2}+\lambda^{2}\right)$ the phase of $\mathrm{T}$ gives an additional skew-adjoint Clifford symmetry. Consequently, for a self-adjoint $C \ell_{s+1, r}$-anti-linear operator $S\left(=T E_{r+1}\right)$ the phase of $S$ gives an additional self-adjoint Clifford symmetry. Therefore, the index of $S$ lies naturally in $B_{s+1, r+1}$, which by Eq. (2.17) is isomorphic to $A_{r+1, s+1}$.

\section{SOME USEFUl homotopies AND THE CAYLEY TRANSForm}

3.1. Spaces of complex structures. In this subsection we collect and expand on some of the homotopy theoretic results from [ATSi69].

Definition 3.1. Let $\mathcal{A}$ be a real unital $C^{*}$-algebra with Clifford elements $e_{1}, \ldots, e_{r}$, $\mathrm{f}_{1}, \ldots, \mathrm{f}_{\mathrm{s}} \in \mathcal{A}$. Denote by $\mathcal{I}^{\mathrm{r}, \mathrm{s}+1}(\mathcal{A})$ the set of complex structures ${ }^{6} \mathrm{~J} \in \mathcal{A}$ anti-commuting with the Clifford elements and by $\mathcal{J}^{0,0}(\mathcal{A})$ the space of unitaries in $\mathcal{A}$.

If $\mathrm{H}$ is a $\mathrm{Cl}_{\mathrm{r}, \mathrm{s}}-$ Hilbert space then we set $\mathrm{g}^{\mathrm{r}, \mathrm{s}+1}(\mathrm{H}):=\mathrm{g}^{\mathrm{r}, \mathrm{s}+1}(\mathcal{B}(\mathrm{H}))$.

If $\mathrm{H}$ is a $\mathrm{C} \ell_{r, s}-$ Hilbert space, $s>0$ or $r=s=0$, we put

$$
\begin{aligned}
& \bar{\Omega}_{r, s}(H):=\left\{T \in \mathcal{J}^{r, s}(H) \mid T-F_{s} \text { compact }\right\}, \\
& \widetilde{\Omega}_{r, s}(H):=\left\{T \in \mathcal{J}^{r, s}(H) \mid\left\|T-F_{s}\right\|_{\Omega}<2\right\},
\end{aligned}
$$

cf. [ATSI69, p. 19]. In the case $r=s=0$ this must be read with $F_{0}=I$.

Note that for $T \in \mathcal{J}^{r, s}(H)$ the element $\pi\left(F_{s} T\right)$ is unitary and for a unitary $u$ one has the equivalence $\|\mathrm{u}+\mathrm{I}\|<2 \Leftrightarrow 1 \notin$ spec $u$ as $|z+1| \leq 2$ for $z$ on the circle with equality only for $z=1$.

For $T \in \mathcal{J}^{r, s}(H)$ this translates into the equivalence

$$
\mathrm{T} \in \widetilde{\Omega}_{\mathrm{r}, \mathrm{s}} \Leftrightarrow\left\|\mathrm{T}-\mathrm{F}_{\mathrm{s}}\right\|_{\mathcal{Q}}<2 \Leftrightarrow\left\|\mathrm{F}_{\mathrm{s}} \mathrm{T}+\mathrm{I}\right\|_{\mathcal{Q}}<2 \Leftrightarrow 1 \notin \operatorname{spec}_{\mathrm{ess}}\left(\mathrm{F}_{\mathrm{s}} \mathrm{T}\right) .
$$

When it is necessary for disambiguation we will write

$$
\bar{\Omega}_{r, s}\left(H ; E_{1}, \ldots, E_{r}, F_{1}, \ldots, F_{s}\right)
$$

to display explicitly the generators of the Clifford module structure.

\footnotetext{
${ }^{6}$ A complex structure $\mathrm{J}$ is a unitary skew-adjoint element, in particular $\mathrm{J}^{2}=-1_{\mathcal{A}}$.
} 


\subsection{Some elementary homotopies.}

Proposition 3.2. Let $\mathcal{A}$ be a real unital $\mathrm{C}^{*}$-algebra with Clifford elements $e_{1}, \ldots, e_{\mathrm{r}}$, $f_{1}, \ldots, f_{s} \in \mathcal{A}$. Then the diagonal in

$$
\left\{\left(\mathrm{J}_{0}, \mathrm{~J}_{1}\right) \in \mathcal{J}^{\mathrm{r}, \mathrm{s}+1}(\mathcal{A}) \times \mathcal{J}^{\mathrm{r}, \mathrm{s}+1}(\mathcal{A}) \mid\left\|\mathrm{J}_{0}-\mathrm{J}_{1}\right\|<2\right\}
$$

is a strong deformation retract. The result is also valid in the corner case $\mathrm{r}=\mathrm{s}+1=0$.

Proof. The result is proved for $r, s \geq 0$, the corner case $r=s+1=0$ is left to the reader. The retraction will be given explicitly. Given $J_{0}, J_{1} \in \mathcal{J}^{r, s+1}(\mathcal{A})$ with $\| J_{0}-$ $\mathrm{J}_{1} \|<2$, put $Z:=Z\left(J_{0}, J_{1}\right)=1_{\mathcal{A}}-J_{0} J_{1}$. Then $Z$ commutes with $e_{1}, \ldots, e_{r}, f_{1}, \ldots, f_{s}$ and

$$
\mathrm{ZJ}_{1}=\mathrm{J}_{0}+\mathrm{J}_{1}=\mathrm{J}_{0} \mathrm{Z}
$$

Taking adjoints we have $\mathrm{J}_{1} \mathrm{Z}^{*}=\mathrm{Z}^{*} \mathrm{~J}_{0}$, hence

$$
Z Z^{*} J_{1}=Z^{*} J_{0} Z=J_{1} Z^{*} Z \text {. }
$$

We claim that $Z_{x}:=1_{\mathcal{A}}-x J_{0} J_{1}$ is invertible for $0 \leq x \leq 1$. Indeed

$$
Z_{x}=1_{\mathcal{A}}-x\left(J_{0}-J_{1}\right) J_{1}-x J_{1}^{2}=(1+x)\left(1_{\mathcal{A}}-\frac{x}{1+\chi}\left(J_{0}-J_{1}\right) J_{1}\right),
$$

which is invertible since

$$
\left\|\frac{x}{1+\chi}\left(\mathrm{J}_{0}-\mathrm{J}_{1}\right) \mathrm{J}_{1}\right\|<\frac{2 x}{1+x} \leq 1 .
$$

In view of this and Eq. (3.4), (3.5) the retraction is now given by

$$
\left(\mathrm{J}_{0}, \mathrm{~J}_{1}\right) \mapsto\left(\mathrm{J}_{0}, \mathrm{Z}_{\chi}\left|\mathrm{Z}_{\chi}\right|^{-1} \mathrm{~J}_{1}\left(\mathrm{Z}_{\alpha}\left|\mathrm{Z}_{\alpha}\right|^{-1}\right)^{*}\right) .
$$

Lemma 3.3. Let $\mathrm{H}$ be a $\mathrm{C} \ell_{r, s}$ Hilbert space. The neighbourhood of $\mathrm{F}_{\mathrm{s}} \in \widetilde{\Omega}_{\mathrm{r}, \mathrm{s}}(\mathrm{H})$ given by

$$
\begin{aligned}
\widetilde{\Omega}_{r, s, *}: & =\left\{\omega \in \widetilde{\Omega}_{r, s} \mid\left\|\omega-F_{s}\right\|<2\right\} \\
& =\left\{\omega \in \widetilde{\Omega}_{r, s} \mid 1 \notin \operatorname{spec}\left(F_{s} \omega\right)\right\}
\end{aligned}
$$

is contractible.

Furthermore, with the map $\Phi_{r, s+1}: \mathrm{T} \mapsto-\mathrm{F}_{\mathrm{s}} \mathrm{e}^{\pi \mathrm{TF}_{\mathrm{s}}}$ of Theorem A.4 we have for invertible $\mathrm{T} \in \mathrm{FO}^{\mathrm{r}, \mathrm{s}+1}$ the inequality $\left\|\Phi_{\mathrm{r}, \mathrm{s}+1}(\mathrm{~T})-\mathrm{F}_{\mathrm{s}}\right\|<2$; that is, $\Phi_{r, s+1}(\mathrm{~T}) \in \bar{\Omega}_{\mathrm{r}, \mathrm{s}, *}$. Here, analogously to Eq. (3.6), $\bar{\Omega}_{r, s, *}:=\left\{\omega \in \bar{\Omega}_{r, s} \mid\left\|\omega-F_{s}\right\|<2\right\}$, cf. Eq. (3.1).

Proof. The second equality in Eq. (3.6) follows analogously to the equivalence Eq. (3.3). That $\widetilde{\Omega}_{r, s, *}$ is contractible follows as in the proof of Prop. 3.2.

For invertible $\mathrm{T} \in \mathrm{FO}^{\mathrm{r}, \mathrm{s}+1}$ the operator $\mathrm{TF}_{\mathrm{s}}$ is skew, invertible and of norm 1, hence

$$
\operatorname{spec}\left(\pi_{s}\right) \subset[-i, 0) \cup(0, i]
$$

and thus by the spectral mapping theorem

$$
\operatorname{spec}\left(e^{\pi \mathrm{TF}_{s}}\right) \subset S^{1} \backslash\{1\}=\left\{z \in S^{1}|| z+1 \mid<2\right\},
$$

so consequently

$$
\|\underbrace{-F_{s} e^{\pi T F_{s}}}_{\Phi_{r, s+1}(T)}-F_{s}\|<2 .
$$


The following generalization of Theorem A.4 is crucial for the theory of Fredholm pairs and the spectral flow.

Theorem 3.4. Let $f: i \mathbb{R} \rightarrow i \mathbb{R}$ be an increasing continuous function such that

(1) $f$ is strictly increasing in a neighbourhood of 0 ,

(2) $|\mathrm{f}(\mathrm{x})| \leq 1$ for all $\mathrm{x} \in \mathfrak{i} \mathbb{R}$,

(3) $f(-x)=-f(x)=\overline{f(x)}$ for all $x \in i \mathbb{R}$.

Furthermore, let $\mathrm{H}$ be a standard $\mathrm{C}_{\mathrm{r}, \mathrm{s}+1}-$ Hilbert space. Then the map

$$
\Phi_{\mathrm{f}}: \mathcal{F}_{*}^{\mathrm{r}, \mathrm{s}+1} \rightarrow \widetilde{\Omega}_{\mathrm{r}, \mathrm{s}}, \quad \mathrm{T} \mapsto-\mathrm{F}_{\mathrm{s}} e^{\pi \mathrm{f}\left(\mathrm{TF}_{\mathrm{s}}\right)}
$$

is a homotopy equivalence. Furthermore, if $\mathrm{T} \in \mathcal{F}_{*}^{\mathrm{r}, s+1}$ is invertible then $\Phi_{\mathrm{f}}(\mathrm{T}) \in \widetilde{\Omega}_{\mathrm{r}, \mathrm{s}, *}$.

A particularly nice homotopy equivalence is given by the Cayley transform

$$
\Phi: \mathcal{F}_{*}^{r, s+1} \rightarrow \widetilde{\Omega}_{r, s}, \quad \mathrm{~T} \mapsto-\mathrm{F}_{\mathrm{s}}\left(\mathrm{I}+\mathrm{TF}_{\mathrm{s}}\right)\left(\mathrm{I}-\mathrm{TF}_{\mathrm{s}}\right)^{-1} .
$$

Remark 3.5.

1. We first clarify the last remark about the Cayley transform, i.e.

$$
e^{\pi f(x)}=: \frac{1+x}{1-x}
$$

or $^{7}$

Thus indeed

$$
f(x)=\frac{1}{\pi} \log \frac{1+x}{1-x}\left(=\frac{i}{\pi} \arctan \frac{2 x / i}{1+x^{2}}\right), \quad x \in i \mathbb{R} .
$$

$$
-F_{s} e^{\pi f\left(T_{s}\right)}=-F_{s}\left(I+T F_{s}\right)\left(I-T_{s}\right)^{-1} \text {. }
$$

2. We comment on the case $r=0, s=0$. Then $\widetilde{\Omega}_{0,0}$ is the space of unitaries $\mathrm{U}$ with $\|\mathrm{U}-\mathrm{I}\|_{\mathcal{Q}}<2$ resp. $-1 \notin \operatorname{spec}_{\mathrm{ess}}(\mathrm{U})$ and the Cayley transform is $A \mapsto$ $(I+A)(A-I)^{-1}$. In the complex case, where one considers self-adjoint instead of skew-adjoint operators, $A$ should be replaced by $i A$. This should be compared to [BBLPo5] and [KILEO4, Sec. 6.1].

Proof of Theorem 3.4. This is essentially a consequence of Theorem A.4.

We first check that $\Phi_{f}$ maps $\mathcal{F}_{*}^{r, s+1}$ into $\widetilde{\Omega}_{r, s}$. Abbreviate $g(x):=e^{\pi f(x)}$. Then $g$ maps $i \mathbb{R}$ into $S^{1}$ and $g(-x)=g(x)^{-1}=\overline{g(x)}$. Thus for $T \in \mathcal{F}_{*}^{\text {r,s+1 }}$ we have

$$
\begin{aligned}
\left(F_{s} g\left(T_{s}\right)\right)^{*} & =-\bar{g}\left(T F_{s}\right) F_{s} \\
& =-g\left(-T_{s}\right) F_{s}=-F_{s} g\left(T_{s}\right),
\end{aligned}
$$

hence $\Phi_{f}\left(T_{s}\right)$ is skew-adjoint and, since $g$ takes values in $S^{1}$, unitary. Consequently, $\Phi_{f}\left(T_{s}\right)^{2}=-I$. Clearly, since both $T$ and $F_{s}$ anti-commute with the Clifford generators except $F_{s}$ it follows that $\Phi_{f}\left(T_{s}\right) \in \mathcal{J}^{r, s}(H)$.

Finally, since $T_{s}$ is a Fredholm operator, there is a gap $[-i \varepsilon, i \varepsilon]$ in the essential spectrum. The properties (1)-(3) of $f$ imply that there is a small $\operatorname{arc}\{|\arg z|<\delta\}$ around $1 \in S^{1}$ which does not meet the essential spectrum of $e^{\pi f\left(\pi_{s}\right)}$. Therefore,

$$
\left\|-\mathrm{F}_{s} \mathrm{~g}\left(\mathrm{TF}_{\mathrm{s}}\right)-\mathrm{F}_{\mathrm{s}}\right\|_{\mathcal{Q}}=\left\|g\left(\mathrm{TF}_{\mathrm{s}}\right)+\mathrm{I}\right\|_{\mathcal{Q}}<2,
$$

proving that $\Phi_{f}$ maps into $\widetilde{\Omega}_{r, s}$ as claimed.

\footnotetext{
7The formula in parenthesis on the right of Eq. (3.9) holds only for $|x|<1$.
} 
Now define the function

$$
\Psi: i \mathbb{R} \rightarrow i \mathbb{R}, \quad x \mapsto \begin{cases}x, & |x| \leq 1, \\ x /|x|, & |x| \geq 1 .\end{cases}
$$

Then $f_{\mathfrak{u}}(x):=(1-u) f(x)+u \Psi(x), 0 \leq u \leq 1$ is a homotopy of functions satisfying (1)-(3) and consequently $\Phi_{f_{u}}$ is a homotopy between $\Phi_{f}$ and $\Phi_{\psi}$.

On the deformation retract $\mathrm{FO}_{*}^{\mathrm{r}, \mathrm{s}+1} \subset \mathcal{F}_{*}^{\mathrm{r}, s+1}$ the map $\Phi_{\Psi}$ coincides with the map $\Phi_{r, s+1}$ of Theorem A.4. Thus $\Phi_{\Psi}$ and hence $\Phi_{f}$ are homotopy equivalences as claimed.

Finally, if $\mathrm{T}$ is invertible then one argues as in the proof of Lemma 3.3. Namely, $f\left(T_{s}\right)$ is invertible as well and hence $1 \notin \operatorname{spec}\left(e^{\pi f\left(\pi_{s}\right)}\right)$, thus

$$
\left\|-F_{s} e^{\pi f\left(T_{s}\right)}-F_{s}\right\|<2 \text {. }
$$

\section{Fredholm pairs and the Clifford index}

Here we introduce a Fredholm theory of pairs of complex structures where the index naturally takes values in $A_{r, s}=\mathcal{M}_{r, s-1} / \mathcal{M}_{r, s} \simeq \mathrm{KO}_{s-r}(\mathbb{R})$, cf. Sec. 2 . The complex analogue has a long history. Kato systematically studied Fredholm pairs of subspaces in a Banach space [KAT95, IV.4.1]. The first hint that in the Hilbert space setting one should instead look at the corresponding orthogonal projections as the primary objects can be found in [BDF73, Rem. 4.9]. These projections appear prominently in the theory of boundary value problems for Dirac type operators [BBWo93, Sec. 24]. The theory was rediscovered and further developed in the influential paper [ASS94A] by Avron, Seiler, and Simon; see also [BRLEO1]. For further details see Sec. 4.2.3 and Sec. 4.2.4.

\subsection{Fredholm pairs of complex structures.}

Definition 4.1. Let $\mathrm{H}$ be a $\mathrm{Cl}_{\mathrm{r}, \mathrm{s}}$-Hilbert space. A pair $\left(\mathrm{J}_{0}, \mathrm{~J}_{1}\right)$ of elements of $\mathrm{g}^{\mathrm{r}, \mathrm{s}+1}(\mathrm{H})$ is called a Fredholm pair if $\left\|\mathrm{J}_{0}-\mathrm{J}_{1}\right\|_{Q}<2$.

Recall from Sec. 2.1 that $\|\ldots\|_{Q}$ is an abbreviation for $\|\pi(\ldots)\|_{Q}$.

Given a Fredholm pair $\left(J_{0}, J_{1}\right) \in g^{r, s+1}(H) \times g^{r, s+1}(H)$ we note that $H$ becomes a $\mathrm{C}_{r, s+1}-$ Hilbert space by setting $\mathrm{F}_{s+1}=\mathrm{J}_{0}$. Moreover, the space of those $\mathrm{J}_{1}$ with $\left(\mathrm{J}_{0}, \mathrm{~J}_{1}\right)$ being a Fredholm pair is nothing but the space

$$
\widetilde{\Omega}_{r, s+1}\left(H ; E_{1}, \ldots, E_{r}, F_{1}, \ldots, F_{s}, F_{s+1}=J_{0}\right),
$$

see Eq. (3.1).

From now on we fix a $\mathrm{C}_{r, s}-$ Hilbert space $\mathrm{H}$. The useful identities of the next Lemma are straightforward to check.

Lemma 4.2 ([CPSB19, Lemma 5.3], [ASS94A, Theorem 2.1]). Let $\mathrm{J}_{0}, \mathrm{~J}_{1} \in \mathrm{g}^{\mathrm{r}, \mathrm{s}+1}(\mathrm{H})$. Define the skew-adjoint operators

$$
\mathrm{T}_{0}=\frac{1}{2}\left(\mathrm{~J}_{0}+\mathrm{J}_{1}\right), \quad \mathrm{T}_{1}=\frac{1}{2}\left(\mathrm{~J}_{0}-\mathrm{J}_{1}\right) .
$$


Then $\mathrm{T}_{0}^{2}+\mathrm{T}_{1}^{2}=-1, \mathrm{~T}_{0} \mathrm{~T}_{1}=-\mathrm{T}_{1} \mathrm{~T}_{0}, \mathrm{~T}_{0} \mathrm{~J}_{0}=\mathrm{J}_{1} \mathrm{~T}_{0}, \mathrm{~T}_{0} \mathrm{~J}_{1}=\mathrm{J}_{0} \mathrm{~T}_{0}, \mathrm{~T}_{1} \mathrm{~J}_{0}=-\mathrm{J}_{1} \mathrm{~T}_{1}$, and $\mathrm{T}_{1} \mathrm{~J}_{1}=-\mathrm{J}_{0} \mathrm{~T}_{1}$. Furthermore, both $\mathrm{T}_{0}$ and $\mathrm{T}_{1}$ anti-commute with the Clifford generators $\mathrm{E}_{1}, \ldots, \mathrm{E}_{\mathrm{r}}$ and $\mathrm{F}_{1}, \ldots, \mathrm{F}_{\mathrm{s}}$.

Definition and Proposition 4.3 (Definition of $\left.\operatorname{ind}_{\mathrm{r}, \mathrm{s}+2}^{1}\left(\mathrm{~J}_{0}, \mathrm{~J}_{1}\right)\right)$. Let $\left(\mathrm{J}_{0}, \mathrm{~J}_{1}\right)$ be a Fredholm pair in $\mathrm{g}^{\mathrm{r}, \mathrm{s}+1}(\mathrm{H})$. Then for all $\mathrm{t} \in[0,1]$, the operator $\gamma(\mathrm{t})=(1-\mathrm{t}) \mathrm{J}_{0}+\mathrm{t} \mathrm{J}_{1} \in \mathcal{F}^{\mathrm{r}, \mathrm{s}+1}$ is a skew-adjoint Fredholm operator anti-commuting with $\mathrm{E}_{1}, \ldots, \mathrm{E}_{\mathrm{r}}, \mathrm{F}_{1}, \ldots, \mathrm{F}_{\mathrm{s}}$, and

$$
\Phi_{r, s+1}(\gamma(t)):=-F_{s} e^{\pi \gamma(t) F_{s}}, \quad 0 \leq t \leq 1
$$

is a loop in $\widetilde{\Omega}_{\mathrm{r}, \mathrm{s}}(\mathrm{H})$.

Now embed the $\mathrm{C}_{\mathrm{r}, \mathrm{s}+1}-$ Hilbert space $\left(\mathrm{H} ; \mathrm{E}_{1}, \ldots, \mathrm{E}_{\mathrm{r}}, \mathrm{F}_{1}, \ldots, \mathrm{F}_{\mathrm{s}}, \mathrm{F}_{\mathrm{s}+1}:=\mathrm{J}_{0}\right) \hookrightarrow \mathrm{H}^{\prime}$ into a standard $C \ell_{r, s+1}-$ Hilbert space and put $\mathrm{J}_{j}^{\prime}:=\left.\mathrm{J}_{j} \oplus \mathrm{F}_{\mathrm{s}+1}\right|_{\mathrm{H}^{\perp}}$. Then we define $\operatorname{ind}_{\mathrm{r}, \mathrm{s}+2}^{1}\left(\mathrm{~J}_{0}, \mathrm{~J}_{1}\right)$ to be the class of the loop $\left.\Phi_{\mathrm{r}, \mathrm{s}+1} \circ \gamma \oplus \mathrm{F}_{\mathrm{s}}\right|_{\mathrm{H}^{\perp}}=\Phi_{\mathrm{r}, \mathrm{s}+1} \circ\left(\left.\gamma \oplus \mathrm{F}_{\mathrm{s}+1}\right|_{\mathrm{H}^{\perp}}\right)$ in $\pi_{1}\left(\widetilde{\Omega}_{r, s}\left(H^{\prime}\right), F_{s}\right)$, which by Theorem A.6 is canonically isomorphic to the abelian group $\mathrm{A}_{\mathrm{r}, \mathrm{s}+2}=\mathcal{M}_{\mathrm{r}, \mathrm{s}+1} / \mathcal{M}_{\mathrm{r}, \mathrm{s}+2} \simeq \mathrm{KO}_{\mathrm{s}+2-\mathrm{r}}(\mathbb{R})$.

Alternatively and equivalently, ind $\mathrm{r}_{\mathrm{r}, \mathrm{s}+2}^{1}\left(\mathrm{~J}_{0}, \mathrm{~J}_{1}\right)$ denotes the stable homotopy class of the

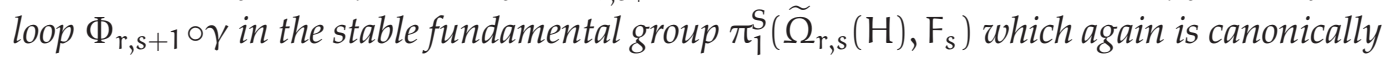
isomorphic to $A_{r, s+2}$, see Sec. A.2.

Proof. By construction $\gamma(t)$ is skew-adjoint and anti-commutes with the Clifford generators $E_{1}, \ldots, E_{r}, F_{1}, \ldots, F_{s}$. To see that it is a Fredholm operator we note that

$$
\left\|-\mathrm{J}_{1} \gamma(\mathrm{t})-\mathrm{I}\right\|_{\mathrm{Q}}=\left\|\gamma(\mathrm{t})-\mathrm{J}_{1}\right\|_{Q}<1 \text { for } \frac{1}{2} \leq \mathrm{t} \leq 1,
$$

and similarly $\left\|\mathrm{J}_{0} \gamma(\mathrm{t})-\mathrm{I}\right\|_{\mathrm{Q}}<1$ for $0 \leq \mathrm{t} \leq \frac{1}{2}$.

To prove the claim about $\Phi_{r, s+1} \circ \gamma$ we note that $\Phi_{r, s+1}(\gamma(0))=\Phi_{r, s+1}(\gamma(1))=F_{s}$. From Eq. (4.1) it follows that for all $0 \leq t \leq 1, \gamma(t) F_{s}$ lies in the open 1 ball around a unitary of square $-1\left(J_{0} F_{s}\right.$ resp. $\left.J_{1} F_{s}\right)$. Thus $\operatorname{spec}_{e s s}\left(\gamma(t) F_{s}\right) \subset[-i, 0) \cup(0, i]$ and hence $1 \notin \operatorname{spec}_{\text {ess }}\left(e^{\pi \gamma(t) F_{s}}\right)$. Consequently, cf. Eq. (3.3), $\left\|\Phi_{r, s+1}(\gamma(t))-F_{s}\right\|_{Q}<2$ and $\gamma$ is a loop in $\widetilde{\Omega}_{\mathrm{r}, \mathrm{s}}$ based at $\mathrm{F}_{\mathrm{s}}$ as claimed.

It is an immediate consequence of the construction that $\mathrm{ind}_{\mathrm{r}, \mathrm{s}+2}^{1}$ is locally constant on the space of Fredholm pairs $\left(J_{0}, J_{1}\right) \in \mathcal{J}^{r, s+1}(H) \times J^{r, s+1}(H)$.

We provide an alternative approach to the index of a Fredholm pair, whose consequence will be the important additivity formula Prop. 4.7 below.

Proposition 4.4. Let $\left(\mathrm{J}_{0}, \mathrm{~J}_{1}\right) \in \mathrm{J}^{\mathrm{r}, \mathrm{s}+1}(\mathrm{H}) \times \mathrm{g}^{\mathrm{r}, \mathrm{s}+1}(\mathrm{H})$ be a Fredholm pair and let $\mathrm{T}_{0}, \mathrm{~T}_{1}$ as in Lemma 4.2. Then

(1) The operator $\frac{1}{2}\left(\mathrm{~J}_{0}+\mathrm{J}_{1}\right)$ is Fredholm,

(2) The space $\operatorname{ker}\left(\mathrm{J}_{0}+\mathrm{J}_{1}\right)$ is an ungraded $\mathrm{C}_{r, s+1}-$ module w.r.t. the Clifford algebra generated by $\mathrm{E}_{1}, \ldots, \mathrm{E}_{\mathrm{r}}, \mathrm{F}_{1}, \ldots, \mathrm{F}_{\mathrm{s}}, \mathrm{F}_{\mathrm{s}+1}:=\mathrm{J}_{0}$.

(3) For any fixed $1>\lambda>0$, the spectral subspace $X_{\lambda}=\chi_{\left(0, \lambda^{2}\right)}\left(-T_{0}^{2}\right)$ carries naturally the structure of an ungraded $\mathrm{Cl}_{\mathrm{r}, \mathrm{s}+2}$-module with respect to the generators $\mathrm{E}_{1}, \ldots, \mathrm{E}_{\mathrm{r}}, \mathrm{F}_{1}, \ldots, \mathrm{F}_{\mathrm{s}}, \mathrm{F}_{\mathrm{r}+1}=\mathrm{J}_{0}$ and $\mathrm{F}_{\mathrm{s}+2}$ being the phase of $\mathrm{J}_{0} \mathrm{~T}_{1} \mathrm{~T}_{0}=$ $-\frac{1}{4}\left(\mathrm{~J}_{1}+\mathrm{J}_{0} \mathrm{~J}_{1} \mathrm{~J}_{0}\right), c f$. Lemma 4.2. 
Proof. We note that this is essentially [CPSB19, Lemma 5.3 and Prop. 5.2]. We will make repeated use of the identities given in Lemma 4.2.

(1). was proved in the previous proof.

(2). $\operatorname{ker}\left(\mathrm{J}_{0}+\mathrm{J}_{1}\right)$ is certainly invariant under $\mathrm{E}_{1}, \ldots, \mathrm{E}_{\mathrm{r}}, \mathrm{F}_{1}, \ldots, \mathrm{F}_{\mathrm{s}}$, and from $\mathrm{T}_{0} \mathrm{~J}_{0}=$ $\mathrm{J}_{1} \mathrm{~T}_{0}$ we infer that it is invariant under $\mathrm{J}_{0}$ as well.

(3). For part (3), we first note that $X_{\lambda}$ is $T_{1} T_{0}$-invariant, $E_{j} T_{1} T_{0}=T_{1} T_{0} E_{j}$ and $\mathrm{J}_{0} \mathrm{~T}_{1} \mathrm{~T}_{0}=-\mathrm{T}_{1} \mathrm{~T}_{0} \mathrm{~J}_{0}$.

Also observe that for $|\lambda|<1$ we have $-1<\mathrm{T}_{0}^{2} \mid \mathrm{X}_{\lambda}<0$ (the upper inequality is the Fredholm property), and so $T_{0}^{2}+T_{1}^{2}=-1$ tells us that $T_{1}$ is invertible on $X_{\lambda}$. Furthermore, $\mathrm{J}_{0} \mathrm{~T}_{1} \mathrm{~T}_{0}$ is skew-adjoint and anti-commutes with $E_{1}, \ldots, E_{r}, F_{1}, \ldots, F_{s}$, and with $\mathrm{J}_{0}$. Finally, its phase $S$ is another complex structure anti-commuting with $E_{1}, \ldots, E_{r}, F_{1}, \ldots, F_{s}, J_{0}$, and hence $X_{\lambda}$ carries the structure of a $C \ell_{r, s+2}$-module with generators $E_{1}, \ldots, E_{r}, F_{1}, \ldots, F_{s}, J_{0}, S$.

In light of this proposition we put for a Fredholm pair $\left(\mathrm{J}_{0}, \mathrm{~J}_{1}\right)$

$$
\operatorname{ind}_{r, s+2}^{2}\left(J_{0}, J_{1}\right):=\left[\operatorname{ker}\left(J_{0}+J_{1}\right)\right] \in \mathcal{M}_{r, s+1} / \mathcal{M}_{r, s+2} \text {, }
$$

i.e. the class of the $C \ell_{r, s+1}-$ module $\operatorname{ker}\left(\mathrm{J}_{0}+\mathrm{J}_{1}\right)$ in $A_{r, s+2} \simeq \mathrm{KO}_{s+2-r}(\mathbb{R})$.

It follows from (3) (cf. [CPSB19, Prop. 5.2]) that ind $\mathrm{d}_{r, s+2}^{2}$ is locally constant on the space of Fredholm pairs as well.

Theorem 4.5. The two definitions of a $\mathrm{Cl}_{\mathrm{r}, \mathrm{s}+2}$ index of a Fredholm pair $\left(\mathrm{J}_{0}, \mathrm{~J}_{1}\right)$ coincide, ind. . ind. $_{\bullet}^{2}$. Hence from now on we may just write ind $\mathrm{r}_{\mathrm{r}, \mathrm{s}+2}\left(\mathrm{~J}_{0}, \mathrm{~J}_{1}\right)$.

As for the Fredholm index we write $\operatorname{Ind}_{r, s+2}\left(J_{0}, J_{1}\right):=\tau_{r, s+2}\left(\operatorname{ind}_{r, s+2}\left(J_{0}, J_{1}\right)\right)$.

Proof. By the local constancy of both indices it suffices to check the claim for fixed $\mathrm{J}_{0} \in \mathrm{J}^{\mathrm{r}, \mathrm{s}+1}(\mathrm{H})$ on representatives of the path components of the space

$$
\left\{J \in \mathcal{J}^{r, s+1}(H) \mid\left\|J-J_{0}\right\|_{Q}<2\right\} \simeq \widetilde{\Omega}_{r, s+1}\left(H ; E_{1}, \ldots, E_{r}, F_{1}, \ldots, F_{s}, F_{s+1}=J_{0}\right)
$$

in a standard $C \ell_{r, s+1}-$ Hilbert space. By doubling the space and replacing $J_{0}$ by $\mathrm{J}_{0} \oplus-\mathrm{J}_{0}$ if necessary we may assume that the Hilbert space is standard with Jo being one of the Clifford generators. Note that both indices are not affected by stabilizing: if we add another Hilbert space $\mathrm{H}^{\prime}$ and fix $\mathrm{J}_{0}^{\prime} \in \mathrm{J}^{r, s+1}\left(\mathrm{H}^{\prime}\right)$ then $\operatorname{ind}_{r, s+2}^{\alpha}\left(J_{0}, J_{1}\right)=\operatorname{ind}_{r, s+2}^{\alpha}\left(J_{0} \oplus J_{0}^{\prime}, J_{1} \oplus J_{0}^{\prime}\right), \alpha=1,2$.

Then by Cor. A.5 we know the path components of

$$
\widetilde{\Omega}_{r, s+1}\left(H ; E_{1}, \ldots, E_{r}, F_{1}, \ldots, F_{s}, J_{0}\right)
$$

and hence we may consider $\mathrm{J}_{1}=\left.\mathrm{J}_{0}\right|_{\mathrm{H}_{0}} \oplus-\left.\mathrm{J}_{0}\right|_{\mathrm{V}}$ where $\mathrm{H}=\mathrm{H}_{0} \oplus \mathrm{V}$ is a $\mathrm{C} \ell_{r, s+1}$-linear decomposition (generators $E_{1}, \ldots, E_{r}, F_{1}, \ldots, F_{s}, F_{s+1}=J_{0}$ ) and $\operatorname{dim} V<\infty$. Then $\frac{1}{2}\left(\mathrm{~J}_{0}+\mathrm{J}_{1}\right)=\left.\left.\mathrm{J}_{0}\right|_{\mathrm{H}_{0}} \oplus 0\right|_{\mathrm{V}^{\prime}}$, whence ind $\mathrm{r}_{\mathrm{r}, \mathrm{s}+2}^{2}\left(\mathrm{~J}_{0}, \mathrm{~J}_{1}\right)=[\mathrm{V}] \in \mathcal{M}_{\mathrm{r}, \mathrm{s}+1} / \mathcal{M}_{\mathrm{r}, \mathrm{s}+2}$. Further,

$$
\gamma(\mathrm{t})=(1-\mathrm{t}) \mathrm{J}_{0}+\mathrm{t} \mathrm{J}_{1}=\left.\left.\mathrm{J}_{0}\right|_{\mathrm{H}_{0}} \oplus(1-2 \mathrm{t}) \mathrm{J}_{0}\right|_{\mathrm{V}} \cdot
$$

On $\mathrm{H}_{0}$ we have $\Phi_{\mathrm{r}, \mathrm{s}+1}(\gamma(\mathrm{t}))=-\mathrm{F}_{\mathrm{s}} \mathrm{e}^{\pi \mathrm{J}_{0} \mathrm{~F}_{\mathrm{s}}} \equiv \mathrm{F}_{\mathrm{s}}$, and on $\mathrm{V}$ we have

$$
\Phi_{r, s+1}(\gamma(t))=-F_{s} e^{\pi(1-2 t) J_{0} F_{s}}=F_{s} e^{-2 \pi t J_{0} F_{s}} .
$$


By Theorem A.6 the homotopy class of this loop is again $[\mathrm{V}]$, thus $\operatorname{ind}_{\mathrm{r}, \mathrm{s}+2}^{1}\left(\mathrm{~J}_{0}, \mathrm{~J}_{1}\right)=$ $\operatorname{ind}_{r, s+2}^{2}\left(J_{0}, J_{1}\right)$.

The proof and the known standard generators of $\mathcal{M}_{r, s+1} / \mathcal{M}_{r, s+2}$ immediately show that (cf. Sec. 2.4)

Corollary 4.6. For a standard $\mathrm{C}_{\mathrm{r}, \mathrm{s}+1}$-Hilbert space $\mathrm{H}$ the map

$$
\operatorname{ind}_{r, s+2}\left(F_{s+1}, \cdot\right): \widetilde{\Omega}_{r, s+1}(H) \rightarrow A_{r, s+2} \simeq \mathcal{M}_{r, s+1} / \mathcal{M}_{r, s+2}
$$

labels the connected components of $\widetilde{\Omega}_{r, s+1}(\mathrm{H})$. For a non-standard $\mathrm{C}_{r, s+1}-$ Hilbert space ind $_{r, s+2}$ labels the stable connected components.

Proposition 4.7. Suppose that $\left(\mathrm{J}_{0}, \mathrm{~J}_{1}\right)$ and $\left(\mathrm{J}_{1}, \mathrm{~J}_{2}\right)$ are Fredholm pairs of elements in $\mathrm{g}^{\mathrm{r}, \mathrm{s}+1}(\mathrm{H})$ with

$$
\left\|J_{0}-\mathrm{J}_{1}\right\|_{\mathcal{Q}}<1 \text { and }\left\|\mathrm{J}_{1}-\mathrm{J}_{2}\right\|_{\mathcal{Q}}<1 \text {. }
$$

Then

$$
\operatorname{ind}_{r, s+2}\left(J_{0}, J_{1}\right)+\operatorname{ind}_{r, s+2}\left(J_{1}, J_{2}\right)=\operatorname{ind}_{r, s+2}\left(J_{0}, J_{2}\right) \text {. }
$$

Proof. Denote by $\gamma_{01}, \gamma_{12}, \gamma_{02}$ the straight line paths from $J_{0}$ to $J_{1}$ etc. The assumption Eq. (4.3) implies that the straight line homotopy from $\gamma_{02}$ to the concatenation $\gamma_{12} * \gamma_{01}$ is a homotopy within paths in $\mathcal{F}^{\mathrm{r}, \mathrm{s}+1}$ since any operator $\mathrm{T}$ in the range of the homotopy satisfies $\left\|\mathrm{T}-\mathrm{J}_{0}\right\|_{Q}<2$. But then we have for the homotopy classes

$$
\left[\Phi_{r, s+1} \circ \gamma_{02}\right]=\left[\Phi_{r, s+1} \circ \gamma_{01}\right]+\left[\Phi_{r, s+1} \circ \gamma_{12}\right]
$$

in $\pi_{1}\left(\widetilde{\Omega}_{r, s}, F_{s}\right)$, thus ind $\operatorname{ins+2}_{r, s}\left(J_{0}, J_{2}\right)=\operatorname{ind}_{r, s+2}\left(J_{0}, J_{1}\right)+\operatorname{ind}_{r, s+2}\left(J_{1}, J_{2}\right)$.

4.2. Examples and comparison with the classical index of a pair of projections. Here we discuss a few illustrative cases for small $r$, $s$ and in particular we connect our theory of Clifford covariant Fredholm pairs to the classical theory of Fredholm pairs of orthogonal projections in a real or complex Hilbert space.

4.2.1. $r=0, s=0$. An element $J \in J^{0,1}(H)$ is just a complex structure without any additional symmetries. Thus, if $\left(\mathrm{J}_{0}, \mathrm{~J}_{1}\right)$ is a Fredholm pair in $\mathrm{g}^{0,1}(\mathrm{H})$ then by the discussion in Sec. 2.4.4 we infer that $\operatorname{Ind}_{0,2}\left(\mathrm{~J}_{0}, \mathrm{~J}_{1}\right)=\frac{1}{2} \operatorname{dim} \operatorname{ker}\left(\mathrm{J}_{0}+\mathrm{J}_{1}\right)(\bmod 2)$. Note that $\operatorname{ker}\left(\mathrm{J}_{0}+\mathrm{J}_{1}\right)$ is invariant under $\mathrm{J}_{0}$ and is therefore automatically even, cf. [CPSB19, Prop. 6.2].

We compare $\operatorname{Ind}_{0,2}$ to $\mathrm{Sf}_{2}$ of [CPSB19, Def. 2.1]:

Proposition 4.8. Let $\mathrm{H}$ be a finite dimensional Hilbert space, $\mathrm{J} \in \mathcal{J}^{0,1}(\mathrm{H})$, and $\mathrm{O} \in \mathrm{O}(\mathrm{H})$ orthogonal. Then

$$
(-1)^{\operatorname{Ind}_{0,2}\left(\mathrm{~J}, \mathrm{O}^{*} \mathrm{JO}\right)}=\operatorname{det}(\mathrm{O}),
$$

thus $\mathrm{Ind}_{0,2}$ coincides with $\mathrm{Sf}_{2}$ of [CPSB19, Def. 2.1].

Proof. Both sides are locally constant in O. Furthermore, all $J \in J^{0,1}(H)$ are unitarily equivalent. Therefore, it suffices to check this for $\mathrm{O}=\mathrm{I}$ in which case both sides are 1 and for

$$
\mathrm{O}=\left(\begin{array}{ll}
0 & 1 \\
1 & 0
\end{array}\right), \quad J=\left(\begin{array}{cc}
0 & -1 \\
1 & 0
\end{array}\right)
$$

in which case both sides equal -1 . 
4.2.2. $r=1, s=0$. Let $\mathrm{H}$ be a $\mathrm{C}_{r, s}$-Hilbert space. With regard to Prop. 2.3 and the second part of Remark 2.4, we may write $H=H^{\prime} \oplus H^{\prime}$ such that $E_{1}=K_{1}$. If $J \in J^{1,1}(H)$ then $J$ takes the form

$$
\mathrm{J}=\left(\begin{array}{cc}
0 & -\mathrm{U}^{*} \\
\mathrm{U} & 0
\end{array}\right)=\mathfrak{R}(\mathrm{U}) \otimes \mathrm{L}_{1}+\mathfrak{I}(\mathrm{U}) \otimes \mathrm{K}_{2} \text {. }
$$

Thus $\mathrm{U}=\mathrm{U}(\mathrm{J})$ is a real unitary (aka orthogonal) operator. If $\left(\mathrm{J}_{0}, \mathrm{~J}_{1}\right)$ is a Fredholm pair in $g^{1,1}(\mathrm{H})$ and if $\mathrm{U}_{\mathrm{k}}:=\mathrm{U}\left(\mathrm{J}_{\mathrm{k}}\right), \mathrm{k}=0,1$ denotes the associated unitary operators, then comparison with Sec. 2.4.3 shows that

$$
\begin{aligned}
\operatorname{Ind}_{1,2}\left(J_{0}, J_{1}\right)=\frac{1}{2} & \operatorname{dim} \operatorname{ker}\left(J_{0}+J_{1}\right)(\bmod 2) \\
& =\operatorname{dim} \operatorname{ker}\left(\mathrm{U}_{0}+\mathrm{U}_{1}\right)(\bmod 2)=\operatorname{dim} \operatorname{ker}\left(\mathrm{I}+\mathrm{U}_{0}^{*} \mathrm{U}_{1}\right)(\bmod 2) .
\end{aligned}
$$

Note that $\left(\mathrm{J}_{0}, \mathrm{~J}_{1}\right)$ being a Fredholm pair is equivalent to $\left\|\mathrm{U}_{0}-\mathrm{U}_{1}\right\|_{Q}<2$ which in turn, cf. Eq. (3.3), is equivalent to $\mathrm{I}+\mathrm{U}_{0}^{*} \mathrm{U}_{1}$ being Fredholm. Furthermore, since $\mathrm{U}_{0}, \mathrm{U}_{1}$ are real, eigenvalues of $\mathrm{U}_{0}^{*} \mathrm{U}_{1}$ moving through -1 come in pairs, hence dim $\operatorname{ker}\left(I+U_{0}^{*} U_{1}\right)(\bmod 2)$ has the stability properties of a Fredholm index.

We note that $\operatorname{Ind}_{1,2}\left(\mathrm{~J}_{0}, \mathrm{~J}_{1}\right)$ equals the parity index defined in [DSBW19, Def. 3]. By Theorem 2.6 and Prop. 2.9 (or just directly) and suppressing forgetful maps from the notation, we have

$$
\operatorname{Ind}_{1,2}\left(J_{0}, J_{1}\right)=\operatorname{Ind}_{0,2}\left(J_{0}, J_{1}\right) \text {. }
$$

Proposition 4.9. Let $\mathrm{H}$ be a finite dimensional Hilbert space, $\mathrm{J}_{\mathrm{k}}=\left(\begin{array}{cc}0 & -\mathrm{U}_{k}^{*} \\ \mathrm{U}_{\mathrm{k}} & 0\end{array}\right) \in$ $\mathrm{g}^{1,1}(\mathrm{H}), \mathrm{k}=0,1$ with associated orthogonal matrices $\mathrm{U}_{0}, \mathrm{U}_{1} \in \mathrm{O}(\mathrm{H})$. Then

$$
(-1)^{\operatorname{dim} \operatorname{ker}\left(\mathrm{I}+\mathrm{U}_{0}^{*} \mathrm{U}_{1}\right)}=\operatorname{det}\left(\mathrm{U}_{0}^{*} \mathrm{U}_{1}\right)=\left(\operatorname{det} \mathrm{U}_{0}\right) \cdot\left(\operatorname{det} \mathrm{U}_{1}\right) .
$$

This should be compared to [DSBW19, Def. 1].

Proof. The proof is similar to the proof of Prop. 4.8.

4.2.3. $\mathrm{r}=2, \mathrm{~s}=0$ : the index of a pair of projections. Let $\mathrm{H}$ be a $\mathrm{Cl}_{r, s}-$ Hilbert space. Apply Prop. 2.3 and write $\mathrm{H}=\mathrm{H}^{\prime} \oplus \mathrm{H}^{\prime}$ such that

$$
\mathrm{E}_{1}=\mathrm{K}_{1}=\left(\begin{array}{cc}
1 & 0 \\
0 & -1
\end{array}\right), \quad \mathrm{E}_{2}=\mathrm{K}_{2}=\left(\begin{array}{ll}
0 & 1 \\
1 & 0
\end{array}\right) .
$$

If $J \in g^{2,1}(H)$ then $J$ takes the form

$$
\left(\begin{array}{cc}
0 & -(2 \mathrm{P}-\mathrm{I}) \\
2 \mathrm{P}-\mathrm{I} & 0
\end{array}\right)
$$

with an orthogonal projection ${ }^{8} \mathrm{P} \in \mathcal{B}\left(\mathrm{H}^{\prime}\right)$. Thus there is a one to one correspondence between Fredholm pairs $\left(\mathrm{J}_{0}, \mathrm{~J}_{1}\right)$ in $\mathcal{f}^{2,1}(\mathrm{H})$ and pairs of orthogonal projections (without further symmetries) $\mathrm{P}, \mathrm{Q} \in \mathcal{B}\left(\mathrm{H}^{\prime}\right)$ with $\|\mathrm{P}-\mathrm{Q}\|_{\mathcal{Q}\left(\mathrm{H}^{\prime}\right)}<1$.

Let us therefore recall that a pair of orthogonal projections $\mathrm{P}, \mathrm{Q}$ acting on a separable real or complex Hilbert space $\mathrm{H}$ is called a Fredholm pair if $\|\mathrm{P}-\mathrm{Q}\|_{\mathcal{Q}}<$

\footnotetext{
${ }^{8} \mathrm{~A}$ linear operator $\mathrm{P} \in \mathcal{B}(\mathrm{H})$ is an orthogonal projection if $\mathrm{P}^{*}=\mathrm{P}=\mathrm{P}^{2}$.
} 
1. This is equivalent to the fact that $\left.Q\right|_{\operatorname{ran} P}: \operatorname{ran} P \rightarrow \operatorname{ran} Q$ is a Fredholm operator. The index of this Fredholm operator is called the index ind $(P, Q)$ of the pair.

Lemma 4.10 (cf. [ASS94A, Prop. 3.1] ). Let P, Q be a Fredholm pair of orthogonal projections. Then $\operatorname{ker}(\mathrm{P}+\mathrm{Q}-\mathrm{I})=\operatorname{ran} \mathrm{P} \cap \operatorname{ker} \mathrm{Q} \oplus \operatorname{ker} \mathrm{P} \cap \operatorname{ran} \mathrm{Q}$ and $\operatorname{ind}(\mathrm{P}, \mathrm{Q})=$ $\operatorname{dim}(\operatorname{ran} \mathrm{P} \cap \operatorname{ker} \mathrm{Q})-\operatorname{dim}(\operatorname{ker} \mathrm{P} \cap \operatorname{ran} \mathrm{Q})$.

Proof. If $x \in \operatorname{ker}(\mathrm{P}+\mathrm{Q}-\mathrm{I})$ then $\mathrm{P} x=(\mathrm{I}-\mathrm{Q}) x \in \operatorname{ran} \mathrm{P} \cap \operatorname{ker} \mathrm{Q} \operatorname{resp} . \mathrm{Q} x=(\mathrm{I}-\mathrm{P}) x \in$ ker $P \cap \operatorname{ran} Q$, proving the inclusion $\subset$. The inclusion $\supset$ is similarly easy. To see the index formula, one notes that the adjoint of the operator $\left.\mathrm{Q}\right|_{\operatorname{ran} P}: \operatorname{ran} \mathrm{P} \rightarrow \operatorname{ran} \mathrm{Q}$ is given by $\left.\mathrm{P}\right|_{\text {ran } \mathrm{Q}}: \operatorname{ran} \mathrm{Q} \rightarrow \operatorname{ran} \mathrm{P}$.

Let us return to the $\mathrm{Cl}_{2,0}$-Hilbert space $\mathrm{H}=\mathrm{H}^{\prime} \otimes \mathbb{R}^{2}$ as before and consider a Fredholm pair $\left(\mathrm{J}_{0}, \mathrm{~J}_{1}\right)$ in $\mathcal{J}^{2,1}(\mathrm{H})$ with associated orthogonal projections $\mathrm{P}_{0}, \mathrm{P}_{1} \in$ $\mathcal{B}(\mathrm{H})$ such that

$$
\mathrm{J}_{l}=\left(\begin{array}{cc}
0 & -\left(2 \mathrm{P}_{\mathrm{l}}-\mathrm{I}\right) \\
2 \mathrm{P}_{\mathrm{l}}-\mathrm{I} & 0
\end{array}\right), \mathrm{l}=0,1 .
$$

We have seen that $\left(J_{0}, J_{1}\right)$ being a Fredholm pair is equivalent to $\left(P_{0}, P_{1}\right)$ being a Fredholm pair of orthogonal projections. To compute the index $\operatorname{ind}_{2,2}\left(\mathrm{~J}_{0}, \mathrm{~J}_{1}\right)$ we apply Sec. 2.4.2, by first computing the volume element of the $\mathrm{Cl}_{2,1}$ representation given by $E_{1}, E_{2}, J_{0}$,

$$
\omega=E_{1} \cdot E_{2} \cdot J_{0}=\left(\begin{array}{cc}
1 & 0 \\
0 & -1
\end{array}\right) \cdot\left(\begin{array}{ll}
0 & 1 \\
1 & 0
\end{array}\right) \cdot\left(\begin{array}{cc}
0 & -(2 P-I) \\
2 P-I & 0
\end{array}\right)=\left(\begin{array}{cc}
2 P-I & 0 \\
0 & 2 P-I
\end{array}\right) \cdot
$$

Furthermore in view of Lemma 4.10 we have

$$
\begin{aligned}
\operatorname{ker}\left(\mathrm{J}_{0}+\mathrm{J}_{1}\right)= & \operatorname{ran} \mathrm{P} \cap \operatorname{ker} \mathrm{Q} \oplus \operatorname{ker} \mathrm{P} \cap \operatorname{ran} \mathrm{Q} \\
& \operatorname{ran} \mathrm{P} \cap \operatorname{ker} \mathrm{Q} \oplus \operatorname{ker} \mathrm{P} \cap \operatorname{ran} \mathrm{Q} \\
= & (\operatorname{ran} \mathrm{P} \cap \operatorname{ker} \mathrm{Q}) \otimes \mathbb{R}^{2} \oplus(\operatorname{ker} \mathrm{P} \cap \operatorname{ran} \mathrm{Q}) \otimes \mathbb{R}^{2} .
\end{aligned}
$$

With respect to this decomposition, $\omega$ acts on $\operatorname{ker}\left(J_{0}+J_{1}\right)$ as

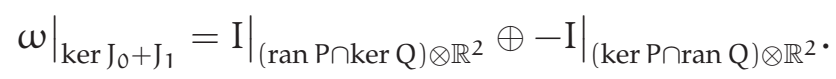

Now $\operatorname{ind}_{2,2}\left(\mathrm{~J}_{0}, \mathrm{~J}_{1}\right)$ is the class of the $\mathrm{Cl}_{2,1}$ module $\operatorname{ker}\left(\mathrm{J}_{0}, \mathrm{~J}_{1}\right)$ with Clifford generators $E_{1}, E_{2}, F_{1}=J_{0}$. By Sec. 2.4.2 its class in $A_{2,2}$ is naturally identified with the number

$$
\frac{1}{2}\left(\operatorname{dim} \operatorname{ker}\left(\left.\omega\right|_{\operatorname{ker}\left(J_{0}+J_{1}\right)}-I\right)-\operatorname{dim} \operatorname{ker}\left(\left.\omega\right|_{\operatorname{ker}\left(J_{0}+J_{1}\right)}+I\right)\right) \in \mathbb{Z}
$$

Thus

$$
\begin{aligned}
\operatorname{Ind}_{2,2}\left(J_{0}, J_{1}\right)= & \frac{1}{2} \operatorname{dim}\left(\operatorname{ker}\left(J_{0}+J_{1}\right) \cap \operatorname{ker}(\omega-I)\right) \\
& \quad-\frac{1}{2} \operatorname{dim}\left(\operatorname{ker}\left(J_{0}+J_{1}\right) \cap \operatorname{ker}(\omega+I)\right) \\
= & \operatorname{dim}(\operatorname{ran} P \cap \operatorname{ker} Q)-\operatorname{dim}(\operatorname{ker} P \cap \operatorname{ran} Q) \\
= & \operatorname{ind}(Q: \operatorname{ran} P \rightarrow \operatorname{ran} Q)=\operatorname{ind}(P, Q) .
\end{aligned}
$$


The previous discussion also can be applied to the forgetful maps considered in Theorem 2.6 and Prop. 2.9. Namely, suppressing the forgetful maps from the notation, we have

$$
\operatorname{Ind}_{0,2}\left(J_{0}, J_{1}\right)=\operatorname{Ind}_{1,2}\left(J_{0}, J_{1}\right)=\operatorname{Ind}_{2,2}\left(J_{0}, J_{1}\right)(\bmod 2) .
$$

4.2.4. $r=0, s=0$ in the complex case. We briefly mention the case of a complex Hilbert space $\mathrm{H}$ where things are easier as there is a direct correspondence between "complex structures" and orthogonal projections since we may write $J=i(2 P-I)$. Therefore, there is no need to introduce additional symmetries.

Given orthogonal projections $\mathrm{P}, \mathrm{Q}$ put $\mathrm{J}_{0}:=i(2 \mathrm{P}-\mathrm{I}), \mathrm{J}_{1}=i(2 \mathrm{Q}-\mathrm{I})$. Then $\mathrm{J}_{0}, \mathrm{~J}_{1} \in \mathrm{J}^{0,1}(\mathrm{H})$ and $\left\|\mathrm{J}_{0}-\mathrm{J}_{1}\right\|_{Q}=2\|\mathrm{P}-\mathrm{Q}\|_{Q}$. Hence $\left(\mathrm{J}_{0}, \mathrm{~J}_{1}\right)$ is a Fredholm pair in the sense of Def. 4.1 if and only if $(P, Q)$ is a Fredholm pair of projections. Furthermore,

$$
\frac{1}{2}\left(J_{0}+J_{1}\right)=i(P+Q-I)
$$

thus by the Lemma 4.10 we have

$$
\operatorname{ker} \mathrm{J}_{0}+\mathrm{J}_{1}=\operatorname{ran} \mathrm{P} \cap \operatorname{ker} \mathrm{Q} \oplus \operatorname{ker} \mathrm{P} \cap \operatorname{ran} \mathrm{Q} .
$$

With respect to this decomposition, $\mathrm{J}_{0}$ acts as

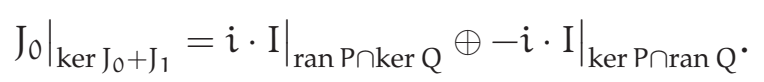

The class $\operatorname{ind}_{0,2}\left(J_{0}, J_{1}\right)$ is the class of the $\mathbb{C} \ell_{1}$-module $\operatorname{ker}\left(J_{0}, J_{1}\right)$ with Clifford generator $E_{1}=J_{0}$. The class of an ungraded $\mathbb{C} \ell_{1}$-module $\left(V, E_{1}\right)$ in $\mathcal{M}_{0,1} / \mathcal{M}_{0,2} \simeq \mathbb{Z}$ is naturally identified (cf. Sec. 2) with the number

$$
\operatorname{dim} \operatorname{ker}\left(E_{1}-\mathfrak{i}\right)-\operatorname{dim} \operatorname{ker}\left(E_{1}+\mathfrak{i}\right) \in \mathbb{Z} \text {. }
$$

Thus

$$
\begin{aligned}
\operatorname{Ind}_{2}\left(J_{0}, J_{1}\right) & =\operatorname{dim}\left(\operatorname{ker}\left(J_{0}+J_{1}\right) \cap \operatorname{ker}\left(J_{0}-i\right)\right)-\operatorname{dim}\left(\operatorname{ker}\left(J_{0}+J_{1}\right) \cap \operatorname{ker}\left(J_{0}+i\right)\right) \\
& =\operatorname{dim}(\operatorname{ran} P \cap \operatorname{ker} Q)-\operatorname{dim}(\operatorname{ker} P \cap \operatorname{ran} Q) \\
& =\operatorname{ind}(Q: \operatorname{ran} P \rightarrow \operatorname{ran} Q)=\operatorname{ind}(P, Q) .
\end{aligned}
$$

4.3. Standard forms of complex structures. Using the ABS construction of Sec. 2, we now give standard generators of complex structures with a non-trivial index. These pairs will be constructed to represent an arbitrary class in $\mathrm{KO}_{s+2-r}(\mathbb{R})$ (resp. an arbitrary ind $_{r, s+2}$ ), cf. Cor. 4.6 and the proof of Theorem 4.5 . This will be needed in particular for the formulation of the axioms which ensure the uniqueness of the KO-valued spectral flow, see Remark 5.2 5. and Sec. 8.3 below.

Proposition 4.11. Let $\mathrm{V}$ be a finite dimensional $\mathrm{C}_{r, s+1}$-module with Clifford generators $\mathrm{E}_{1}, \ldots, \mathrm{E}_{\mathrm{r}}, \mathrm{F}_{1}, \ldots, \mathrm{F}_{\mathrm{s}+1}$ representing a class in $\mathrm{A}_{\mathrm{r}, \mathrm{s}+2}$. Let $\mathrm{H}_{0}$ be a standard $\mathrm{Cl}_{\mathrm{r}, \mathrm{s}+1}$ Hilbert space and $\mathrm{H}:=\mathrm{H}_{0} \oplus \mathrm{V}$. Now put

$$
\mathrm{J}_{0}:=\mathrm{F}_{\mathrm{s}+1}=\left.\left.\mathrm{F}_{\mathrm{s}+1}\right|_{\mathrm{H}_{0}} \oplus \mathrm{F}_{\mathrm{s}+1}\right|_{\mathrm{V}}, \quad \mathrm{J}_{1}:=\left.\mathrm{F}_{\mathrm{s}+1}\right|_{\mathrm{H}_{0}} \oplus-\left.\mathrm{F}_{\mathrm{s}+1}\right|_{\mathrm{V}} .
$$

Then $\left(\mathrm{J}_{0}, \mathrm{~J}_{1}\right)$ is a Fredholm pair of elements of $\mathrm{g}^{\mathrm{r}, \mathrm{s}+1}(\mathrm{H})$ with $\operatorname{ind}_{\mathrm{r}, \mathrm{s}+2}\left(\mathrm{~J}_{0}, \mathrm{~J}_{1}\right)=[\mathrm{V}]$. 
Proof. The difference $J_{0}-J_{1}$ is finite-rank, hence $\left\|J_{0}-J_{1}\right\|_{Q}=0$ and $\left(J_{0}, J_{1}\right)$ is a Fredholm pair. Furthermore, $\operatorname{ker}\left(\mathrm{J}_{0}+\mathrm{J}_{1}\right)=\mathrm{V}$ with $\mathrm{C}_{\mathrm{r}, \mathrm{s}+1}$ structure given by $\left.\mathrm{E}_{1}\right|_{V}, \ldots,\left.\mathrm{E}_{\mathrm{r}}\right|_{V},\left.\mathrm{~F}_{1}\right|_{V}, \ldots,\left.\mathrm{F}_{\mathrm{s}+1}\right|_{V}$, hence $\operatorname{ind}_{\mathrm{r}, \mathrm{s}+2}\left(\mathrm{~J}_{0}, \mathrm{~J}_{1}\right)=[\mathrm{V}]$.

\section{The KO-Valued SPectral Flow}

This section is the core of the paper. We give the definition of $\mathrm{KO}-$ valued spectral flow along with its basic properties. We subsequently relate our definition to the approach of J. Phillips [PHI96] and more generally show how our construction encompasses previous instances of analytic spectral flow that have appeared in the literature. Unless otherwise said, $\mathrm{H}$ denotes a fixed $\mathrm{C}_{r, s}-$ Hilbert space.

5.1. Definition and properties. We can now define the $A_{r, s+2} \simeq \mathcal{M}_{r, s+1} / \mathcal{M}_{r, s+2} \simeq$ $\mathrm{KO}_{\mathrm{r}, \mathrm{s}+2}(\mathbb{R})$-valued spectral flow of a path in $\mathcal{F}^{\mathrm{r}, \mathrm{s}+1}$ with invertible endpoints exactly as it was done in [BBLPo5, Leso5]:

Definition 5.1. Let $[0,1] \ni t \mapsto \mathrm{T}_{\mathrm{t}} \in \mathcal{F}^{\mathrm{r}, \mathrm{s}+1}(\mathrm{H})$ be a continuous path of skew-adjoint Fredholm operators with invertible end points $\mathrm{T}_{0}, \mathrm{~T}_{1}$. Let $\Phi$ be the map defined in Eq. (3.7). Then

$$
\mathrm{t} \mapsto \Phi\left(\mathrm{T}_{\mathrm{t}}\right)=-\mathrm{F}_{\mathrm{s}}\left(\mathrm{I}+\mathrm{T}_{\mathrm{t}} \mathrm{F}_{\mathrm{s}}\right)\left(\mathrm{I}-\mathrm{T}_{\mathrm{t}} \mathrm{F}_{\mathrm{s}}\right)^{-1}
$$

is a path $([0,1],\{0,1\}) \rightarrow\left(\widetilde{\Omega}_{r, s}, \widetilde{\Omega}_{r, s, *}\right)$ in $\widetilde{\Omega}_{r, s}$ with endpoints in the contractible (see Lemma 3.3) neighbourhood $\widetilde{\Omega}_{r, s, *}$ of $F_{s}$. Connect the endpoints of $\Phi\left(T_{\bullet}\right)$ arbitrarily, within $\widetilde{\Omega}_{r, s, *}$, to $F_{s}$. Then let

$$
\operatorname{sf}_{r, s+2}\left(T_{\bullet}\right) \in A_{r, s+2} \simeq K_{s+2-r}(\mathbb{R})
$$

be the class of the resulting loop in the stable fundamental group $\pi_{1}^{\mathrm{S}}\left(\widetilde{\Omega}_{\mathrm{r}, \mathrm{s}}, \mathrm{F}_{\mathrm{s}}\right)$. Since $\widetilde{\Omega}_{\mathrm{r}, \mathrm{s}, *}$ is contractible, it is irrelevant how the path was closed up. Alternatively, embed the Hilbert space $\mathrm{H}$, equipped with the $\mathrm{C}_{r, s+1}$ structure with generators $\mathrm{E}_{1}, \ldots, \mathrm{E}_{\mathrm{r}+\mathrm{s}}, \mathrm{T}_{0}\left|\mathrm{~T}_{0}\right|^{-1}$, into a standard $\mathrm{Cl}_{r, s+1}$-Hilbert space $\mathrm{H}^{\prime}$ and take the class of the loop obtained from $\Phi\left(\mathrm{T}_{\mathrm{t}} \oplus\right.$ $\left.\left.\mathrm{F}_{\mathrm{s}+1}\right|_{\mathrm{H}^{\perp}}\right)$ in the fundamental group $\pi_{1}\left(\widetilde{\Omega}_{\mathrm{r}, \mathrm{s}}\left(\mathrm{H}^{\prime}\right), \mathrm{F}_{\mathrm{s}}\right)$.

Finally, we put $\mathrm{SF}_{\mathrm{r}, \mathrm{s}+2}\left(\mathrm{~T}_{\bullet}\right):=\tau_{\mathrm{r}, \mathrm{s}+2}\left(\mathrm{sf}_{\mathrm{r}, \mathrm{s}+2}\left(\mathrm{~T}_{\bullet}\right)\right)$ with $\tau_{\mathrm{r}, \mathrm{s}+2}$ as in $\mathrm{Eq}$. (2.17).

Remark 5.2. It follows from the Absorption Theorem mentioned in Sec. 2.1 that the $\mathrm{sf}_{\mathrm{r}, \mathrm{s}+2}$ is well-defined. We collect some properties of the spectral flow. These properties will be of relevance for the discussion of the uniqueness in Sec. 7 below.

1. Relation to the index of Fredholm pairs of complex structures. Let $\mathrm{J}_{0}, \mathrm{~J}_{1} \in \mathrm{g}^{\mathrm{r}, \mathrm{s}+1}(\mathrm{H})$ be a Fredholm pair of complex structures. It then follows from Definition and Proposition 4.3 and Theorem 3.4 that ind $\mathrm{r}_{\mathrm{r}, \mathrm{s}+2}\left(\mathrm{~J}_{0}, \mathrm{~J}_{1}\right)$ equals the spectral flow $\mathrm{sf}_{\mathrm{r}, \mathrm{s}+2}$ of the straight line path $t \mapsto(1-t) J_{0}+t J_{1}$.

2. Homotopy invariance. If $\mathrm{T}:[0,1] \times[0,1] \rightarrow \mathcal{F}^{\mathrm{r}, s+1}(\mathrm{H})$ is a continuous map such that for all $u \in[0,1]$ the endpoints $T(0, u), T(1, u)$ are invertible operators, then $\mathrm{sf}_{\mathrm{r}, \mathrm{s}+2}(\mathrm{~T}(\cdot, 0))=\mathrm{sf}_{\mathrm{r}, \mathrm{s}+2}(\mathrm{~T}(\cdot, 1))$.

To see this, consider $\Phi(T(t, u))$. Now by Lemma 3.3 and the proof of Prop. 3.2 it follows that the endpoints $\Phi(T(0, u)), \Phi(T(1, u))$ can be connected to $F_{s}$ continuously in the parameter $u$ to obtain a homotopy of loops. Hence by definition $\operatorname{sf}_{\mathrm{r}, \mathrm{s}+2}(\mathrm{~T}(\cdot, 0))=\mathrm{sf}_{\mathrm{r}, \mathrm{s}+2}(\mathrm{~T}(\cdot, 1))$. 
3. Path additivity. If $\mathrm{T}_{\bullet}^{1}, \mathrm{~T}_{\bullet}^{2}:[0,1] \rightarrow \mathcal{F}^{\mathrm{r}, \mathrm{s}+1}(\mathrm{H})$ are paths of Fredholm operators with invertible endpoints and $T_{1}^{1}=T_{0}^{2}$ then the $\mathrm{sf}_{\mathrm{r}, \mathrm{s}+2}$ of the concatenated path $\mathrm{T}^{1} * \mathrm{~T}^{2}$ equals the $\mathrm{sf}_{\mathrm{r}, \mathrm{s}+2}\left(\mathrm{~T}_{\bullet}^{1}\right)+\mathrm{sf}_{\mathrm{r}, \mathrm{s}+2}\left(\mathrm{~T}_{\bullet}^{2}\right)$.

4. Stability. Let $\mathrm{T}_{\bullet}:[0,1] \rightarrow \mathcal{F}^{\mathrm{r}, \mathrm{s}+1}(\mathrm{H})$ be a path of Fredholm operators with invertible endpoints. Let $\mathrm{H}^{\prime}$ be another $C \ell_{r, s}-$ Hilbert space and $S \in \mathcal{F}^{r, s+1}\left(\mathrm{H}^{\prime}\right)$ a fixed invertible operator. Then $\mathrm{sf}_{\mathrm{r}, s+2}\left(\mathrm{~T}_{\bullet}\right)=\mathrm{sf}_{\mathrm{r}, s+2}\left(\mathrm{~T}_{\bullet} \oplus S\right)$.

5. Normalization. Let $\mathrm{V}$ be a finite dimensional $\mathrm{C}_{\mathrm{r}, s+1}-$ module (cf. Sec. 4.3) and consider the straight line path $\gamma(t)=(1-2 t) F_{s+1}, 0 \leq t \leq 1$ from $F_{s+1}$ to $-F_{s+1}$. Then

$$
\operatorname{sf}_{r, s+2}(\gamma)=[\mathrm{V}] \in A_{r, s+2} \simeq \mathcal{M}_{r, s+1} / \mathcal{M}_{r, s+2} .
$$

This follows immediately from the relation to the index of a pair since here $\operatorname{sf}_{r, s+2}(\gamma)=\operatorname{ind}_{r, s+2}\left(F_{s+1},-F_{r, s+1}\right)=\left[\operatorname{ker}\left(F_{r, s+1}+\left(-F_{r, s+1}\right)\right)\right]=[V]$.

6. Constancy. The $\mathrm{sf}_{\mathrm{r}, \mathrm{s}+2}(\mathrm{~T})$ of a constant path (in which $\mathrm{T}$ must be invertible) is 0 .

7. Direct sum. If $\mathrm{T}_{0}^{1}:[0,1] \rightarrow \mathcal{F}^{\mathrm{r}, \mathrm{s}+1}\left(\mathrm{H}_{1}\right), \mathrm{T}_{\bullet}^{2}:[0,1] \rightarrow \mathcal{F}^{\mathrm{r}, \mathrm{s}+1}\left(\mathrm{H}_{2}\right)$ are paths of skew-adjoint Fredholm operators with invertible endpoints, then $\mathrm{sf}_{\mathrm{r}, \mathrm{s}+2}\left(\mathrm{~T}_{\bullet}^{1}\right)+$ $\mathrm{sf}_{\mathrm{r}, \mathrm{s}+2}\left(\mathrm{~T}_{\bullet}^{2}\right)=\mathrm{sf}_{\mathrm{r}, \mathrm{s}+2}\left(\mathrm{~T}_{\bullet}^{1} \oplus \mathrm{T}_{\bullet}^{2}\right)$.

5.2. The 'local formula' a la J. Phillips. Next we will show that the spectral flow of a path $T_{0}$ can be computed 'locally' along the path by measuring the relative indices of the phases of $T_{0}$. This is the analogue of J. Phillips [PHIg6] approach in the complex case.

Let $[0,1] \ni t \mapsto T_{t} \in \mathcal{F}^{r, s+1}(H)$ be a continuous path with $T_{0}, T_{1}$ being invertible. The first difficulty is that the path of phases $T_{t}\left|T_{t}\right|^{-1}$ is ill-defined as $T_{t}$ might not be invertible. However, the invertibility of the endpoints and the local constancy of the Fredholm index imply ind $\mathrm{r}_{\mathrm{r}, \mathrm{s}+1} \mathrm{~T}_{\mathrm{t}}=0$ for all $0 \leq \mathrm{t} \leq 1$. Hence there exists a complex structure $\mathrm{F}_{s+1} \in \mathcal{J}^{r, s+1}\left(\operatorname{ker} \mathrm{T}_{\mathrm{t}}\right)$ turning $\operatorname{ker} \mathrm{T}_{\mathrm{t}}$ into a $\mathrm{C} \ell_{\mathrm{r}, s+1}-$ module. We denote by $J\left(T_{t}\right) \in \mathcal{J}^{r, s+1}(H)$ a complex structure with

$$
\begin{aligned}
\left.J\left(T_{t}\right)\right|_{\text {ker } T_{t}^{+}} & =T_{t}\left|T_{t}\right|^{-1}, \\
\left.J\left(T_{t}\right)\right|_{\text {ker } T_{t}} & \in \mathcal{J}^{r, s+1}\left(\operatorname{ker} T_{t}\right) \text { arbitrary . }
\end{aligned}
$$

The complex structure $J\left(T_{t}\right)$ is unique only up to a finite rank perturbation, but certainly $t \mapsto \pi\left(J\left(T_{t}\right)\right) \in \mathcal{Q}(H)$ is continuous in the Calkin algebra.

Alternatively, one might require, analogously to [CPSB19, Sec. 4], that $J\left(T_{t}\right)=$ $T_{t}\left|T_{t}\right|^{-1}$ only on the complement of a spectral subspace ran $\chi_{(0, a)}\left(\left|T_{t}\right|\right)$, where $0<$ $a<\min$ specess $_{\text {es }}\left|T_{t}\right|$. This would allow for example that $t \mapsto J\left(T_{t}\right)$ has only finitely many discontinuities. The next Lemma says that given a subdivision of the interval $[0,1]$, there is a finite-rank perturbation $\widetilde{T}_{\bullet}$ of $T_{0}$ with the same spectral flow and such that $T_{t}$ is invertible at the subdivision points, moreover the phases may be prescribed.

Lemma 5.3. Let $T_{t}$ and $J_{t}:=J\left(T_{t}\right)$ be as before and let $0=t_{0}<t_{1} \cdots<t_{n}=1$ be a subdivision of the interval $[0,1]$. Then there exists a continuous path $[0,1] \ni t \mapsto \widetilde{T}_{t} \in$ $\mathcal{F}^{\mathrm{r}, \mathrm{s}+1}(\mathrm{H})$ with the following properties: 
(1) $\widetilde{T}_{t}-T_{t}$ is finite rank for all $t$.

(2) $\widetilde{T}_{t_{j}}$ is invertible and $\widetilde{T}_{t_{j}}\left|\widetilde{T}_{t_{j}}\right|^{-1}=J_{t_{j}}$ for $j=0,1, \ldots, n$.

(3) $\mathrm{sf}_{\mathrm{r}, \mathrm{s}+1}\left(\widetilde{\mathrm{T}}_{\bullet}\right)=\mathrm{sf}_{\mathrm{r}, \mathrm{s}+1}\left(\mathrm{~T}_{\bullet}\right)$.

Proof. For each $j=1, \ldots, n-1$ let, e.g.,

$$
\begin{cases}\left.\mathrm{R}_{\mathrm{j}}\right|_{\operatorname{ker}_{\mathrm{t}_{j}}}:=\left.\mathrm{J}_{\mathrm{t}_{\mathrm{j}}}\right|_{\operatorname{ker}_{\mathrm{t}_{\mathrm{j}}}}, \\ \left.\mathrm{R}_{\mathrm{j}}\right|_{\text {ker } \mathrm{T}_{\mathrm{t}_{j}}^{\perp}}:=0 .\end{cases}
$$

Furthermore let $\varphi_{j} \in \mathrm{C}^{\infty}[0,1]$ be smooth bump functions supported in a neighbourhood of $t_{j}$ resp. such that their supports are disjoint and such that $\varphi_{j}\left(t_{j}\right)=1$. Then $\widetilde{T}_{t}:=T_{t}+\sum_{j=1}^{n-1} \varphi_{j}(t) R_{j}$ does the job.

Property (3) follows since $\widetilde{T}_{\bullet}$ and $T_{0}$ have the same endpoints and hence the straight line $s \mapsto(1-s) \widetilde{T}_{\bullet}+s T_{\bullet}$ is a homotopy between $\widetilde{T}_{\bullet}$ and $T_{\bullet}$ keeping endpoints fixed.

Theorem 5.4 (cf. [CPSB19, Prop. 6.3]). Let $[0,1] \ni t \mapsto \mathrm{T}_{\mathrm{t}} \in \mathcal{F}^{\mathrm{r}, \mathrm{s}+1}(\mathrm{H})$ be a continuous path with $\mathrm{T}_{0}, \mathrm{~T}_{1}$ being invertible. Let $\mathrm{J}_{\mathrm{t}}$ be complex structures obtained by complementing the phase $\mathrm{T}_{\mathrm{t}}\left|\mathrm{T}_{\mathrm{t}}\right|^{-1}$ on ker $\mathrm{T}_{\mathrm{t}}^{+}$by an arbitrary complex structure in $\mathrm{g}^{\mathrm{r}, \mathrm{s}+1}\left(\operatorname{ker} \mathrm{T}_{\mathrm{t}}\right), c f$. Eq. (5.2).

Choose a partition $0=t_{0}<t_{1}<\cdots<t_{n}=1$ with the property that on each segment $\left\|\mathrm{J}_{\mathrm{t}_{j-1}}-\mathrm{J}_{\mathrm{t}}\right\|_{2}<1, \mathrm{t}_{\mathrm{j}-1} \leq \mathrm{t} \leq \mathrm{t}_{\mathrm{j}}$. Then

$$
\operatorname{sf}_{r, s+2}\left(T_{\bullet}\right)=\sum_{j=1}^{n} \operatorname{ind}_{r, s+2}\left(J_{t_{j-1}}, J_{t_{j}}\right) .
$$

Proof. We make repeated use of the homotopy invariance of $\mathrm{sf}_{\mathrm{r}, \mathrm{s}+2}$ and of ind $_{\mathrm{r}, \mathrm{s}+2}$.

In view of Lemma 5.3 we may w.l.o.g. assume that $T_{t_{j}}$ is invertible for $j=$ $0, \ldots, n$.

Since $t \mapsto T_{t}$ is a continuous path of Fredholm operators we have

$$
r:=\min \left(\min _{t} \operatorname{spec}_{\text {ess }}\left|T_{t}\right|, \text { min spec }\left|T_{t_{0}}\right|, \ldots, \min \operatorname{spec}\left|T_{t_{n}}\right|\right)>0 .
$$

We certainly have $J\left(T_{t} / r\right)=J\left(T_{t}\right)$ and by the homotopy invariance $\mathrm{sf}_{\mathrm{r}, \mathrm{s}+2}\left(\mathrm{~T}_{\bullet}\right)=$ $\mathrm{sf}_{\mathrm{r}, \mathrm{s}+2}\left(\mathrm{~T}_{\bullet} / \mathrm{r}\right)$.

Thus without loss of generality it suffices to prove the Theorem for paths with min specess $_{\text {en }}\left|T_{t}\right| \geq 1$ and min spec $\left|T_{t_{j}}\right| \geq 1, j=0, \ldots, n$.

With $\Psi$ of Eq. (3.10) consider the homotopy

$$
\begin{aligned}
H(u, t) & :=(1-u) T_{t}+u \Psi\left(T_{t}\right)=h_{u}\left(T_{t}\right), \\
h_{u}(x) & :=(1-u) x+u \Psi(x) .
\end{aligned}
$$

For all $u$ the function $h_{u}$ is increasing and strictly increasing in a neighbourhood of 0 . So for all $u$ we have $\operatorname{ker} H(u, t)=\operatorname{ker} T_{u}$ and $J(H(u, t))=J\left(T_{t}\right)$. Furthermore, by construction $h_{1}\left(T_{t_{j}}\right)=T_{t_{j}}\left|T_{t_{j}}\right|^{-1}=J_{t_{j}}$.

Hence both sides of the claimed equation remain constant under the deformation $\mathrm{H}$. We are finally left to prove the claim for the special case that $\operatorname{spec}_{\text {ess }} T_{t} \subset$ $\{ \pm i\}$ for all $t$ and $T_{t_{j}}=J_{t_{j}}$. 
But then $T_{t}-J\left(T_{t}\right)$ is of finite rank and hence $\left\|T_{t}-T_{t_{j-1}}\right\|_{Q}=\left\|J_{T_{t}}-J_{T_{j-1}}\right\|_{Q}<1$ for all $t_{j-1} \leq t \leq t_{j}$. Now $u \mapsto(1-u)\left((1-t) J_{t_{j-1}}+t J_{t_{j}}\right)+u T_{t}$ is a homotopy between $T_{t}, t_{j-1} \leq t \leq t_{j}$ and the straight line path $(1-t) J_{t_{j-1}}+t J_{t_{j}}$. The endpoints are kept fixed under the homotopy. Consequently

$$
\operatorname{sf}_{r, s+2}\left(\left(T_{t}\right)_{t_{j-1} \leq t \leq t_{j}}\right)=\operatorname{sf}_{r, s+2}\left((1-t) J_{t_{j-1}}+t J_{t_{j}}, 0 \leq t \leq 1\right)=\operatorname{ind}_{r, s+2}\left(J_{t_{j-1}}, J_{t_{j}}\right) .
$$

The path additivity of the spectral flow then implies the claim.

The previous result allows us to relate the $\mathrm{KO}-$ valued spectral flow to the various known versions of spectral flow.

Theorem 5.5. Let $\mathrm{T}_{\bullet} \in \mathcal{F}^{\mathrm{r}, 1}(\mathrm{H})$ be a family of Fredholm operators with invertible endpoints. Let $\mathrm{K}_{1}, \mathrm{~K}_{2}, \mathrm{~L}_{1} \in \mathrm{M}(2, \mathbb{R})$ be the Clifford matrices Eq. (2.4).

1. $\mathrm{r}=$ 2. Write $\mathrm{H}=\mathrm{H}^{\prime} \otimes \mathbb{R}^{2}$ with $\mathrm{E}_{\mathrm{j}}=\mathrm{I}_{\mathrm{H}} \otimes \mathrm{K}_{\mathrm{j}}, \mathrm{j}=1,2$. Then $\mathrm{T}_{\mathrm{t}}=\widetilde{\mathrm{T}}_{\mathrm{t}} \otimes \mathrm{K}_{1} \mathrm{~K}_{2}{ }^{9}$ with a family $\widetilde{\mathrm{T}}_{\bullet}$ of self-adjoint Fredholm operators and $\left.\mathrm{SF}\left(\widetilde{\mathrm{T}}_{\bullet}\right)=\mathrm{SF}_{2,2}\left(\mathrm{~T}_{\bullet}\right)\right)$.

2. $\mathrm{r}=0$. In this case $\mathrm{T}_{0}$ is a family of skew-adjoint Fredholm operators without any additional symmetries. Furthermore, $\mathrm{SF}_{0,2}\left(\mathrm{~T}_{\bullet}\right)$ equals the $\mathbb{Z} / 2 \mathbb{Z}$-valued Spectral Flow $\mathrm{Sf}_{2}$ of [CPSB19].

3. $\mathrm{r}=1$. Write again $\mathrm{H}=\mathrm{H}^{\prime} \otimes \mathbb{R}^{2}$ with $\mathrm{E}_{1}=\mathrm{K}_{1}$. Then $\mathrm{T}_{\mathrm{t}}=\mathfrak{R}\left(\widetilde{\mathrm{T}}_{\mathrm{t}}\right) \otimes \mathrm{K}_{2}+\mathfrak{I}\left(\widetilde{\mathrm{T}}_{\mathrm{t}}\right) \otimes \mathrm{L}_{1}$ with a family $\widetilde{T}_{0}$ of Fredholm operators on $\mathrm{H}^{\prime}$. We have a forgetful map $\mathcal{F}^{1,2} \subset \mathcal{F}^{0,2}$ and $\mathrm{SF}_{1,2}\left(\mathrm{~T}_{\bullet}\right)=\mathrm{SF}_{0,2}\left(\mathrm{~T}_{\mathbf{0}}\right)=\mathrm{Sf}_{2}\left(\mathrm{~T}_{\mathbf{0}}\right)$

Moreover, $\mathrm{SF}_{1,2}\left(\mathrm{~T}_{\mathbf{0}}\right)$ equals the parity $\sigma\left(\widetilde{\mathrm{T}}_{\mathbf{0}}\right)$ of [DSBW19, Def. 3]. More concretely, given a family $\widetilde{\mathrm{T}}_{\bullet}$ of Fredholm operators in a Hilbert space $\mathrm{H}^{\prime}$, put

$$
\mathrm{T}_{\mathrm{t}}:=\mathfrak{R}\left(\widetilde{\mathrm{T}}_{\mathrm{t}}\right) \otimes \mathrm{K}_{2}+\mathfrak{I}\left(\widetilde{\mathrm{T}}_{\mathrm{t}}\right) \otimes \mathrm{L}_{1}=\left(\begin{array}{cc}
0 & -\widetilde{\mathrm{T}}_{\mathrm{t}}^{*} \\
\widetilde{\mathrm{T}}_{\mathrm{t}} & 0
\end{array}\right)
$$

then

$$
\sigma\left(\widetilde{T}_{\bullet}\right)=\mathrm{SF}_{1,2}\left(\mathrm{~T}_{\bullet}\right)=\mathrm{SF}_{0,2}\left(\mathrm{~T}_{\bullet}\right)=\mathrm{Sf}_{2}\left(\mathrm{~T}_{\bullet}\right) .
$$

Proof. This follows immediately from the local formula Theorem 5.4 and the corresponding statements for the index of a pair in Sec. 4. The claimed tensor product decompositions follow from Prop. 2.3, cf. also Remark 2.42.

5.3. Examples from topological insulators. Here we briefly outline some applications of our $\mathrm{KO}$-valued spectral flow to models in condensed matter physics. We first review results in [CPSB19] and building from this idea provide a (somewhat ad hoc) example, which by the normalization and homotopy invariance of spectral flow covers all remaining cases. We also outline the relationship between paths of Hamiltonians in symmetry class AII with the quaternionic spectral flow $\mathrm{sf}_{\mathrm{r}, \mathrm{r}+4} \in \mathrm{KO}_{4}(\mathbb{R})$.

${ }^{9}$ Recall $\mathrm{K}_{1} \mathrm{~K}_{2}$ is the volume element of $\mathrm{C}_{2,0}$. 
Example 5.6 (Kitaev chain, cf. Sec. 1o of [CPSB19]). We consider a one dimensional Hamiltonian with particle-hole symmetry on $\ell^{2}(\mathbb{Z}) \otimes \mathbb{C}^{2}$, where

$$
\mathbf{H}(\mu, w)=\frac{1}{2}\left(\begin{array}{cc}
-w\left(S+S^{*}\right)-\mu & -i w\left(S^{*}-S\right) \\
-\mathfrak{i} w\left(S^{*}-S\right) & w\left(S+S^{*}\right)+\mu
\end{array}\right), \quad \mu, w \in \mathbb{R}
$$

and $S$ the unilateral shift operator on $\ell^{2}(\mathbb{Z})$. We see that $\mathbf{H}(\mu, w)$ anti-commutes with the particle-hole involution $C=\mathfrak{c}\left(\mathrm{I}_{\ell^{2}(\mathbb{Z})} \otimes \mathrm{K}_{2}\right)$ with $\mathfrak{c}$ complex conjugation and $K_{2}$ from Eq. (2.4). For simplicity we will consider the case of $w=-1, \mu=0$, where the Hamiltonian is invertible. We furthermore condense our notation and write

$$
\mathbf{H}=\mathbf{H}(0,-1)=\widehat{S}+\widehat{S}^{*}, \quad \widehat{S}=S \otimes \frac{1}{2}\left(\begin{array}{cc}
1 & i \\
i & -1
\end{array}\right) .
$$

We now perturb this Hamiltonian by inserting a local flux through the unit cell at site 0 . Namely, we consider the path for $\alpha \in[0,1]$

$$
\mathbf{H}_{\alpha}=\hat{S}_{\alpha}+\hat{S}_{\alpha}^{*}, \quad \hat{S}_{\alpha}=S \otimes \frac{1}{2}\left(\begin{array}{cc}
1 & i \\
i & -1
\end{array}\right)+v_{1} v_{0}^{*} \otimes \frac{1}{2}\left(\begin{array}{cc}
e^{-i \pi \alpha}-1 & i\left(e^{i \pi \alpha}-1\right) \\
i\left(e^{-i \pi \alpha}-1\right) & -\left(e^{i \pi \alpha}-1\right)
\end{array}\right),
$$

where $v_{n}$ is the partial isometry onto the site $n \in \mathbb{Z}$.

We see that $\mathbf{H}_{\alpha}-\mathbf{H}$ is finite-rank and therefore compact for any $\alpha$. We do not consider any additional symmetries or Clifford generators and so can compute $\mathrm{SF}_{0,2}\left(i \mathrm{H}_{\bullet}\right)=\mathrm{SF}_{2}\left(\mathrm{iH}_{\bullet}\right)$ using the $\mathrm{Cl}_{0,2}$ index of the (invertible) endpoints. Letting $\mathrm{J}_{\mathrm{k}}=i \mathbf{H}_{\mathrm{k}}\left|\mathbf{H}_{\mathrm{k}}\right|^{-1}$ for $\mathrm{k} \in 0,1$, it is shown in [CPSB19, Sec. 10] that

$$
\mathrm{SF}_{0,2}\left(i \mathrm{H}_{\bullet}\right)=\operatorname{ind}_{0,2}\left(\mathrm{~J}_{0}, \mathrm{~J}_{1}\right)=1 \text {. }
$$

Example 5.7 (KO-valued spectral flow in the Kitaev chain). Let us follow the same basic idea of Example 5.6 and consider more general $\mathrm{KO}$-valued spectral flow via a flux insertion of a one dimensional Hamiltonian. Let $\mathrm{V}$ be a finite dimensional $\mathrm{C}_{r, s+1}$-module with Clifford generators $\mathrm{E}_{1}, \ldots, \mathrm{E}_{r}, \mathrm{~F}_{1}, \ldots, \mathrm{F}_{s+1}$ representing a class in $A_{r, s+2}$. We now consider the Hilbert space $\ell^{2}(\mathbb{Z}) \otimes V \otimes \mathbb{C}^{2}$ and again consider the spectral flow via a flux insertion through a unit cell. Namely, we consider the path of Hamiltonians $\mathbf{H}_{\alpha}=\widehat{S}_{\alpha}+\widehat{S}_{\alpha}^{*}$, where

$$
\widehat{S}_{\alpha}=S \otimes F_{s+1} \otimes \frac{1}{2}\left(\begin{array}{cc}
1 & i \\
i & -1
\end{array}\right)+v_{1} v_{0}^{*} \otimes F_{s+1} \otimes \frac{1}{2}\left(\begin{array}{cc}
e^{-i \pi \alpha}-1 & i\left(e^{i \pi \alpha}-1\right) \\
i\left(e^{-i \pi \alpha}-1\right) & -\left(e^{i \pi \alpha}-1\right)
\end{array}\right) .
$$

As in the case of the Kitaev chain, $\mathbf{H}_{\alpha}$ is a finite-rank perturbation of $\mathbf{H}_{0}$ that sends $F_{s+1}$ to $-F_{s+1}$ on the finite dimensional unit cell through which the flux is inserted. As such, we can easily compute that

$$
\operatorname{sf}_{r, s+2}\left(i H_{\bullet}\right)=\operatorname{ind}_{r, s+2}\left(J_{0}, J_{1}\right)=[V] \in A_{r, s+2} \simeq \mathcal{M}_{r, s+1} / \mathcal{M}_{r, s+2} \text {. }
$$

Using this ad hoc construction, we can find a non-trivial $\mathrm{KO}$-valued spectral flow of any degree via a flux insertion in a Kitaev chain.

Example 5.8 (KO-valued spectral flow in symmetry class AII). Here we outline the basic framework for studying $\mathrm{KO}$-valued spectral flow in systems with a fermionic time-reversal symmetry/quaternionic structure. As outlined in Sec. 1, our constructions are most easily illustrated using the framework of Kennedy and 
Zirnbauer [KeZı16]. The relationship between the Kennedy-Zirnbauer framework and the more standard ten-fold way [RSFL1o] is outlined in [KEZI16, AMZ19].

We start with the Hilbert space $\mathcal{V}$ spanned by the fermionic creation operators (e.g. $\mathcal{V}=\ell^{2}(\Lambda)$ for some countable set $\Lambda$ ) and form the Nambu space $\mathcal{W}=\mathcal{V} \oplus \mathcal{V}^{*}$ with real structure $C=\left(\begin{array}{cc}0 & R^{-1} \\ R & 0\end{array}\right)$ given by the Riesz map $R: \mathcal{V} \rightarrow \mathcal{V}^{*}$. We further assume that $\mathcal{V}$ has a quaternionic structure $\mathrm{T}$, an anti-unitary map such that $\mathrm{T}^{2}=-\mathrm{I}_{\mathcal{V}}$. Hamiltonians $\mathbf{H}$ on the Nambu space $\mathcal{W}$ are of Bogoliubov-de Gennes type, $\mathrm{H}=-\mathrm{CHC}$.

The time-reversal and charge symmetry operators $\mathrm{T}$ and $\mathrm{Q}$ are constructed on $\mathcal{W}$ as follows:

$$
\hat{\mathrm{T}}=\left(\begin{array}{cc}
\mathrm{T} & 0 \\
0 & \mathrm{RTR}^{-1}
\end{array}\right), \quad \mathrm{Q}=\left(\begin{array}{cc}
\mathrm{I} \mathcal{V} & 0 \\
0 & -\mathrm{I}_{\mathcal{V}^{*}}
\end{array}\right) .
$$

We can use these operators to construct a $\mathrm{Cl}_{0,2}$ representation on the real Hilbert space $\mathcal{W}_{\mathbb{R}}$ of elements fixed by $\mathrm{C}$. Namely, we take the generators $\mathrm{F}_{1}=\mathrm{C} \uparrow$, $\mathrm{F}_{2}=i \mathrm{C} \uparrow \mathrm{Q}$ which commute with $\mathrm{C}$.

Following [KeZı16], $\mathbf{H}$ is in symmetry class AII if $[\mathrm{H}, \widehat{\mathrm{T}}]=[\mathrm{H}, \mathrm{Q}]=0$. One then sees that $i \mathrm{H}$ anti-commutes with $F_{1}$ and $F_{2}$. Any continuous Fredholm path $\left\{\boldsymbol{H}_{\mathrm{t}}\right\}_{\mathrm{t} \in[0,1]}$ on $\mathcal{W}$ with invertible endpoints and such that $\left[\boldsymbol{H}_{\mathrm{t}}, \widehat{\top}\right]=\left[\boldsymbol{H}_{\mathrm{t}}, \mathrm{Q}\right]=0$ for all $t$ gives a path $\left\{i H_{t}\right\}_{t \in[0,1]}$ in $\mathcal{F}^{0,3}\left(\mathcal{W}_{\mathbb{R}}\right)$ with quaternionic spectral flow

$$
\mathrm{sf}_{0,4}\left(\mathrm{iH}_{\bullet}\right) \in \mathcal{M}_{0,3} / \mathcal{M}_{0,4}, \quad \mathrm{SF}_{0,4}\left(i \mathrm{H}_{\bullet}\right) \in \mathbb{Z}
$$

with $\mathrm{SF}_{0,4}$ measured by a sum of quaternionic Fredholm indices.

We note that any Hamiltonian $\mathbf{H}$ such that $[\mathbf{H}, \widehat{\top}]=[\mathbf{H}, \mathrm{Q}]=0$ and $\mathrm{CHC}=-\mathbf{H}$ must be of the form $\mathbf{H}=\left(\begin{array}{cc}h & 0 \\ 0 & -\mathrm{RhR}^{-1}\end{array}\right)$ with $\mathrm{h}$ a self-adjoint operator on $\mathcal{V}$ with $[h, T]=0$. Considering $\mathcal{V}$ as a real vector space, any eigenvalue of $h$ will have a four-fold degeneracy coming from the quaternionic structure $\{i, j, k\} \sim\{i, T, i T\}$. Given a Fredholm path $h_{\bullet}$ in $\mathcal{V}$ with $h_{t}=h_{t}^{*},\left[h_{t}, T\right]=0$ for all $t$ and such that $h_{0}$ and $h_{1}$ are invertible, we can consider the standard spectral flow SF( $\left.h_{\bullet}\right)$. Passing to the Nambu space $\mathcal{V} \oplus \mathcal{V}^{*}$, because $\sigma\left(h_{t}\right)=\sigma\left(R h_{t} R^{-1}\right)$, the kernel of $\mathbf{H}_{t}$ will be double the dimension of $\operatorname{ker}\left(h_{t}\right)$. This observation along with the doubling of Hilbert spaces gives us that

$$
\frac{1}{4} \mathrm{SF}\left(\mathrm{h}_{\bullet}\right)=\mathrm{SF}_{0,4}(\mathrm{iH}) \in \mathbb{Z}
$$

As such, simple examples of non-trivial quaternionic spectral flow can be written down from a non-trivial and T-symmetric spectral flow of $h_{\bullet}$ in $\mathcal{V}$. We again remark that by taking complex dimension of the kernels, then this quaternionic spectral flow takes values in $2 \mathbb{Z}$.

\section{EXTENSION TO UNBOUNDED OPERATORS}

In applications the operators of interest are often differential operators and hence unbounded. Our approach to $\mathrm{sf}_{\mathrm{r}, \mathrm{s}+2}$ via the Cayley transform extends almost seamlessly to unbounded operators. However, the uniqueness proofs are 
slightly more complicated in the unbounded case than in the bounded case, mainly due to complications in the homotopy theory. Our discussion parallels the complex case that is found in [Leso5].

6.1. Topologies on spaces of unbounded operators. We briefly introduce here the several topologies on unbounded operators, for proofs and further details see [LESO5, Sec. 2]. Let $\mathrm{H}$ be a real Hilbert space. We recall that a densely defined operator $T: \operatorname{dom}(T) \subset \mathrm{H} \rightarrow \mathrm{H}$ is closed if $\operatorname{dom}(T)$ is a complete space with respect to the graph norm

$$
\|v\|_{\mathrm{T}}:=\sqrt{\|v\|^{2}+\|\mathrm{T} v\|^{2}}
$$

We denote by $\mathrm{C}(\mathrm{H})$ we denote the space of densely defined closed operators in $\mathrm{H}$ equipped with the so-called gap topology. It can be described in several equivalent ways. For $T_{1}, T_{2} \in \mathcal{C}(H)$ the gap distance is $d_{G}\left(T_{1}, T_{2}\right)=\left\|P_{1}-P_{2}\right\|$, where $P_{j}$ denotes the orthogonal projection onto the graph of $T_{j}$ in the product space $\mathrm{H} \times \mathrm{H}$. For skew-adjoint $T_{j}$ the gap distance is equivalent to $\left\|\left(T_{1}-I\right)^{-1}-\left(T_{2}-I\right)^{-1}\right\|$.

For skew-adjoint operators one can consider the Riesz transform $\mathrm{F}(\mathrm{T}):=\mathrm{T}(\mathrm{I}-$ $\left.T^{2}\right)^{-1}$. The corresponding Riesz metric $d_{R}\left(T_{1}, T_{2}\right)=\left\|F\left(T_{1}\right)-F\left(T_{2}\right)\right\|$ is stronger than the gap metric.

Finally, we consider operators with a fixed given domain: consider a fixed skew-adjoint operator $\mathrm{D}$ in $\mathrm{H}$ with domain $\mathrm{W} \hookrightarrow \mathrm{H}$. The operator $\mathrm{D}$ serves as reference operator and is part of the structure. Let

$$
\mathcal{B}^{1}(\mathrm{~W}, \mathrm{H}):=\left\{\mathrm{T} \in \mathcal{C}(\mathrm{H}) \mid \mathrm{T}=-\mathrm{T}^{*} \text { and } \operatorname{dom}(\mathrm{T})=\mathrm{W}\right\} .
$$

This space is naturally equipped with the norm given by $\|\mathrm{T}\|_{W \rightarrow H}=\| \mathrm{T}(\mathrm{I}-$ $\left.D^{2}\right)^{-1 / 2} \|$. With $\mathrm{D}$ being any fixed complex structure on $\mathrm{H}$ and $\mathrm{W}=\mathrm{H}$ we obtain $\mathcal{B}^{1}(H, H)=\mathcal{B}^{1}(H)$ the bounded skew-adjoint operators on $H$ as a special case.

Remark 6.1. A word of warning: one might be tempted to "identify" $\mathcal{B}^{1}(W, H)$ with the skew-adjoint operators in $\mathrm{H}$ by sending

$$
\mathcal{B}^{1}(\mathrm{~W}, \mathrm{H}) \ni \mathrm{T} \rightarrow\left(\mathrm{I}-\mathrm{D}^{2}\right)^{-1 / 4} \mathrm{~T}\left(\mathrm{I}-\mathrm{D}^{2}\right)^{-1 / 4}=: \mathrm{B}(\mathrm{T}) .
$$

Using a bit of interpolation theory (cf. [Leso5, Appendix]) one sees that indeed $\mathrm{B}(\mathrm{T})$ is a skew-adjoint bounded operator, and the map $\mathrm{T} \mapsto \mathrm{B}(\mathrm{T})$ is injective. However, it is in general not surjective. Namely, interpolation theory shows that for an operator $S \in \mathcal{B}^{1}(H)$ to be in the range of $B$ it is necessary that

$$
\left(I-D^{2}\right)^{\alpha} S\left(I-D^{2}\right)^{-\alpha}
$$

is bounded on $\mathrm{H}$ for $-1 / 2 \leq \alpha \leq 1 / 2$. In the interesting case of unbounded $\mathrm{D}$, this is a restriction which is not satisfied by all operators in $\mathcal{B}^{1}(H)$.

If $\mathrm{H}$ is a $\mathrm{Cl}_{r, s}-$ Hilbert space then we denote by $\mathrm{C}^{\mathrm{r}, \mathrm{s}+1}, \mathcal{B}^{\mathrm{r}, \mathrm{s}+1}, \ldots$ the corresponding spaces of skew-adjoint operators which anti-commute with the Clifford generators $E_{1}, \ldots, E_{r}, F_{1}, \ldots, F_{s}$, cf. Sec. 2.1. In the case of operators with a fixed domain this requires, of course, that $\mathrm{D}$ itself is $\mathrm{C}_{r, s}$-anti-linear and hence the inclusion $\mathrm{W} \hookrightarrow \mathrm{H}$ is an inclusion of $\mathrm{Cl}_{\mathrm{r}, \mathrm{s}}-$ Hilbert spaces. Similarly, $\mathrm{eF}^{\mathrm{r}, \mathrm{s}+1}(\mathrm{H})$ 
and $\mathcal{F}^{\mathrm{r}, \mathrm{s}+1}(\mathrm{~W}, \mathrm{H})$ denote the corresponding spaces of skew-adjoint Fredholm operators.

The relations between the metric spaces of unbounded operators are summarized by noting that that there are natural continuous (inclusion) maps [LESO5, Prop. 2.2]

$$
\mathcal{B}^{r, s+1}(W, H) \hookrightarrow\left(\mathcal{C}^{r, s+1}(H), d_{R}\right) \stackrel{\text { id }}{\longrightarrow}\left(\mathcal{C}^{r, s+1}(H), d_{G}\right),
$$

where $d_{R}, d_{G}$ denote the Riesz and gap distance resp.

6.2. Spectral flow for families of unbounded operators. With the preparations

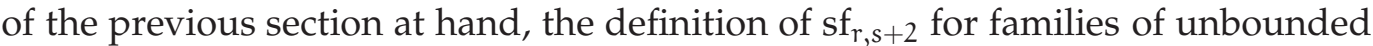
skew-adjoint Fredholm operators is straightforward. Let us consider a $\mathrm{Cl}_{r, s}-$ Hilbert space $H$ and a gap continuous family $T_{0}:[0,1] \rightarrow \mathcal{C F}^{r, s+1}(H)$ of unbounded skew-adjoint Fredholm operators with invertible endpoints. As remarked above, the gap continuity implies (and is in fact equivalent to) the continuity of the resolvent maps $t \mapsto\left(T_{t} \pm I\right)^{-1}$. Hence, the Cayley transform (Definition 5.1)

$$
\mathrm{t} \mapsto \Phi\left(\mathrm{T}_{\mathrm{t}}\right)=-\mathrm{F}_{\mathrm{s}}\left(\mathrm{I}+\mathrm{T}_{\mathrm{t}} \mathrm{F}_{\mathrm{s}}\right)\left(\mathrm{I}-\mathrm{T}_{\mathrm{t}} \mathrm{F}_{\mathrm{s}}\right)^{-1}
$$

is a continuous path $([0,1],\{0,1\}) \rightarrow\left(\widetilde{\Omega}_{r, s}, \widetilde{\Omega}_{r, s, *}\right)$ in $\widetilde{\Omega}_{r, s}$ with endpoints in the contractible (see Lemma 3.3) neighbourhood $\widetilde{\Omega}_{r, s, *}$ of $F_{s}$. One is now in the situation of Def. 5.1 and therefore proceeding as in loc. cit. one obtains an element $\mathrm{sf}_{\mathrm{r}, s+2}\left(\mathrm{~T}_{\bullet}\right) \in \mathrm{A}_{\mathrm{r}, \mathrm{s}+2}$ and $\mathrm{SF}_{\mathrm{r}, s+2}:=\tau_{\mathrm{r}, s+2}\left(\mathrm{sf}_{\mathrm{r}, s+2}\left(\mathrm{~T}_{\bullet}\right)\right) \in \mathrm{KO}_{s+2-\mathrm{r}}(\mathbb{R})$, where $\tau$ was defined in Eq. (2.17).

The reader might notice that alternatively one could first have introduced a spectral flow invariant of paths in $([0,1],\{0,1\}) \rightarrow\left(\widetilde{\Omega}_{r, s}, \widetilde{\Omega}_{r, s, *}\right)$ as the basic object. The spectral flow of paths of bounded and unbounded skew-adjoint Fredholm operators are then just obtained by pullback via the map $\Phi$.

From the homotopy theory of $\widetilde{\Omega}_{r, s}$ it is clear that the $s_{r, s+2}$ for gap continuous families of skew-adjoint Fredholm operators satisfies Homotopy, Path additivity, Stability, Normalization, Constancy, and Direct sum in the sense of Remark 5.2.

In view of the inclusions Eq. (6.1) the $\mathrm{sf}_{\mathrm{r}, \mathrm{s}+2}$ for Riesz continuous families of skew-adjoint Fredholm operators (resp. such families in the space $\mathcal{F}^{r}, s+1(W, H)$ ) is just obtained by taking the $\mathrm{sf}_{\mathrm{r}, \mathrm{s}+2}$ after sending the family to $\mathrm{eFF}^{\mathrm{r}, \mathrm{s}+1}$ via the natural maps Eq. (6.1).

6.3. Two technical results. In the unbounded case we will need adaptations of the two technical results [Leso5, Assertion 1 and 2 in Sec. 5]. The first statement is an improvement of the results in Lemma 5.3.

Lemma 6.2. Let $\mathrm{H}$ be a $\mathrm{Cl}_{\mathrm{r}, \mathrm{s}}$-Hilbert space and let $\mathrm{T}_{\bullet}:[0,1] \ni \mathrm{t} \mapsto \mathrm{CF}^{\mathrm{r}, \mathrm{s}+1}(\mathrm{H})$ be a continuous path with invertible endpoints. Then there exists a subdivision $0=t_{0}<$ $\mathrm{t}_{1} \cdots<\mathrm{t}_{\mathrm{n}}=1$ of the interval $[0,1]$ and a continuous path $\widetilde{\mathrm{T}}:[0,1] \rightarrow \mathcal{C F}^{\mathrm{r}, \mathrm{s}+1}(\mathrm{H})$ with the following properties:

(1) $\widetilde{T}_{t}$ is a finite rank perturbation of $T_{t}$ and it has the same endpoints as $T_{0}$, i.e. $\widetilde{\mathrm{T}}_{0}=\mathrm{T}_{0}, \widetilde{\mathrm{T}}_{1}=\mathrm{T}_{1}$. 
(2) $\widetilde{T}_{t_{j}}$ is invertible, $j=0, \ldots, n$. The value of $T_{t_{j}}$ may even be prescribed as in Lemma 5.3 .

(3) There exist $\varepsilon_{j}>0, j=1, \ldots, n$ such that for all $t \in\left[t_{j-1}, t_{j}\right]$ we have $\varepsilon_{j} \notin$ $\operatorname{spec}\left|\mathrm{T}_{\mathrm{t}_{\mathrm{j}}}\right|$ and $\operatorname{spec}_{\mathrm{ess}}\left(\left|\mathrm{T}_{\mathrm{t}_{\mathrm{j}}}\right|\right) \cap\left[0, \varepsilon_{\mathrm{j}}\right]=\emptyset$.

The properties (1)-(2) imply that $\widetilde{T}_{0}$ is (straight-line) homotopic to $T_{0}$ and hence both paths have the same $\mathrm{sf}_{\mathrm{r}, \mathrm{s}+1}$ spectral flow.

The result holds verbatim also for Riesz continuous paths in $\mathrm{CF}^{\mathrm{r}, \mathrm{s}+1}(\mathrm{H})$ as well as for continuous paths into $\mathcal{F}^{\mathrm{r}, \mathrm{s}+1}(\mathrm{~W}, \mathrm{H})$.

Proof. To avoid repetitive statements a like 'resp. for Riesz continuous resp for paths in $\mathcal{F}^{r, s+1}(\mathrm{~W}, \mathrm{H})^{\prime}$ we present the proof for gap continuous paths and leave the (obvious) modifications for the other two cases to the reader.

Clearly, since $T_{t}$ is Fredholm and by compactness there is a subdivision $0=t_{0}<$ $\mathrm{t}_{1}<\ldots<\mathrm{t}_{\mathrm{n}}=1$ and $\varepsilon_{j}>0$ such that $\varepsilon_{j} \notin \operatorname{spec}|\mathrm{T}(\mathrm{t})|$ and $\operatorname{spec}_{\text {ess }}(|\mathrm{T}(\mathrm{t})|) \cap\left[0, \varepsilon_{j}\right]=$ $\emptyset$. The problem is that $T\left(t_{j}\right)$ need not be invertible. However, an inspection of the proof of Lemma 5.3 shows, that it holds for paths in $\mathrm{eF}^{\mathrm{r}, \mathrm{s}+1}$ as well. More precisely, the proof shows that there are finite rank $\mathrm{Cl}_{\mathrm{r}, \mathrm{s}}$-antilinear skew-adjoint operators $R_{j}$ (and the same choices as in Lemma 5.3 are possible) with $\left.R_{j}\right|_{k e r T_{t_{j}}} \in$ $g^{r, s+1}\left(\operatorname{ker} T_{t_{j}}\right)$ and $\left.R_{j}\right|_{\text {ker } T_{t_{j}}^{\perp}}=0$. With the notation of the proof of Lemma 5.3 we see that for $\lambda>0$ the operator family $\widetilde{T}_{t}(\lambda):=T_{t}+\lambda \cdot \sum_{j=1}^{n-1} \varphi_{j}(t) R_{j}$ satisfies (1) and (2) for all $\lambda>0$. By compactness, there is a $\delta>0$ such that for all $0<\lambda \leq \delta$ the family $\widetilde{T}_{\bullet}(\lambda)$ satisfies ( 3 ) as well.

Lemma 6.3. Let $\mathrm{H}$ be a $\mathrm{Cl}_{\mathrm{r}, \mathrm{s}}$-Hilbert space and let $\mathrm{T}_{\bullet}:[0,1] \ni \mathrm{t} \mapsto \operatorname{efF}^{\mathrm{r}, \mathrm{s}+1}(\mathrm{H})$ be a gap continuous path with invertible endpoints. Furthermore, assume that (3) of the previous Lemma is fullfilled for $T_{0}$. More precisely, assume that there exists an $\varepsilon>0$ such that for all $\mathrm{t} \in[0,1]$ we have $\varepsilon \notin \operatorname{spec}\left|\mathrm{T}_{\mathrm{t}}\right|$ and $\operatorname{spec}_{\mathrm{ess}}\left(\left|\mathrm{T}_{\mathrm{t}}\right|\right) \cap[0, \varepsilon]=\emptyset$.

Let $\mathrm{E}(\mathrm{t})=1_{[0, \varepsilon]}\left(\left|\mathrm{T}_{\mathrm{t}}\right|\right)$ be the finite-rank spectral projection onto the eigenspaces with eigenvalues $\lambda \leq \varepsilon$ of $\left|T_{t}\right|$.

Then there is a continuous family $\mathrm{U}:[0,1] \rightarrow \mathcal{B}(\mathrm{H})$ of unitary operators commuting with the $\mathrm{Cl}_{r, s}$-action such that $\widetilde{\mathrm{T}}_{\mathrm{t}}=\mathrm{U}(\mathrm{t})^{*} \mathrm{~T}_{\mathrm{t}} \mathrm{U}(\mathrm{t})$ has the following properties:

(1) $\widetilde{T}_{0}:[0,1] \ni \rightarrow \mathcal{C F}^{\mathrm{r}, s+1}$ with invertible endpoints.

(2) $\widetilde{T}_{0}$ is homotopic to $T_{0}$ within paths in $\mathrm{eF}^{\mathrm{r}, \mathrm{s}+1}$ with invertible endpoints.

(3) $\widetilde{\mathrm{T}}_{\mathrm{t}}$ commutes with $\mathrm{E}(0)$ and $\left.\widetilde{\mathrm{T}}_{\mathrm{t}}\right|_{\mathrm{ran} \mathrm{E}(0)}$ is invertible.

As a consequence $\mathrm{T}_{\bullet}$ is homotopic within paths in $\mathrm{eF}^{\mathrm{r}, \mathrm{s}+1}$ with invertible endpoints to a path $\mathrm{T}_{0}^{1}$ commuting with $\mathrm{E}(0)$ and such that $\left.\mathrm{T}_{\bullet}^{1}\right|_{\mathrm{ker} \mathrm{E}(\mathrm{O})}$ is constant and invertible.

The result holds verbatim also for Riesz continuous paths in $\mathrm{CF}^{\mathrm{r}, \mathrm{s}+1}(\mathrm{H})$ as well as for continuous paths into $\mathcal{F}^{\mathrm{r}, \mathrm{s}+1}(\mathrm{~W}, \mathrm{H})$.

Proof. Note that

$$
E(t)=\frac{1}{2 \pi i} \oint\left(-T_{t}^{2}-z\right)^{-1} d z,
$$

where the integral is along a contour encircling the interval $\left[0, \varepsilon^{2}\right]$ counterclockwise such that no other spectral points are encircled. This is possible by the 
spectral gap assumptions. This formula shows that $t \mapsto E(t)$ is continuous, in view of Eq. (6.1) a fortiori if $T_{\bullet}$ is Riesz continuous or fixed domain continuous. Furthermore, $\mathrm{E}(\mathrm{t})$ commutes with the $\mathrm{C}_{\mathrm{r}, \mathrm{s}}$-action. The construction in the proof of Prop. 3.2 and in [BLA98, Prop. 4.3.3] shows that there is a continuous family of $\mathrm{Cl}_{r, s}$-linear unitaries $\mathrm{U}:[0,1] \rightarrow \mathcal{B}(\mathrm{H}), \mathrm{U}(0)=\mathrm{I}$ such that $\mathrm{E}(\mathrm{t})=\mathrm{U}(\mathrm{t}) \mathrm{E}(0) \mathrm{U}(\mathrm{t})^{*}$. Now put $\widetilde{T}_{t}:=U(t) * T_{t} U(t)$.

Since $E(t)$ and $U(t)$ are $C \ell_{r, s}$-linear, the rest of the proof is now identical to that in [LESO5, Assertion 2 in Sec. 5].

6.4. The 'local formula' for SF in the unbounded case and consequences. Next we discuss the analogue of Theorem 5.4. For this we will need the two technical Lemmas in Sec. 6.3. For Theorem 5.4 however, it is essential that the family $t \mapsto \pi\left(J_{t}\right) \in \mathcal{Q}(H)$ depends continuously on $t$ where $J_{t}$ is essentially the phase $T_{t}\left|T_{t}\right|^{-1}$. For this to behave continuously, gap continuity is not enough. Therefore, the analogue of Theorem 5.4 can only be proved for Riesz continuous families of skew-adjoint Fredholm operators.

Theorem 6.4. Let $\mathrm{H}$ be a $\mathrm{Cl}_{\mathrm{r}, \mathrm{s}}$-Hilbert space and let $[0,1] \ni \mathrm{t} \mapsto \mathrm{T}_{\mathrm{t}} \in \mathrm{CF}^{\mathrm{r}, \mathrm{s}+1}(\mathrm{H})$ be a Riesz continuous path with $\mathrm{T}_{0}, \mathrm{~T}_{1}$ being invertible. Let $\mathrm{J}_{\mathrm{t}}$ be complex structures obtained by complementing the phase $\mathrm{T}_{\mathrm{t}}\left|\mathrm{T}_{\mathrm{t}}\right|^{-1}$ on $\mathrm{ker} \mathrm{T}_{\mathrm{t}}^{\perp}$ by an arbitrary complex structure in $\mathrm{J}^{\mathrm{r}, \mathrm{s}+1}\left(\operatorname{ker} \mathrm{T}_{\mathrm{t}}\right), c f$. Eq. (5.2).

Then $\mathrm{t} \mapsto \pi\left(\mathrm{J}_{\mathrm{t}}\right) \in \mathcal{Q}(\mathrm{H})$ is continuous. For a fine enough ${ }^{10}$ partition $0=\mathrm{t}_{0}<\mathrm{t}_{1}<$ $\cdots<t_{n}=1$ of the interval $[0,1]$ we then have the formula

$$
\mathrm{SF}_{r, s+2}\left(\mathrm{~T}_{\bullet}\right)=\sum_{j=1}^{n} \operatorname{Ind}_{r, s+2}\left(J_{t_{j-1}}, J_{t_{j}}\right) .
$$

Proof. The continuity of $t \mapsto \pi\left(J_{t}\right) \in \mathcal{Q}(H)$ follows as in [Leso5, Lemma 3.3]. By the path additivity and homotopy invariance of the spectral flow and by Lemma 6.2, it suffices to prove the Theorem for a family $T_{t}$ satisfying the spectral gap assumptions of Lemma 6.3. We can further assume that $E(t)-E(0)$ and $U(t)-U(0)$ and the Riesz distance $d_{R}\left(T_{t}, T_{0}\right)$ are small enough, and finally

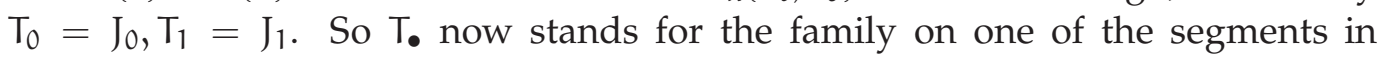
Lemma 6.2 and it remains to show that $\mathrm{SF}_{\mathrm{r}, \mathrm{s}+2}\left(\mathrm{~T}_{\bullet}\right)=\operatorname{Ind}_{\mathrm{r}, \mathrm{s}+2}\left(\mathrm{~J}_{0}, \mathrm{~J}_{1}\right)$. With respect to the decomposition $\mathrm{H}=\operatorname{ker} \mathrm{E}(0) \oplus \operatorname{ran} \mathrm{E}(0)$ we have

$$
\mathrm{U}(\mathrm{t})^{*} \mathrm{~T}_{\mathrm{t}} \mathrm{U}(\mathrm{t})=\mathrm{S}_{\mathrm{t}} \oplus \mathrm{R}_{\mathrm{t}}
$$

\footnotetext{
${ }^{10}$ The phrase 'fine enough' can be quantified by working through the constructions in the proof.
} 
with an invertible family $S_{\text {. }}$ and a finite rank family $R_{\bullet}$. Again by Homotopy and applying Theorem 5.4 and Prop. 4.7 to the finite-rank family $R_{\text {. we have }}$

$$
\begin{aligned}
\mathrm{SF}_{\mathrm{r}, \mathrm{s}+2}\left(\mathrm{~T}_{\bullet}\right) & =\mathrm{SF}_{\mathrm{r}, \mathrm{s}+2}\left(\mathrm{U}(\bullet)^{*} \mathrm{~T}_{\bullet} \mathrm{U}(\bullet)\right) \\
& =\mathrm{SF}_{r, s+2}\left(\mathrm{R}_{\bullet}\right) \\
& =\operatorname{Ind}_{\mathrm{r}, \mathrm{s}+2}\left(\left.\mathrm{U}(0)^{*} \mathrm{~J}_{0}\right|_{\operatorname{ran~E}(0)} \mathrm{U}(0),\left.\mathrm{U}(1)^{*} \mathrm{~J}_{1} \mathrm{U}(1)\right|_{\operatorname{ran} \mathrm{E}(0)}\right) \\
& =\operatorname{Ind}_{\mathrm{r}, \mathrm{s}+2}\left(\mathrm{U}(0)^{*} \mathrm{~J}_{0} \mathrm{U}(0), \mathrm{U}(1)^{*} \mathrm{~J}_{1} \mathrm{U}(1)\right) \\
& =\operatorname{Ind}_{r, s+2}\left(\mathrm{~J}_{0}, \mathrm{U}(0) \mathrm{U}(1)^{*} \mathrm{~J}_{1} \mathrm{U}(1) \mathrm{U}(0)^{*}\right) \\
& =\operatorname{Ind}_{\mathrm{r}, \mathrm{s}+2}\left(\mathrm{~J}_{0}, \mathrm{~J}_{1}\right)
\end{aligned}
$$

From line 3 to line 4 it was used that $S_{0}, S_{1}$ is an invertible pair since they are close enough in the Riesz distance. For the last equality, one may invoke the homotopy $\mathrm{U}(0) \mathrm{U}(\mathrm{s})^{*} \mathrm{~J}_{1} \mathrm{U}(\mathrm{s}) \mathrm{U}(0)^{*}, 0 \leq \mathrm{s} \leq 1$, again using the smallness assumptions which imply that this is a valid homotopy of Fredholm pairs.

Theorem 6.5 (cf. [Leso5, Theorem 3.6]). Let $\mathrm{H}$ be a $\mathrm{C}_{\mathrm{r}, \mathrm{s}}-$ Hilbert space and let $[0,1] \ni$ $\mathrm{t} \mapsto \mathrm{T}_{\mathrm{t}} \in \mathrm{eF}^{\mathrm{r}, \mathrm{s}+1}(\mathrm{H})$ be a Riesz continuous path with $\mathrm{T}_{0}, \mathrm{~T}_{1}$ being invertible. Assume furthermore, that the domain of $\mathrm{T}_{\mathrm{t}}$ does not depend on $\mathrm{t}$ and that for each $\mathrm{t} \in[0,1]$ the difference $T_{t}-T_{0}$ is relatively $T_{0}$-compact, i.e. $\left(T_{t}-T_{0}\right)\left(T_{0}+I\right)^{-1}$ is compact. Then the pair $\left(\mathrm{T}_{0}\left|\mathrm{~T}_{0}\right|^{-1}, \mathrm{~T}_{1}\left|\mathrm{~T}_{1}\right|^{-1}\right)$ consisting of the phases of the endpoints is a Fredholm pair of complex structures in $\mathrm{g}^{\mathrm{r}, \mathrm{s}+1}(\mathrm{H})$ and we have the index formula

$$
\operatorname{sf}_{r, s+2}\left(T_{\bullet}\right)=\operatorname{ind}_{r, s+2}\left(T_{0}\left|T_{0}\right|^{-1}, T_{1}\left|T_{1}\right|^{-1}\right) .
$$

Proof. It follows from [Leso5, Prop. 3.4] that under the assumptions of the Theorem the differences of the Riesz transforms $F\left(T_{t}\right)-F\left(T_{0}\right)$ is a compact operator for all $t \in[0,1]$. Similarly, the differences of the 'phases' $J_{t}-J_{0}$ are compact as well. Now the claim follows by applying Prop. 4.7 to the formula in Theorem 6.4.

Thus, for a path of relatively compact operators the $\mathrm{SF}_{\mathrm{r}, \mathrm{s}+2}$ depends only on the endpoints. This applies in particular to the finite dimensional case which we single out here.

Corollary 6.6. Let $\mathrm{H}$ be a finite dimensional $\mathrm{C}_{\mathrm{r}, \mathrm{s}}$-module and let $[0,1] \ni \mathrm{t} \mapsto \mathrm{T}_{\mathrm{t}} \in$ $\mathcal{B}^{r, s+1}(\mathrm{H})$ be a continuous family of skew-adjoint matrices anti-commuting with the Clifford generators. Then $\mathrm{sf}_{\mathrm{r}, \mathrm{s}+2}\left(\mathrm{~T}_{\bullet}\right)=\mathrm{ind}_{\mathrm{s}, \mathrm{r}+2}\left(\mathrm{~T}_{0}\left|\mathrm{~T}_{0}\right|^{-1}, \mathrm{~T}_{1}\left|\mathrm{~T}_{1}\right|^{-1}\right)$.

\section{UNIQUENESS OF THE KO-VALUED SPECTRAL FLOW}

In this section we give an 'axiomatic' characterization of $\mathrm{KO}$-valued spectral flow. Our approach is not exactly the same as in the complex case as described in [LESO5] because we have to start with the finite dimensional case. As a result, stability must play a role in the passage to infinite dimensions.

7.1. Uniqueness in the finite dimensional case. We introduce some notation. For a finite dimensional $\mathrm{Cl}_{\mathrm{r}, \mathrm{s}}$-module $\mathrm{H}$ we denote by $\mathcal{A}^{\mathrm{r}, \mathrm{s}+1}(\mathrm{H})$ the set of continuous paths $\gamma:[0,1] \rightarrow \mathcal{B}^{r, s+1}(\mathrm{H})$ with $\gamma(0), \gamma(1)$ being invertible. 
Theorem 7.1. Suppose we are given, for each finite dimensional $\mathrm{C}_{\mathrm{r}, \mathrm{s}}$-module $\mathrm{V}$, a map $\mu_{V}: \mathcal{A}^{r, s+1}(\mathrm{~V}) \rightarrow A_{r, s+2}$ satisfying Homotopy, Path additivity, Stability, and Normalization in the sense of Remark 5.2. Then $\mu_{\mathrm{V}}=\mathrm{sf}_{\mathrm{r}, \mathrm{s}+2}$.

More precisely, Normalization means the following: if $\mathrm{V}$ is a module as in Eq. (5.1) (resp. Sec. 4.3) then $\mu_{V}\left(t \mapsto(1-2 t) F_{s+1}\right)=[V]$.

Proof. Suppose first, we are given two paths $\gamma_{1}, \gamma_{2} \in \mathcal{A}^{\mathrm{r}, \mathrm{s}+1}(\mathrm{~V})$ with the same endpoints. Then the straight line homotopy $u \mapsto(1-u) \gamma_{1}+\mathfrak{u} \gamma_{2}$ stays within $\mathcal{A}^{r, s+1}(V)$, hence $\mu_{V}\left(\gamma_{1}\right)=\mu_{V}\left(\gamma_{2}\right)$. Thus for $\gamma \in \mathcal{A}^{r, s+1}(V)$ the value $\mu_{V}(\gamma)$ depends only on the endpoints of $\gamma$. In particular, $\mu_{V}(\gamma)=\mu_{V}(t \mapsto(1-t) \gamma(0)+t \gamma(1))$.

If $h_{0}(u), h_{1}(u)$ are paths of invertible elements in $\mathcal{B}^{r, s+1}(V)$ with $h_{0}(0)=\gamma(0)$ and $h_{1}=\gamma(1)$, then $H(u, t):=(1-t) h_{0}(u)+t h_{1}(u)$ is a homotopy with invertible endpoints from the straight line path from $\gamma(0)$ to $\gamma(1)$ to the straight line path from $h_{0}(1)$ to $h_{1}(1)$.

Together with Stability this shows that $\mu_{V}(\gamma)$ depends only on the stable homotopy classes of the endpoints of the paths. Since any invertible $T \in \mathcal{B}^{r, s+1}(V)$ is homotopic to its phase $\mathrm{T}|\mathrm{T}|^{-1} \in \mathrm{J}^{\mathrm{r}, s+1}(\mathrm{~V})$, it suffices to consider pairs of complex structures. By Appendix A.2 we know that in each stable path component there is a standard pair as in Sec. 4.3. Hence by Normalization (and Stability) the claim follows.

7.2. Uniqueness in the fixed domain unbounded case (includes bounded case). Here we discuss uniqueness for families in $\mathcal{F}^{r, s+1}(W, H)$. This contains the case $\mathrm{W}=\mathrm{H}$ and hence the bounded case as a special case. The obvious extension to gap or Riesz continuous families is left to the reader, but see [LESO5, Sec. 5].

So fix a $\mathrm{C}_{r, s}-$ Hilbert space and a skew-adjoint operator $\mathrm{D} \in \mathrm{CF}^{\mathrm{r}, s+1}(\mathrm{H})$ with domain $W$. By $\mathcal{A}^{r, s+1}(W, H)$ we denote the set of continuous paths $\gamma:[0,1] \rightarrow$ $\mathcal{F}^{r, s+1}(\mathrm{~W}, \mathrm{H})$ with invertible endpoints. A pair $(\widetilde{W}, \widetilde{H})$ is called $W$-admissible if there is a finite dimensional $C \ell_{r, s+1}-$ module $V$ such that $\widetilde{W}=W \oplus V, \widetilde{\mathrm{H}}=\mathrm{H} \oplus \mathrm{V}$ as $\mathrm{Cl}_{r, s}-$ Hilbert spaces. Hence $\widetilde{W} \hookrightarrow \widetilde{\mathrm{H}}$ sits naturally in $\widetilde{\mathrm{H}}$ as a dense subspace. $\widetilde{W}$ is the domain, e.g., of the skew-adjoint operator $\left.D \oplus F_{s+1}\right|_{V}$.

Theorem 7.2. Suppose that for each $\mathrm{W}$-admissible pair $(\widetilde{\mathrm{W}}, \widetilde{\mathrm{H}})$ we are given a map $\mu_{\widetilde{W}}: \mathcal{A}^{r, s+1}(\widetilde{W}, \widetilde{\mathrm{H}}) \rightarrow \mathrm{A}_{\mathrm{r}, \mathrm{s}+2}$ satisfying Homotopy, Path additivity, and Stability in the sense of Remark 5.2. Furthermore, assume that $\mu$ satisfies the following variant of Normalization:

Let $\mathrm{T} \in \mathcal{F}^{\mathrm{r}, \mathrm{s}+1}(\mathrm{~W}, \mathrm{H})$ be an invertible operator. Furthermore, let $\mathrm{V}$ be a finite dimensional $\mathrm{C}_{r, s+1}$-module. Consider the path

$$
\gamma(\mathrm{t}):=\mathrm{T} \oplus(1-2 \mathrm{t}) \mathrm{F}_{\mathrm{s}+1} \in \mathcal{F}^{\mathrm{r}, \mathrm{s}+1}(\mathrm{~W} \oplus \mathrm{V}, \mathrm{H} \oplus \mathrm{V}) .
$$

Then $\mu_{\mathrm{W} \oplus \mathrm{V}}(\gamma)=[\mathrm{V}] \in \mathrm{A}_{\mathrm{r}, \mathrm{s}+2}$.

Then $\mu_{\widetilde{W}}=\mathrm{SF}_{\mathrm{r}, \mathrm{s}+2}$ for all admissible pairs $(\widetilde{\mathrm{W}}, \widetilde{\mathrm{H}})$. 
Proof. Let $\mathrm{T}_{\text {• }}$ be given. Apply Lemma 6.2 and let $\widetilde{T}_{\bullet}$ be the corresponding finiterank modification of $T_{\bullet}$. By Homotopy and Path additivity we have

$$
\mu_{W}(T)=\mu_{W}(\widetilde{T})=\sum_{j=1}^{n} \mu_{W}\left(\left.\widetilde{T}\right|_{\left[t_{j-1}, t_{j}\right]}\right) .
$$

We look at one of the $\left.\widetilde{T}\right|_{\left[t_{j-1}, t_{j}\right]}$ and write again $T$ for it and parametrize it over $[0,1]$. As a result of this exercise we may now assume that there is a global spectral gap $\varepsilon>0$ such that $T$ satisfies the prerequisites of Lemma 6.3. By homotopy

$$
\mu_{W}\left(T_{\bullet}\right)=\mu_{W}\left(R_{t} \oplus S\right),
$$

where $S$ is constant and invertible on $\operatorname{ker} E(0)$ and $R_{t}$ is finite-rank on ran $E(0)$.

There is a slight difficulty here, as $\operatorname{ker} E(0)$ is only a subspace of $H$ (resp. W). Since $R_{0}$ is also invertible on $\operatorname{ran} E(0)$ we put $\widetilde{W}:=\operatorname{ran} E(0) \oplus W$. Then by Stability (and Homotopy to switch the roles of the two copies of $\operatorname{ran} E(0)$ )

$$
\mu_{W}\left(T_{\bullet}\right)=\mu_{\widetilde{W}}\left(R_{t} \oplus\left(R_{0} \oplus S\right)\right) .
$$

By Normalization and the finite dimensional case, which has been proved, the latter equals $\operatorname{sf}_{r, s+2}\left(T_{\bullet}\right)$ and we are done.

\section{The Robbin-Salamon Theorem for KO-valued spectral flow}

The axiomatic characterization of the $\mathrm{KO}$-valued spectral flow in the previous section allows a short proof of our main objective. Namely we now prove the 'spectral flow $=$ Fredholm index' result alluded to in the introduction.

8.1. Standing assumptions. Following [RoSA95, (A1)-(A3)], [KALE13, Sec. 8] we need to introduce quite a bit of notation here. We continue to work in the framework of Fredholm operators with a fixed domain. So let $\mathrm{H}$ be a $\mathrm{C} \ell_{r, s}-$ Hilbert space $\mathrm{H}$ and let $\mathrm{D} \in \mathrm{CF}^{\mathrm{r}, \mathrm{s}+1}(\mathrm{H})$ be a skew-adjoint Fredholm operator with compact resolvent. The latter is equivalent to the saying that the domain $W:=\operatorname{dom}(D)$ with its natural graph norm is compactly embedded into $\mathrm{H}$.

Furthermore, consider a one-parameter family $A(\cdot): \mathbb{R} \mapsto \mathcal{F}^{r, s+1}(W, H)$ of skewadjoint Fredholm operators such that the following conditions are satisfied:

(A 1) The map $A: \mathbb{R} \rightarrow \mathcal{B}^{r, s+1}(W, H)$ is weakly differentiable. This means that for all $\xi \in W$ and all $\eta \in H$ the map $t \mapsto\left\langle A_{1}(t) \xi, \eta\right\rangle$ is differentiable. Furthermore, we suppose that the weak derivative $A^{\prime}(t): W \rightarrow H$ is bounded for each $t \in \mathbb{R}$ and that the supremum $\sup _{t \in \mathbb{R}}\left\|A^{\prime}(t)\right\|_{W \rightarrow H}=: K<\infty$ is finite.

(A 2) The domain $\operatorname{dom}(A(t))=W$ is independent of $t$ and equals $W$. Moreover, there exist constants $C_{1}, C_{2}>0$ such that

$$
\mathrm{C}_{1}\|\xi\|_{W} \leq\|\xi\|_{A(t)} \leq \mathrm{C}_{2}\|\xi\|_{W}
$$

for all $\xi \in W$ and all $t \in \mathbb{R}$. In other words, the graph norms are uniformly equivalent to the norm $\|\cdot\|_{W}$ of $W$.

(A 3) There exists $R, c>0$ such that for all $t \in \mathbb{R}$ with $|t| \geq R$ we have $|A(t)| \geq c \cdot I$, i.e. $A(t)$ is invertible and uniformly bounded below. 
(A 1) and (A 2) are the same as in [RoSA95], while (A 3) is slightly more general than [RoSA95, (A3)], see [KaLE13, Sec. 8].

If $A(\cdot)$ satisfies (A 1 )-(A 3 ) then we let $\operatorname{sf}_{r, s+2}(A(\cdot))$ be the $s f_{r, s+2}$ of the path $[-R, R] \ni t \mapsto A(t)$. We leave the straightforward verification that this is welldefined to the reader. The condition (A 1 ) implies continuity as a map $\mathbb{R} \rightarrow$ $\mathcal{B}^{r, s+1}(\mathrm{~W}, \mathrm{H})$. Furthermore, (A 1$)$ can be relaxed to the assumption that $A(\cdot)$ is continuous and piecewise weakly continuously differentiable. This has the benefit that given $A(t)_{a \leq t \leq b}$ satisfying the finite interval analogues of (A 1$)-(A$ 3) with $A(a), A(b)$ invertible, the family

$$
\widetilde{A}(t):= \begin{cases}A(a), & t \leq a, \\ A(t), & a \leq t \leq b, \\ A(b), & t \geq b,\end{cases}
$$

satisfies the modified axiom (A 1 ) and the axioms (A 2), (A 3).

8.2. The Robbin-Salamon Theorem for $C \ell_{r, s}$-antilinear skew-adjoint Fredholm operators. After these preparations, we are ready to formulate the main result of this section:

Theorem 8.1. Let $\mathrm{A}(\cdot)$ be a family of unbounded skew-adjoint Fredholm operators in $\mathrm{eF}^{\mathrm{r}, \mathrm{s}+1}(\mathrm{~W}, \mathrm{H})$ satisfying the axioms (A 1)-(A 3) above. Let

$$
\begin{aligned}
\mathrm{D} & =A(t) \otimes \omega_{1,1}-\frac{d}{d t} \otimes K_{1} \\
& =\left(\begin{array}{cc}
-\frac{d}{d t} & -A(t) \\
-A(t) & \frac{d}{d t}
\end{array}\right) .
\end{aligned}
$$

Then $\mathrm{D}$ is an essentially skew-adjoint Fredholm operator in $\mathrm{CF}^{\mathrm{r}, \mathrm{s}+2}\left(\mathrm{~L}^{2}\left(\mathbb{R}, \mathrm{H} \otimes \mathbb{R}^{2}\right)\right)$ where the Clifford generators are given by, cf. Prop. 2.3, $\widetilde{\mathrm{E}}_{j}:=\mathrm{E}_{\mathrm{j}} \otimes \omega_{1,1}, j=1, \ldots, r, \widetilde{\mathrm{F}}_{k}:=$ $\mathrm{F}_{\mathrm{k}} \otimes \omega_{1,1}, j=1, \ldots, s, \widetilde{\mathrm{F}}_{\mathrm{s}+1}=-\mathrm{I} \otimes \mathrm{L}_{1}$. With these normalizations we have

$$
\mathrm{sf}_{\mathrm{r}, \mathrm{s}+2}(\mathrm{~A}(\cdot))=\operatorname{ind}_{\mathrm{r}, \mathrm{s}+2}(\mathrm{D}) .
$$

Remark 8.2. There are other possible normalizations of course. For example, the roles of the $2 \times 2$ matrices $K_{1}, K_{2}$ can (up to sign) be reversed. The self-adjoint unitary $\frac{1}{\sqrt{2}}\left(K_{1}-K_{2}\right)$ conjugates $K_{1}$ and $-K_{2}$. It is then not hard to see that $D$ can be replaced by

$$
\mathrm{D}^{\prime}=\left(\begin{array}{cc}
\mathrm{A}(\mathrm{t}) & \frac{\mathrm{d}}{\mathrm{dt}} \\
\frac{\mathrm{d}}{\mathrm{dt}} & -\mathrm{A}(\mathrm{t})
\end{array}\right)=\mathrm{A}(\mathrm{t}) \otimes \mathrm{K}_{1}+\frac{\mathrm{d}}{\mathrm{dt}} \otimes \mathrm{K}_{2}
$$

with Clifford generators given by $\widetilde{E}_{j}:=E_{j} \otimes K_{1}, j=1, \ldots, r, \widetilde{F}_{k}:=F_{k} \otimes K_{1}, j=$ $1, \ldots, s, \widetilde{\mathrm{F}}_{s+1}=\mathrm{I} \otimes \mathrm{L}_{1}$.

Proof. For the analytic part of the theorem, the results of [RoSa95] and [KaLE13] apply. Note that we may write

$$
D=-\left(1 \otimes K_{1}\right)\left(\frac{d}{d t}-A(t) \otimes L_{1}\right) .
$$


Thus viewing $\mathrm{H} \otimes \mathbb{R}^{2}$ as a complex Hilbert space with complex structure $-\mathrm{I} \otimes \mathrm{L}_{1}$ we see that $\widetilde{A}(t):=-A(t) \otimes L_{1}$ is a family of self-adjoint Fredholm operators satisfying (A 1 )-(A 3).

Consequently, D is essentially skew-adjoint and Fredholm. Furthermore, a direct calculation shows that $\mathrm{D}$ is $\mathrm{C}_{\mathrm{r}, s+1}$-anti-linear with respect to the Clifford matrices $\widetilde{\mathrm{E}}_{1}, \ldots, \widetilde{\mathrm{E}}_{\mathrm{r}}, \widetilde{\mathrm{F}}_{1}, \ldots, \widetilde{\mathrm{F}}_{\mathrm{s}+1}$ given in the theorem.

It is clear that the right hand side of the claimed index formula satisfies homotopy invariance. Concatenation can either be proved directly by invoking classical methods of elliptic boundary value theory or as in [RoSA95, Prop. 4.26] where it is shown that concatenation already follows from homotopy and the easy to check constant and direct sums axioms.

It remains to check normalization which is done below.

8.3. Normalization. Let $\mathrm{V}$ be a finite dimensional $\mathrm{C}_{r, s+1}$-module and consider the path

$$
f(t) F_{s+1}=: A(t),
$$

where $f: \mathbb{R} \rightarrow \mathbb{R}$ is a continuous function with

$$
f(t)= \begin{cases}1, & t \leq 0 \\ -1, & t \geq 1\end{cases}
$$

The path $A(t)$ is homotopic to the straight line path from $F_{s+1}$ to $-F_{s+1}$ in the finite dimensional $C \ell_{r, s+1}$-module $V$, hence by Sec. 4.3 we have

$$
\operatorname{sf}_{r, s+2}(A(\cdot))=[V] \in A_{r, s+2} .
$$

We will need the $\mathrm{L}^{2}$-solutions of the ODE

$$
u^{\prime}+\varepsilon f u=0, \quad \varepsilon \in\{ \pm 1\}
$$

on $\mathbb{R}$. For $x \leq 0$ we have $u(x)=e^{-\varepsilon x} u(0)$ and for $x \geq 1$ we have $u(x)=e^{\varepsilon x} u(1)$. In both cases the solution is square integrable if and only if $\varepsilon=-1$. Thus, for $\varepsilon=1$ there are no $\mathrm{L}^{2}$-solutions and for $\varepsilon=-1$ there is a unique $\mathrm{L}^{2}$-solution $\bar{u}$ with $\bar{u}(0)=1$.

With regard to the consideration about $\mathrm{L}^{2}$-solutions we infer that the map

$$
\Phi: V \mapsto \operatorname{ker} D, \quad \eta \mapsto\left(x \mapsto \frac{\bar{u}(x)}{\sqrt{2}}\left(\begin{array}{l}
\eta-F_{s+1} \eta \\
\eta+F_{s+1} \eta
\end{array}\right)\right)
$$

is an isomorphism. One immediately checks, that $\Phi$ is $C \ell_{r, s+1}$-equivariant in the sense that $\Phi\left(E_{j} \eta\right)=\widetilde{E}_{j} \Phi(\eta), j=1, \ldots, r$ and $\Phi\left(F_{k} \eta\right)=\widetilde{F}_{k} \Phi(\eta), k=1, \ldots, s+1$. Thus $\Phi$ is an isomorphism of the Clifford module $\left(V ; E_{1}, \ldots, E_{r}, F_{1}, \ldots, F_{s+1}\right)$ onto the Clifford module $\left(\operatorname{ker} \mathrm{D} ; \widetilde{\mathrm{E}}_{1}, \ldots, \widetilde{\mathrm{E}}_{\mathrm{r}}, \widetilde{\mathrm{F}}_{1}, \ldots, \widetilde{\mathrm{F}}_{s+1}\right)$ and thus $\operatorname{ind}_{r, s+2} \mathrm{D}=[\mathrm{V}]=$ $\mathrm{sf}_{\mathrm{r}, \mathrm{s}+2}(A(\cdot))$. The proof of Theorem 8.1 is complete.

\section{Spectral flow and the Kasparov product}

In this section we relate Theorem 8.1 to Kasparov's theory via the unbounded Kasparov product. In order to have both a spectral flow and a Robbin-Salamon interpretation of the index theorems provided by Kasparov's theory, we must be 
in a setting where we have a one-parameter path of operators. For analogous results in the complex case, see [AzWA11, vDD19, KALE13].

We first note a word of caution in that up until this point, we have largely ignored the gradings on Clifford algebras. However, KK-theory is intrinsically $\mathbb{Z} / 2 \mathbb{Z}$-graded and as such all Clifford representations that appear as part of a Kasparov module must respect the grading. We will explicitly state when we can consider representations and modules as ungraded.

Throughout this section we use the identification of K-theory with KK-theory contained in the next Lemma.

Lemma 9.1. For $\mathcal{A}$ a $\sigma$-unital $\mathrm{C}^{*}$-algebra, we have for $\mathrm{s} \geq \mathrm{r}$

$$
\mathrm{KO}_{s-\mathrm{r}}(\mathcal{A}):=\operatorname{KKO}\left(\mathbb{R}, \mathcal{A} \otimes \mathrm{C}_{0}\left(\mathbb{R}^{s-r}\right)\right) \simeq \mathrm{KKO}\left(\mathrm{C}_{s, r}, \mathcal{A}\right)
$$

where the isomorphism is given by the Kasparov product with the class of the $(\mathbb{Z} / 2 \mathbb{Z}-$ graded) spectral triple

$$
\lambda_{s-r}=\left(C_{0}\left(\mathbb{R}^{s-r}\right) \otimes C \ell_{s-r, 0}, L^{2}\left(\mathbb{R}^{s-r}, \Lambda^{*} \mathbb{R}^{s-r}\right), d+d^{*}\right)
$$

with $\mathrm{d}+\mathrm{d}^{*}=\sum_{j=1}^{s-r} \partial_{x_{j}} \otimes \mathrm{f}_{j}$ using the isomorphism $\mathcal{B}\left(\Lambda^{*} \mathbb{R}^{\mathrm{k}}\right) \simeq \mathrm{C} \ell_{k, k}$.

Proof. This is a special case of [KAs80, Theorem 7, Sec. 5].

Let us first review the equivalence between paths of operators in $\mathcal{F}_{*}^{r, s}$ and Kasparov modules.

Proposition 9.2. Let $\{\mathrm{T}(\mathrm{t})\}_{\mathrm{t} \in \mathbb{R}}$ be a norm-continuous path in $\mathcal{F}_{*}^{\mathrm{r}, s+1}$ with $\mathrm{T}(\mathrm{t})$ invertible for all $\mathrm{t}$ outside the compact set $[-\mathrm{R}, \mathrm{R}]$. Then

$$
\left(\mathrm{C} \ell_{s+1, r},\left(\begin{array}{l}
\mathrm{H} \otimes \mathrm{C}_{0}(\mathbb{R}) \\
\mathrm{H} \otimes \mathrm{C}_{0}(\mathbb{R})
\end{array}\right)_{\mathrm{C}_{0}(\mathbb{R})},\left(\begin{array}{cc}
0 & -\mathrm{T}(\cdot) \\
\mathrm{T}(\cdot) & 0
\end{array}\right)\right)
$$

is a real Kasparov module where the left $\mathrm{Cl}_{\mathrm{s}+1, \mathrm{r}}$-representation is generated by the elements $\left\{\mathrm{I} \otimes \mathrm{K}_{2}, \mathrm{~F}_{1} \otimes \mathrm{L}_{1}, \ldots, \mathrm{F}_{\mathrm{s}} \otimes \mathrm{L}_{1}, \mathrm{E}_{1} \otimes \mathrm{L}_{1}, \ldots, \mathrm{E}_{\mathrm{r}} \otimes \mathrm{L}_{1}\right\}$.

Proof. Because $\{\mathbf{T}(\mathrm{t})\}_{\mathrm{t} \in \mathbb{R}}$ is a norm-continuous path of Fredholm operators invertible outside a compact set, it follows that $T(\cdot)$ is a well-defined operator on the $\mathrm{C}^{*}$-module $\mathrm{H} \otimes \mathrm{C}_{0}(\mathbb{R})$. It is then a simple check that the matrix $T(\cdot) \otimes \mathrm{L}_{1}$ is selfadjoint and anti-commutes (graded-commutes) with the left Clifford action.

Remark 9.3. Analogously to the previous proposition, if $A(\cdot): \mathbb{R} \mapsto \mathcal{F}^{\mathrm{r}, \mathrm{s}+1}(\mathrm{~W}, \mathrm{H})$ is a one-parameter family of skew-adjoint operators with compact resolvent and satisfying (A 1)-(A 3) as in the Robbin-Salamon theorem. Then we can construct an unbounded Kasparov module

$$
\left(C \ell_{s+1, r},\left(\begin{array}{l}
H \otimes C_{0}(\mathbb{R}) \\
H \otimes C_{0}(\mathbb{R})
\end{array}\right)_{C_{0}(\mathbb{R})},\left(\begin{array}{cc}
0 & -A(\cdot) \\
A(\cdot) & 0
\end{array}\right)\right)
$$

with Clifford generators as in Prop. 9.2. 
The properties of the spectral flow listed in Remark 5.2 ensure that $\mathrm{sf}_{\mathrm{r}, \mathrm{s}+2}$ descends to a map

$$
\mathrm{sf}_{\mathrm{r}, \mathrm{s}+2}: \mathrm{KO}_{\mathrm{s}+1-\mathrm{r}}\left(\mathrm{C}_{0}(\mathbb{R})\right) \rightarrow \mathrm{KO}_{s+2-r}(\mathbb{R}) .
$$

We will use Theorem 8.1 to compare spectral flow of the family $A(\cdot): \mathbb{R} \mapsto$ $\mathcal{F}^{\mathrm{r}, \mathrm{s}+1}(\mathrm{~W}, \mathrm{H})$ satisfying (A $\left.\mathrm{I}\right)-\left(\mathrm{A}_{3}\right)$ with the Kasparov product of the Kasparov module represented by Equation (9.2) with the fundamental class for $\mathbb{R}$. The fundamental class $\left[\partial_{x}\right] \in \mathrm{KKO}\left(\mathrm{C}_{0}(\mathbb{R}) \otimes C \ell_{1,0}, \mathbb{R}\right)$ of the real line is represented by the unbounded Kasparov module

$$
\left(C_{0}(\mathbb{R}) \otimes C \ell_{1,0}, L^{2}(\mathbb{R}) \otimes \Lambda^{*} \mathbb{R}, \partial_{x} \otimes f\right),
$$

where $\Lambda^{*} \mathbb{R} \simeq \mathbb{R}^{2}, f=\left(\begin{array}{cc}0 & -1 \\ 1 & 0\end{array}\right)$ and the representation of $C \ell_{1,0}$ is generated by $e=\left(\begin{array}{ll}0 & 1 \\ 1 & 0\end{array}\right)$

Proposition 9.4. Let $\mathrm{A}(\cdot): \mathbb{R} \mapsto \mathcal{F}^{\mathrm{r}, \mathrm{s}+1}(\mathrm{~W}, \mathrm{H})$ be a one-parameter family of skew-adjoint operators with compact resolvent and satisfying (A 1 )-(A 3). The Kasparov product $[A(\cdot)] \otimes_{\mathrm{C}_{0}(\mathbb{R})}\left[\partial_{x}\right]$ of the classes of the Kasparov modules (9.2) and (9.3) is represented by the Kasparov module

$$
\left(C l_{s+2, r}, L^{2}(\mathbb{R}, H) \otimes \Lambda^{*} \mathbb{R}^{2}, A(\cdot) \otimes f_{1}+\partial_{x} \otimes f_{2}\right)
$$

where we identify $\mathcal{B}\left(\Lambda^{*} \mathbb{R}^{2}\right) \simeq \mathrm{Cl}_{2,2}$ and the Clifford generators of the left action are $\left\{I \otimes e_{1}, I \otimes e_{2}, F_{1} \otimes f_{1}, \ldots, F_{s} \otimes f_{1}, E_{1} \otimes f_{1}, \ldots, E_{r} \otimes f_{1}\right\}$.

Applying the isomorphism $\phi: \mathrm{KKO}\left(\mathrm{Cl}_{s+2, r}, \mathbb{R}\right) \rightarrow \mathrm{KO}_{s+2-r}(\mathbb{R})$ to the product yields

$$
\phi\left([A(\cdot)] \otimes_{C_{0}(\mathbb{R})}\left[\partial_{x}\right]\right)=\operatorname{ind}_{r, s+2}\left(\begin{array}{cc}
A(\cdot) & \partial_{x} \\
\partial_{x} & -A(\cdot)
\end{array}\right)=-\operatorname{sf}_{r, s+2}(A(\cdot)) .
$$

Proof. To take the (unbounded) Kasparov product, we first take $d: C_{0}^{1}(\mathbb{R}) \rightarrow$ $\mathcal{B}\left(\mathrm{L}^{2}(\mathbb{R})\right)$ the trivial connection. Then making the identification

$$
\mathrm{C}_{0}(\mathbb{R}, \mathrm{H}) \otimes \mathrm{C}_{0}(\mathbb{R}) \mathrm{L}^{2}(\mathbb{R}) \rightarrow \mathrm{L}^{2}(\mathbb{R}, \mathrm{H}),
$$

we have that $1 \otimes_{d} \partial_{x}$ is represented as $\partial_{x}$ on $L^{2}(\mathbb{R}, H)$. We can similarly identify $\Lambda^{*} \mathbb{R} \otimes \Lambda^{*} \mathbb{R} \simeq \Lambda^{*} \mathbb{R}^{2}$ and so our product Hilbert space is $L^{2}(\mathbb{R}, H) \otimes \Lambda^{*} \mathbb{R}^{2}$. Putting together our Clifford actions, the results in [KALE13] ensure that the triple

$$
\left(C \ell_{s+2, r}, L^{2}(\mathbb{R}, H) \otimes \Lambda^{*} \mathbb{R}^{2}, A(\cdot) \otimes f_{1}+\partial_{x} \otimes f_{2}\right)
$$

is an unbounded representative of the product $[A(\cdot)] \otimes_{C_{0}(\mathbb{R})}\left[\partial_{x}\right]$.

We now relate our product spectral triple to a KO-theory class. We first identify $\Lambda^{*} \mathbb{R}^{2} \simeq \mathbb{R}^{4}$ and take the following generators of a $\mathrm{Cl}_{2,2}$-representation:

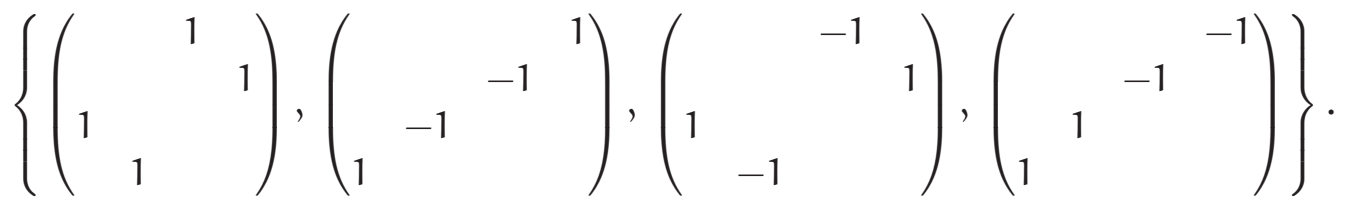


The product operator $T=A(\cdot) \otimes f_{1}+\partial_{x} \otimes f_{2}$ therefore has the form

$$
\mathrm{T}=\left(\begin{array}{cc}
\mathrm{O}_{2} & \mathrm{~T}_{+}^{*} \\
\mathrm{~T}_{+} & \mathrm{O}_{2}
\end{array}\right), \quad \mathrm{T}_{+}=\left(\begin{array}{cc}
\mathrm{A}(\cdot) & \partial_{\mathrm{x}} \\
\partial_{\mathrm{x}} & -\mathrm{A}(\cdot)
\end{array}\right) .
$$

We see that $T_{+}$is a skew-adjoint unbounded Fredholm operator on $L^{2}(\mathbb{R}, H)^{\oplus 2}$ anti-commuting with an ungraded left $\mathrm{C}_{r, s+1}$-action with generating elements $\left\{E_{1} \otimes K_{1}, \ldots, E_{r} \otimes K_{1}, F_{1} \otimes K_{1}, \ldots, F_{s} \otimes K_{1}, I \otimes L_{1}\right\}$. We can now apply the isomorphism $\phi: \mathrm{KKO}\left(\mathrm{Cl}_{s+2, r}, \mathbb{R}\right) \rightarrow \mathrm{KO}_{s+2-r}(\mathbb{R})$ which is given by

$$
\phi\left(\left[\left(\mathrm{Cl}_{s+2, r}, \mathrm{~L}^{2}(\mathbb{R}, \mathrm{H})^{\oplus 4}, \mathrm{~T}\right)\right]\right)=\operatorname{ind}_{r, s+2}\left(\mathrm{~T}_{+}\right) \in \mathrm{A}_{\mathrm{r}, \mathrm{s}+2} \simeq \mathrm{KO}_{s+2-\mathrm{r}}(\mathbb{R}) .
$$

Therefore, we find that

$$
\phi\left([A(\cdot)] \otimes_{C_{0}(\mathbb{R})}\left[\partial_{x}\right]\right)=\operatorname{ind}_{r, s+2}\left(\begin{array}{cc}
A(\cdot) & \partial_{x} \\
\partial_{x} & -A(\cdot)
\end{array}\right)=-\operatorname{sf}_{r, s+2}(A(\cdot)) .
$$

The last equality comes from Theorem 8.1 and by conjugating $T_{+}$by the selfadjoint unitary $\frac{1}{\sqrt{2}}\left(K_{1}-K_{2}\right)$, cf. Remark 8.2, which will reverse the orientation of $\mathrm{Cl}_{2,0}$ and sends the class in $\mathrm{KO}_{s+2-r}(\mathbb{R})$ to its inverse.

The minus sign relating the Kasparov product to the $\mathrm{KO}$-valued spectral flow is common in such index formulas. In the case that $r=s$ and $r-s=1, S F_{r, s+2}$ has range $\mathbb{Z} / 2 \mathbb{Z}$ and so this minus sign can be ignored.

\section{APPENDix A. SOME CLASSICAL HOMOTOPY EQUIVALENCES}

In this appendix we will explicitly describe a few useful homotopy equivalences most of which are reformulations of results due to Atiyah and Singer [AtSi69]. We will use freely the notation introduced in Sec. 2.1.

Strictly speaking, [AтSI69] cover "only" the case of $\mathrm{Cl}_{0, k}$ symmetry. Note, however, that due to the isomorphisms Eq. (2.6)-Eq. (2.9) one has the following: if $X^{r, s}$ denotes any of the spaces $\mathcal{F}^{\mathrm{r}, s+1}, \widetilde{\Omega}_{r, s}, \ldots$ etc. then $X^{\mathrm{r}+1, s+1}$ is naturally homeomorphic to $X^{r, s}$. Also, in a standard Hilbert space, $X^{r+8, s}, X^{r, s+8}$ are homeomorphic to $\mathrm{X}^{\mathrm{r}, \mathrm{s}}$ and the homeomorphism is compatible with all the homotopy equivalences stated below. ${ }^{11}$ Therefore, we just summarize the results. The easy (but tedious) details are left to the reader.

\section{A.1. Homotopy results for standard $\mathrm{C}_{r, s}-$ Hilbert spaces.}

Proposition A.1. For a $C \ell_{r, s}-$ Hilbert space $\mathrm{H}$ the inclusion $\bar{\Omega}_{\mathrm{r}, \mathrm{s}} \subset \widetilde{\Omega}_{\mathrm{r}, \mathrm{s}}$ is a weak homotopy equivalence. ${ }^{12}$

Proof. As in [ATSi69] one concludes that $\bar{\Omega}_{r, s} \hookrightarrow \widetilde{\Omega}_{r, s} \rightarrow\left\{x \in \mathcal{J}^{r, s}(Q(H)) \mid \| x-\right.$ $\left.\pi\left(F_{s}\right) \|_{2}<2\right\}$ is a fibration. In view of Prop. 3.2 the base is contractible, whence the claim.

\footnotetext{
${ }^{11}$ This is a metastatement whose details are hard to formulate such that a lawyer will be happy; however every literate reader will be able to fill in the correct details.

${ }^{12} \mathrm{~A}$ weak or singular homotopy equivalence is a map which induces isomorphisms on all homotopy groups
} 
Next we recall the main results of [ATSi69]:

Theorem A.2 ([AtSi69], Theorems $\mathrm{A}(\mathrm{k}), \mathrm{B}(\mathrm{k}))$. Let $\mathrm{H}$ be a standard $\mathrm{C}_{\mathrm{r}, \mathrm{s}}-$ Hilbert space. Then

$$
\operatorname{ind}_{r, s+1}: \mathcal{F}_{*}^{r, s+1} \rightarrow A_{r, s+1}=\mathrm{KO}_{s+1-r}(\mathbb{R}), \quad \mathrm{T} \mapsto[\operatorname{ker} T]_{\mathcal{M}_{r, s} / \mathcal{M}_{r, s+1}}
$$

labels $\pi_{0}\left(\mathcal{F}_{*}^{r, s+1}\right)$.

This means that each $T \in \mathcal{F}_{*}^{r, s+1}$ can be path connected to an operator $T_{V}$ of the normal form Eq. (2.24).

Theorem A.3 ([ATSi69], Theorems A(k), B(k)). Let $s>0$ or $r=s=0$. Then for a standard $\mathrm{C}_{\mathrm{r}, \mathrm{s}}-$ Hilbert space, the map

$$
\begin{aligned}
& \mathrm{FO}_{*}^{\mathrm{r}, \mathrm{s}+1} \rightarrow \Omega\left(\mathrm{FO}_{*}^{\mathrm{r}, \mathrm{s}}\right), \quad \mathrm{T} \mapsto \alpha_{\bullet}(\mathrm{T}), \\
& \alpha_{\mathrm{t}}(\mathrm{T}):=\cos (\pi \mathrm{t}) \mathrm{F}_{\mathrm{s}}+\sin (\pi \mathrm{t}) \mathrm{T}, \quad 0 \leq \mathrm{t} \leq 1
\end{aligned}
$$

is a homotopy equivalence. The RHS is the space of paths from $\mathrm{F}_{\mathrm{s}}$ to $-\mathrm{F}_{\mathrm{s}}$ in $\mathrm{FO}_{*}^{\mathrm{r}, \mathrm{s}}$.

In the corner case $\mathrm{r}=\mathrm{s}=0$ this must be read as follows: $\mathrm{FO}_{*}^{0,0}$ is the space of essentially unitary Fredholm operators and $\mathrm{F}_{0}=\mathrm{I}$.

Theorem A.4. Let $\mathrm{s}>0$ or $\mathrm{r}=\mathrm{s}=0$ and let $\mathrm{H}$ be a standard $\mathrm{C} \ell_{\mathrm{r}, \mathrm{s}}$-Hilbert space. Then the map

$$
\Phi_{\mathrm{r}, \mathrm{s}+1}: \mathrm{FO}_{*}^{\mathrm{r}, \mathrm{s}+1} \rightarrow \bar{\Omega}_{\mathrm{r}, \mathrm{s}}, \quad \mathrm{T} \mapsto-\mathrm{F}_{\mathrm{s}} \mathrm{e}^{\pi \mathrm{TF} \mathrm{F}_{\mathrm{s}}}
$$

is a homotopy equivalence. Again, if $\mathrm{r}=\mathrm{s}=0$ then $\mathrm{F}_{0}=\mathrm{I}$. Putting

$$
\Psi: i \mathbb{R} \rightarrow i \mathbb{R}, \quad x \mapsto \begin{cases}x, & |x| \leq 1, \\ x /|x|, & |x| \geq 1,\end{cases}
$$

one obtains a homotopy equivalence

$$
\Phi_{\Psi}: \mathcal{F}_{*}^{\mathrm{r}, \mathrm{s}+1} \rightarrow \widetilde{\Omega}_{\mathrm{r}, \mathrm{s}}, \quad \mathrm{T} \mapsto-\mathrm{F}_{\mathrm{s}} \mathrm{e}^{\pi \Psi\left(\mathrm{TF}_{\mathrm{s}}\right)} .
$$

Proof. The claim about the first homotopy equivalence follows from [AtSi69], Prop. $4.2(s>0)$ and Prop. $3.3(r=s=0)$. The second claim is then a simple consequence since on the deformation retract $\mathrm{FO}_{*}^{\mathrm{r}, s+1} \subset \mathcal{F}_{*}^{\mathrm{r}, s+1}$ the map $\Phi_{\Psi}$ coincides with the map $\Phi_{r, s+1}$.

We note that $\Phi_{\Psi}\left( \pm \mathrm{F}_{s+1}\right)=\Phi_{r, s+1}\left( \pm \mathrm{F}_{s+1}\right)=\mathrm{F}_{\mathrm{s}}$ so that $\Phi_{\Psi}, \Phi_{\mathrm{r}, \mathrm{s}+1}$ sends paths from $F_{s+1}$ to $-F_{s+1}$ in $\mathcal{F}_{*}^{r, s+1}, F_{*}^{r, s+1}$ to loops in $\bar{\Omega}_{r, s}$ with base point $F_{s}$. As a corollary we obtain the characterization of the connected components of $\widetilde{\Omega}^{r, s+1}$ :

Corollary A.5. Let $\mathrm{s}>0$ or $\mathrm{r}=\mathrm{s}=0$ and let $\mathrm{H}$ be a standard $\mathrm{C}_{\mathrm{r}, \mathrm{s}}$-Hilbert space. Then we have canonical identifications $\pi_{0}\left(\widetilde{\Omega}_{\mathrm{r}, \mathrm{s}}\right) \simeq \pi_{0}\left(\bar{\Omega}_{\mathrm{r}, \mathrm{s}}\right) \simeq \mathrm{A}_{\mathrm{r}, \mathrm{s}+1} \simeq \mathrm{KO}_{\mathrm{s}+1-\mathrm{r}}(\mathbb{R})$. More concretely, each path component contains an element which, w.r.t. a $\mathrm{Cl}_{\mathrm{r}, \mathrm{s}}$-linear decomposition $\mathrm{H}=\mathrm{H}_{0} \oplus \mathrm{V}$, takes the form $\left.\mathrm{F}_{\mathrm{s}}\right|_{\mathrm{H}_{0}} \oplus-\left.\mathrm{F}_{\mathrm{s}}\right|_{\mathrm{V}}$. Under the claimed isomorphism this element is then mapped to $[\mathrm{V}] \in \mathcal{M}_{\mathrm{r}, \mathrm{s}} / \mathcal{M}_{\mathrm{r}, \mathrm{s}+1}$. 
Proof. The first isomorphism is Prop. A.1. According to the remark after Theorem A.2 each class in $\pi_{0}\left(\mathcal{F}_{*}^{r, s+1}\right)$ has a representative $T=F_{s+1} \oplus 0$ w.r.t. the $\mathrm{Cl}_{r, s}$-linear decomposition $\mathrm{H}=\mathrm{H}_{0} \oplus \mathrm{V}$ such that $\mathrm{H}_{0}$ carries a $\mathrm{C}_{r, s+1}$ structure; then $\operatorname{ind}_{r, s+1}(T)=[V]$. Under the homotopy equivalence $\Phi_{r, s+1}$ of Theorem A.4 this is mapped to

$$
\Phi_{r, s+1}(T)=-\left.F_{s+1} e^{\pi F_{s+1} F_{s}}\right|_{H_{0}} \oplus-\left.F_{s}\right|_{V}=\left.F_{s}\right|_{H_{0}} \oplus-\left.F_{s}\right|_{V},
$$

since $\left(F_{s+1} F_{s}\right)^{2}=-I$.

Now we are in the position to prove the main result of this section:

Theorem A.6. Let $\mathrm{s}>0$ or $\mathrm{r}=\mathrm{s}=0$ and let $\mathrm{H}$ be a standard $\mathrm{C} \ell_{r, s+1}$-Hilbert space. Then the fundamental group $\pi_{1}\left(\widetilde{\Omega}_{r, s}, F_{s}\right)$ is canonically isomorphic to $\mathcal{M}_{r, s+1} / \mathcal{M}_{r, s+2} \simeq$ $\mathrm{KO}_{s+2-r}(\mathbb{R})$.

The isomorphism is given as follows: given a loop $\gamma:[0,1] \rightarrow \widetilde{\Omega}_{r, s}, \gamma(0)=\gamma(1)=F_{s}$, then there is a $\mathrm{Cl}_{r, s+1}$ decomposition $\mathrm{H}=\mathrm{H}_{0} \oplus \mathrm{V}$ with $\operatorname{dim} \mathrm{V}<\infty$ and $\mathrm{H}_{0}$ carrying a $\mathrm{C}_{r, s+2}$-structure such that $\gamma$ is homotopic to the loop

$$
\begin{aligned}
\widetilde{\gamma}(\mathrm{t}) & :=\left.\left.\mathrm{F}_{\mathrm{s}}\right|_{\mathrm{H}_{0}} \oplus \mathrm{F}_{\mathrm{s}} \mathrm{e}^{-2 \pi \mathrm{t} \mathrm{F}_{\mathrm{s}+1} \mathrm{~F}_{\mathrm{s}}}\right|_{\mathrm{V}} \\
& =\left.\left.\mathrm{F}_{\mathrm{s}}\right|_{\mathrm{H}_{0}} \oplus\left(\cos (2 \pi \mathrm{t}) \mathrm{F}_{\mathrm{s}}-\sin (2 \pi \mathrm{t}) \mathrm{F}_{\mathrm{s}+1}\right)\right|_{\mathrm{V}}, \quad 0 \leq \mathrm{t} \leq 1 .
\end{aligned}
$$

The element $[\gamma]$ is then mapped to $[\mathrm{V}] \in \mathcal{M}_{\mathrm{r}, \mathrm{s}+1} / \mathcal{M}_{\mathrm{r}, \mathrm{s}+2}$.

As usual, in the case $\mathrm{r}=\mathrm{s}=0$ one has to read the formulas with $\mathrm{F}_{0}=\mathrm{I}$.

Proof. In view of Prop. A.I it suffices to prove the result with $\bar{\Omega}_{r, s}$ instead of $\widetilde{\Omega}_{r, s}$.

Pick a typical representative T of $\mathcal{M}_{r, s+1} / \mathcal{M}_{r, s+2}$ as in the proof of Cor. A.5. According to the isomorphism in Theorem A.2 this labels $\pi_{0}\left(\mathrm{FO}_{*}^{\mathrm{r}, s+2}\right)$. Under the isomorphism in Theorem A.3, $\pi_{0}\left(\mathrm{FO}_{*}^{\mathrm{r}, s+2}\right)$ is isomorphic to the homotopy classes of paths in $\mathrm{FO}_{*}^{\mathrm{r}, \mathrm{s}+1}$ from $\mathrm{F}_{\mathrm{s}+1}$ to $-\mathrm{F}_{\mathrm{s}+1}$. For $\mathrm{T}=\mathrm{F}_{\mathrm{s}+2} \oplus 0, \mathrm{H}=\mathrm{H}_{0} \oplus \mathrm{V}$ we first compute $\alpha_{t}(T)$ of Theorem A.3: on $\mathrm{H}_{0}$ we have $\alpha_{\mathrm{t}}\left(\mathrm{F}_{s+2}\right)=\cos (\pi \mathrm{t}) \mathrm{F}_{s+1}+\sin (\pi \mathrm{t}) \mathrm{F}_{s+2}$ while on $V$ we have $\alpha_{t}(0)=\cos (\pi t) F_{s+1}$.

Finally, the map $\Phi_{r, s+1}$ of Theorem A.4 maps the homotopy classes of paths in $\mathrm{FO}_{*}^{\mathrm{r}, s+1}$ from $\mathrm{F}_{s+1}$ to $-\mathrm{F}_{s+1}$ to $\pi_{1}\left(\bar{\Omega}_{\mathrm{r}, s}, \mathrm{~F}_{\mathrm{s}}\right)$. On $\mathrm{H}_{0}$ we have $\Phi_{\mathrm{r}, \mathrm{s}+1}\left(\alpha_{\mathrm{t}}\left(\mathrm{F}_{\mathrm{s}+2}\right)\right)=$ $-\mathrm{F}_{\mathrm{s}} \mathrm{e}^{\pi \alpha_{\mathrm{t}}\left(\mathrm{F}_{\mathrm{s}+2} \mathrm{~F}_{\mathrm{s}}\right)} \equiv \mathrm{F}_{\mathrm{s}}$ since $\alpha_{\mathrm{t}}\left(\mathrm{F}_{\mathrm{s}+1}\right)$ anti-commutes with $\mathrm{F}_{\mathrm{s}}$, is skew and has square -1 .

On $V$ we have $\Phi_{r, s+1}\left(\alpha_{t}(0)\right)=-F_{s} e^{\pi \cos (\pi t) F_{s+1} F_{s}}, 0 \leq t \leq 1$. As $\cos (\pi t)$ runs from 1 to -1 this path is homotopic to $-F_{s} e^{\pi(1-2 t) F_{s+1} F_{s}}=F_{s} e^{-2 \pi t F_{s+1} F_{s}}, 0 \leq t \leq 1$, completing the proof.

A.2. Stable homotopy in the finite dimensional case. Up to this point the results of this Appendix A are proved by finite dimensional approximation. This means that they do have finite dimensional analogues. Here, the infinite multiplicity assumption built-in to the notion of a standard $\mathrm{C}_{r, s}-$ Hilbert space needs to be replaced by looking at stable homotopy groups.

We will need the stable analogues of Cor. A.5 and Theorem A.6. The statements can be extracted from [ATSi69]. However, they can also be found in Milnor's book [MiL63, Sec. 24]. 
For a finite dimensional $C \ell_{r, s}-$ module $V$ one has $\bar{\Omega}_{r, s}(V)=\widetilde{\Omega}_{r, s}(V)=: \Omega_{r, s}(V)$. If $V \hookrightarrow W$ is an inclusion of finite dimensional $\mathrm{C}_{r, s}$-modules then there is an obvious inclusion $\Omega_{r, s}(\mathrm{~V}) \rightarrow \Omega_{r, s}(\mathrm{~W})$ by sending $\left.\mathrm{J} \mapsto \mathrm{J} \oplus \mathrm{F}_{\mathrm{s}}\right|_{\mathrm{V}_{\perp}}$. Maps $\mathrm{f}, \mathrm{g}: \mathrm{X} \rightarrow$ $\Omega_{r, s}(V)$ are said to be stably homotopic if they become homotopic after embedding into $\Omega_{r, s}(W)$ for a sufficiently high dimensional $C \ell_{r, s}-$ module $W \supset V$. In this way one obtains stable homotopy sets/groups $\pi_{0}^{\mathrm{S}}\left(\Omega_{r, s}(\mathrm{~V})\right), \pi_{1}^{\mathrm{S}}\left(\Omega_{r, s}(\mathrm{~V})\right)$.

Theorem A.7. Let $\mathrm{H}$ be a finite dimensional $\mathrm{C}_{\mathrm{r}, \mathrm{s}}-$ module. Then the stable homotopy set $\pi_{0}^{S}\left(\Omega_{r, s}(V)\right)$ is canonically isomorphic to $\mathcal{M}_{r, s} / \mathcal{M}_{r, s+1}$. The concrete generators are the same as those mentioned in Cor. A.5.

Similarly, for a finite dimensional $\mathrm{C}_{\mathrm{r}, \mathrm{s}+1}-$ module $\mathrm{H}$, the stable fundamental group $\pi_{1}^{\mathrm{S}}\left(\Omega_{r, s}(\mathrm{H}), \mathrm{F}_{\mathrm{s}}\right)$ is canonically isomorphic to $\mathcal{M}_{r, s+1} / \mathcal{M}_{r, s+2}$. The concrete generators are the same as those mentioned in Theorem A.6.

\section{REFERENCES}

[ABS64] M. F. АтіYAн, R. Bотт, and A. Shapiro, Clifford modules, Topology 3 (1964), no. suppl. 1, 3-38. MR 0167985

[AlZig7] A. Altland and M. R. Zirnbauer, Nonstandard symmetry classes in mesoscopic normal/superconducting hybrid structures, Phys. Rev. B 55 (1997), 1142-1162.

[AMZ19] A. Alldridge, C. Max, and M. R. Zirnbauer, Bulk-boundary correspondence for disordered free-fermion topological phases, Comm. Math. Phys., online first (2019). https:dx.doi.org/10.1007/s00220-019-03581-7

[APS76] M. F. Atiyah, V. K. Patodi, and I. M. Singer, Spectral asymmetry and Riemannian geometry. III, Math. Proc. Cambridge Philos. Soc. 79 (1976), no. 1, 71-99. MR 0397799 (53 \#1655c)

[ASS94A] J. Avron, R. SEILER, and B. Simon, The index of a pair of projections, J. Funct. Anal. 120 (1994), no. 1, 220-237. MR 1262254 (95b:47012)

[ASS94B] J. Avron, R. Seiler, and B. Simon, Charge deficiency, charge transport and comparison of dimensions, Comm. Math. Phys. 159 (1994), 399-422. MR 1256994

[Aті66] M. F. Aтіүaн, K-theory and reality, Quart. J. Math. Oxford Ser. (2) 17 (1966), 367-386. MR 0206940

[AtSi69] M. F. Аtiyah and I. M. Singer, Index theory for skew-adjoint Fredholm operators, Inst. Hautes Études Sci. Publ. Math. (1969), no. 37, 5-26. MR 0285033

[AzWa11] S. AzzAli and C. WAHL, Spectral flow, index and the signature operator, J. Topol. Anal. 3 (2011), no. 1, 37-67. MR 2784763

[Bla98] B. Blackadar, K-theory for operator algebras, second ed., Mathematical Sciences Research Institute Publications, vol. 5, Cambridge University Press, Cambridge, 1998. MR 1656031 (99g:46104)

[BBLPo5] B. Booss-BAvnbeK, M. Lesch, and J. Phillips, Unbounded Fredholm operators and spectral flow, Canad. J. Math. 57 (2005), no. 2, 225-250. MR 2124916

[BBWo93] B. Booss-BAvnbeK and K. P. WojciechowsKi, Elliptic boundary problems for Dirac operators, Mathematics: Theory \& Applications, Birkhäuser Boston Inc., Boston, MA, 1993. MR 1233386 (94h:58168)

[BCRi6] C. Bourne, A. L. Carey, and A. Rennie, A non-commutative framework for topological insulators, Rev. Math. Phys. 28 (2016), no. 2, 1650004, 51. MR 3484317

[BKR17] C. Bourne, J. KellendonK, and A. Rennie, The K-theoretic bulk-edge correspondence for topological insulators, Ann. Henri Poincaré 18 (2017), no. 5, 1833-1866. MR 3635969

[BoSB2o] C. Bourne and H. Schulz-BAldes, On $\mathbb{Z}_{2}$-indices for ground states of fermionic chains, Rev. Math. Phys. 32 (2020), 2050028. 
[BDF73] L. G. Brown, R. G. Douglas, and P. A. Fillmore, Unitary equivalence modulo the compact operators and extensions of $\mathrm{C}^{*}$-algebras, 58-128. Lecture Notes in Math., Vol. 345. MR 0380478

[BRLEOI] J. BRÜNING and M. LESCH, On boundary value problems for Dirac type operators. I. Regularity and self-adjointness, J. Funct. Anal. 185 (2001), no. 1, 1-62. arXiv: 9905181 [math.FA], MR 1853751 (2002g:58034)

[CPSB19] A. L. Carey, J. Phillips, and H. Schulz-Baldes, Spectral flow for skew-adjoint Fredholm operators, J. Spectr. Theory 9 (2019), no. 1, 137-170. MR 3900782

[CASB19] A. L. Carey and H. Schulz-Baldes, Spectral flow of monopole insertion in topological insulators, Comm. Math. Phys., 370 (2019), no. 3, 895-923. MR 3995923

[DNSB16] G. De Nittis and H. Schulz-Baldes, Spectral flows associated to flux tubes, Ann. Henri Poincaré, 17 (2016), 1-35. MR 3437823

[DSBW19] N. Doll, H. Schulz-Baldes, and N. Waterstraat, Parity as $\mathbb{Z}_{2}$-valued spectral flow, Bull. London Math. Soc., 51 (2019), 836-852 (2019). MR 4022430

[vDDi9] K. van DEN Dungen, The index of generalised Dirac-Schrödinger operators, J. Spectr. Theory 9 (2019), no. 4, 1459-1506. MR 4033528

[FrMo13] D. S. Freed and G. W. Moore, Twisted equivariant matter, Ann. Henri Poincaré 14 (2013), no. 8, 1927-2023. MR 3119923

[GrSB16] J. Grossmann and H. Schulz-BALDEs, Index pairings in presence of symmetries with applications to topological insulators, Comm. Math. Phys. 343 (2016), no. 2, 477-513. MR 3477345

[HHZo5] P. Heinzner, A. Huckleberry, and M. R. Zirnbauer, Symmetry classes of disordered fermions, Comm. Math. Phys. 257 (2005), no. 3, 725-771. MR 2164950

[KaLe13] J. KAAD and M. Lesch, Spectral flow and the unbounded Kasparov product, Adv. Math. 248 (2013), 495-530. arXiv:1110.1472 [math.OA], MR 3107519

[Kar7o] M. Karoubi, Espaces classifiants en K-théorie, Trans. Amer. Math. Soc. 147 (1970), 75-115. MR 0261592

[Kas8o] G. G. Kasparov, The operator K-functor and extensions of $\mathrm{C}^{*}$-algebras, Izv. Akad. Nauk SSSR Ser. Mat. 44 (1980), no. 3, 571-636, 719. MR 582160

[Kat95] T. Kato, Perturbation theory for linear operators, Classics in Mathematics, Springer-Verlag, Berlin, 1995, Reprint of the 1980 edition. MR 1335452 (96a:47025)

[KaKo18] H. Katsura and T. Koma, The noncommutative index theorem and the periodic table for disordered topological insulators and superconductors, J. Math. Phys. 59 (2018) 031903. MR 3774825

[KeL17] J. Kellendonk, On the $\mathrm{C}^{*}$-algebraic approach to topological phases for insulators, Ann. Henri Poincaré 18 (2017), no. 7, 2251-2300. MR 3665214

[KeL19] Cyclic cohomology for graded $\mathrm{C}^{*, \mathrm{r}}$-algebras and its pairings with van Daele K-theory, Comm. Math. Phys. 368 (2019), no. 2, 467-518. MR 3949717

[KeZI16] R. KenNedy and M. R. ZirnBAuER, Bott periodicity for $\mathbb{Z}_{2}$ symmetric ground states of gapped free-fermion systems, Comm. Math. Phys. 342 (2016), no. 3, 909-963. MR 3465435

[KILEO4] P. KIRK and M. Lesch, The $\eta$-invariant, Maslov index, and spectral flow for Dirac-type operators on manifolds with boundary, Forum Math. 16 (2004), no. 4, 553-629. arXiv: 0012123 [math.DG], MR 2044028 (2005b:58029)

[Kito9] A. Kitaev, Periodic table for topological insulators and superconductors, Proceedings of the L.D.Landau Memorial Conference 'Advances in Theoretical Physics' (V. Lebedev and M. Feigel'man, eds.), vol. 1134, American Institute of Physics Conference Series, 2009, pp. 22-30.

[LaMi89] H. B. Lawson, JR. and M.-L. Michelsohn, Spin geometry, Princeton Mathematical Series, vol. 38, Princeton University Press, Princeton, NJ, 1989. MR 1031992

[Leso5] M. Lesch, The uniqueness of the spectral flow on spaces of unbounded self-adjoint Fredholm operators, Spectral geometry of manifolds with boundary and decomposition of manifolds, Contemp. Math., vol. 366, Amer. Math. Soc., Providence, RI, 2005, pp. 193-224. MR 2114489 
[LiMo19] Z. LI and R. Mong Local formula for the $\mathbb{Z}_{2}$-invariant of topological insulators, Phys. Rev. B 100 (2019) 205101.

[Lot88] J. Lotr, Real anomalies, J. Math. Phys. 29 (1988), no. 6, 1455-1464. MR 944463

[Mil63] J. Milnor, Morse theory, Based on lecture notes by M. Spivak and R. Wells. Annals of Mathematics Studies, No. 51, Princeton University Press, Princeton, N.J., 1963. MR о163331

[Phig6] J. Phillips, Self-adjoint Fredholm operators and spectral flow, Canad. Math. Bull. 39 (1996), no. 4, 460-467. MR 1426691

[RoSa95] J. Robbin and D. Salamon, The spectral flow and the Maslov index, Bull. London Math. Soc. 27 (1995), no. 1, 1-33. MR 1331677 (96d:58021)

[RSFlio] S. Ryu, A. P. Schnyder, A. Furusaki and A. W. W. Ludwig, Topological insulators and superconductors: ten-fold way and dimensional hierarchy, New J. Phys. 12 (2010), 065010.

[SсH93] H. SchröDER, K-theory for real $\mathrm{C}^{*}$-algebras and applications, Pitman Research Notes in Mathematics Series, vol. 290, Longman Scientific \& Technical, Harlow; copublished in the United States with John Wiley \& Sons, Inc., New York, 1993. MR 1267059

[Thi16] G. C. Thiang, On the K-theoretic classification of topological phases of matter, Ann. Henri Poincaré 17 (2016), no. 4, 757-794. MR 3472623

[Wit82] E. WitTen, An SU(2) anomaly, Phys. Lett. B 117 (1982), no. 5, 324-328. MR 678541

WPI-Advanced Institute for Materials Research (WPI-AIMR), TOHOKu UNIVERsity, 2-1-1 Katahira, Аовa-KU, Sendai, 980-8577, Japan ANd RIKEN iTHEMS, Wako, Saitama 351-0198, JAPAN

E-mail address: chris.bourne@tohoku.ac.jp

Mathematical Sciences Institute, Australian National University, Kingsley St., Canberra, ACT 0200, Australia and School of Mathematics and Applied Statistics, University of Wollongong, NSW, Australia, 2522

E-mail address: acareyemaths. anu . edu.au

URL: http://maths.anu.edu.au/ acarey/

Mathematisches Institut, Universität Bonn, Endenicher Allee 6o, 53115 Bonn, Germany

E-mail address: ml@matthiaslesch.de, lesch@math.uni-bonn.de

URL: www.matthiaslesch.de, www.math.uni-bonn.de/people/lesch

School of Mathematics and Applied Statistics, University of Wollongong, NSW, AusTRALIA, 2522

E-mail address: renniea@uow.edu.au 